\title{
Salicylic Acid (SA)-independent processes in Systemic Acquired Resistance (SAR)
}

\author{
Dissertation \\ for the award of the degree \\ "Doctor rerum naturalium" \\ of the Georg-August-University Göttingen
}

within the doctoral program IRTG 2172 PRoTECT

of the Georg-August University School of Science (GAUSS)

submitted by

Aswin Nair

From Calicut, India

Göttingen, 2021 


\section{Thesis Committee}

Prof. Dr. Christiane Gatz, Plant Molecular Biology and Physiology, Albrecht-von-HallerInstitute for Plant Sciences, Georg-August-University Göttingen

Prof. Dr. Ivo Feußner, Plant Biochemistry, Albrecht-von-Haller-Institute for Plant Sciences, Georg-August-University Göttingen

Dr. Yuelin Zhang, Department of Botany, University of British Columbia, Vancouver, Canada

\section{Members of the Examination Board}

Referee

Prof. Dr. Christiane Gatz, Plant Molecular Biology and Physiology, Albrecht-von-HallerInstitute for Plant Sciences, Georg-August-University Göttingen

$2^{\text {nd }}$ Referee

Prof. Dr. Ivo Feußner, Plant Biochemistry, Albrecht-von-Haller-Institute for Plant Sciences, Georg-August-University Göttingen

\section{Further members of the Examination Board}

Prof. Dr. Andrea Polle, Forest Botany and Tree Physiology, Büsgen-Institute, GeorgAugust University of Göttingen

Prof. Dr. Kai Heimel, Molecular Microbiology and Genetics, Georg-August-University Göttingen

PD Dr. Marcel Wiermer, Plant Cell Biology - Molecular Biology of Plant-Microbe Interactions, Albrecht-von-Haller-Institute, Georg-August-University Göttingen

PD Dr. Till Ischebeck, Plant Biochemistry, Albrecht-von-Haller-Institute for Plant Sciences, Georg-August-University Göttingen

Date of oral examination: 27/03/2020 



\section{Contents}

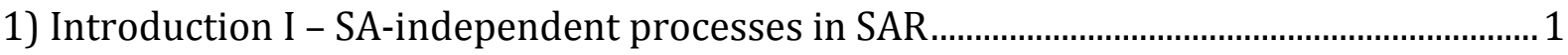

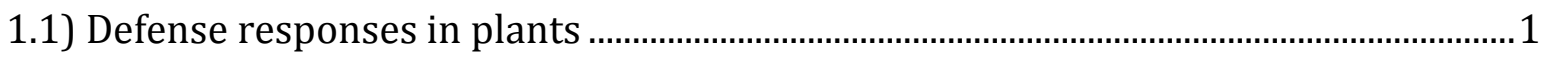

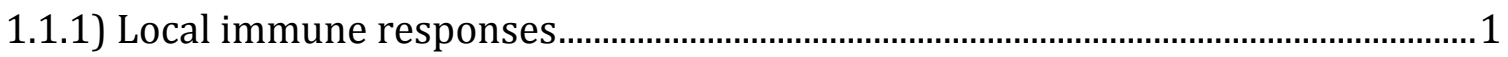

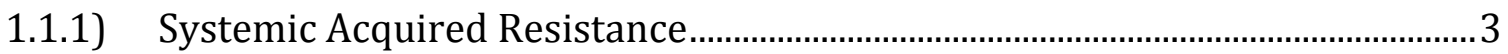

1.2) SA as a critical player in the establishment of SAR .....................................................

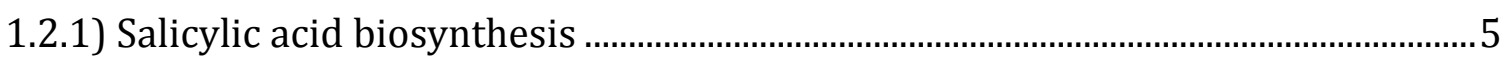

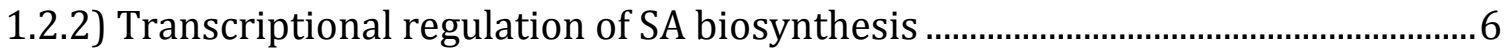

1.2.3) Perception of SA by NON EXPRESSOR OF PATHOGENESIS-RELATED GENES 1

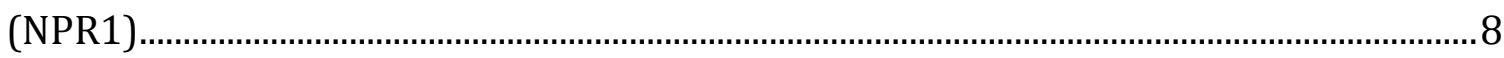

1.2.4) Class I and II TGA transcription factors as mediators of NPR1 function during SAR .

1.3) Pipecolic acid and N-hydroxy pipecolic acid (NHP) - new players in the field ...... 11

1.3.1) Pipecolic acid and NHP biosynthesis..................................................................... 11

1.3.2) Transcriptional regulation of NHP biosynthesis and downstream signaling.. 13

1.4) ENHANCED DISEASE SUSCEPTIBILITY 1 (EDS1) as an essential component of the transcriptional reprogramming during SAR 14

1.5) Interconnection between SA and NHP synthesis 15

2) Introduction II - The glutaredoxin ROXY19 is a negative regulator of the JA/ETdependent defense pathway 18

2.1) Glutaredoxins (GRXs) in Arabidopsis. 18

2.1.1) Class I GRXs in plants. 20

2.1.2) Class II GRXs in plants. 21 
2.2) Regulation of developmental processes by ROXY1 and the TGA transcription factor PERIANTHIA (PAN)

2.3) Regulation of immune-related genes by ROXY18 and ROXY19. 23

3) Aim of this thesis. 26

4) Materials 27

4.1) Organisms 27

4.2) Primers 28

4.3) Plasmids 28

4.4) Antibodies. 29

4.5) Chemicals 29

4.6) Kits and enzymes 31

5) Methods 48

5.1) Work with organisms 48

5.1.1) Arabidopsis thaliana surface sterilization and growth conditions 48

5.1.2) Systemic Acquired Resistance (SAR) experiments. 48

5.1.3) Cultivation of Pseudomonas syringae pv. maculicola ES4326 (Psm) 49

5.1.4) Bacterial growth assay. 50

5.1.5) Pharmacological treatment with SA or NHP 50

5.1.6) Botrytis cinerea infection assays 51

5.1.7) Cultivation of Escherichia coli. 51

5.1.8) Recombination in DH10 $\alpha$ BacY (Protocol from Cramer Lab - MPI Biophysical Chemistry, Göttingen) 
5.1.9) Isolation of bacmid DNA by alkaline lysis/ isopropanol /ethanol precipitation

5.1.10) Insect cell lines used for bacmid transfection 54

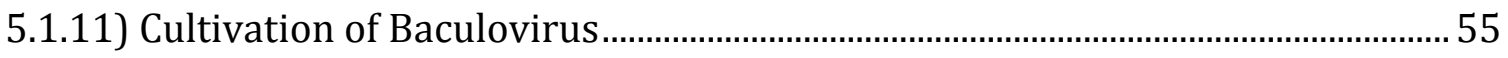

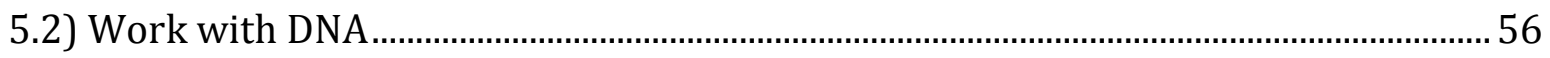

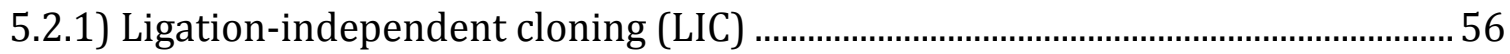

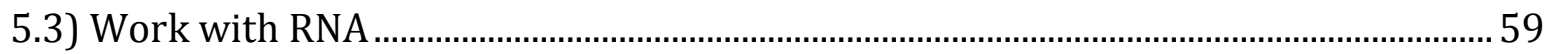

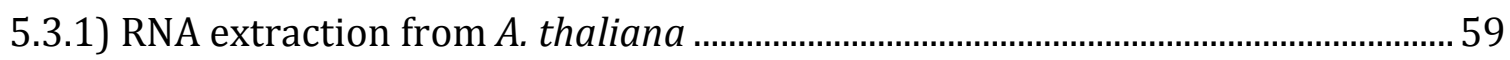

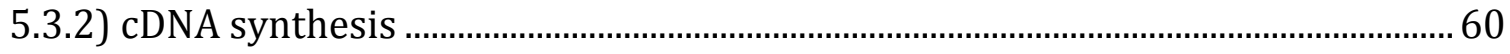

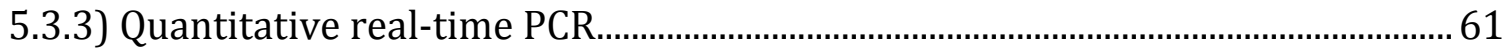

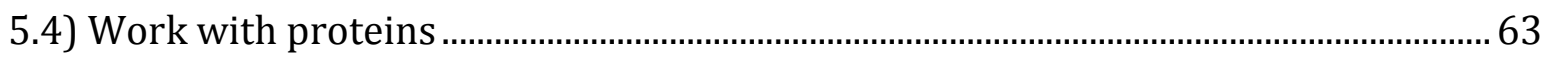

5.4.1) Preparation of protein extracts from A. thaliana in urea buffer.......................... 63

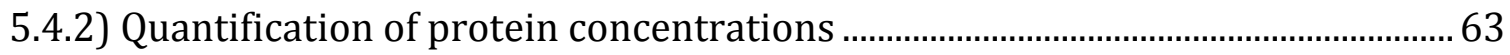

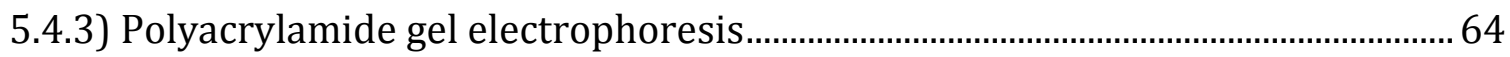

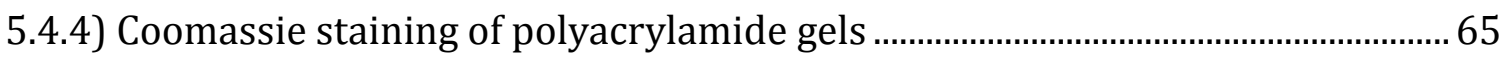

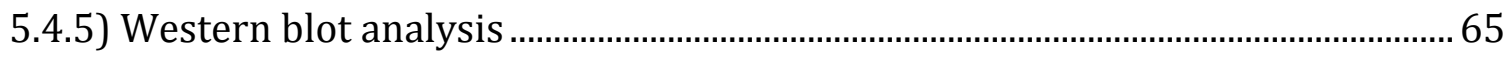

5.4.6) ÄKTA-based purification of strep-MBP-NPR1 from insect cells........................... 66

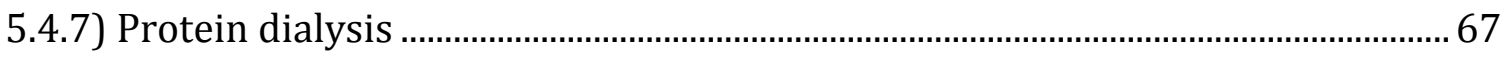

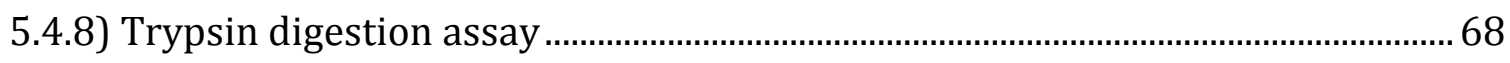

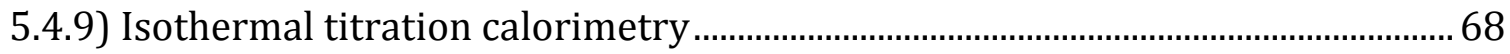

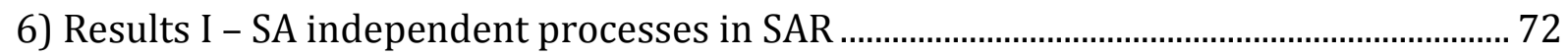

6.1) The transcriptional regulation of FMO1 in Psm-infected SAR leaves is SAindependent, but NPR1-dependent. 
6.2) The conserved arginine residue (R432) in NPR1 is required for the induction of FM01

6.3) SA-independent FMO1 induction requires TGA2/5/6 and TGA1/4 78

6.4) SA-independent FMO1 induction requires ALD1 80

6.5) NHP treatment leads to SA synthesis which is required for full induction of FMO1 and $P R 1$ 82

6.6) NPR1 and TGA1/4 are required for the induction of FMO1 in NHP-treated plants 85

6.7) NHP treatment leads to NPR1 accumulation 88

6.8) EDS1 is required for the regulation of FMO1 by NHP 89

6.9) Primary infection with Psm potentiates the effect of NHP treatment 92 6.10) Using Isothermal Titration Calorimetry (ITC) to study the NPR1 - SA interaction

7) Results II - ROXY19 is involved in the repression of ORA59. 95

7.1) The induction of ROXY19 expression during local and systemic immunity is partially SA-dependent. 95

7.2) ROXY19 is dispensable for the plant immune response against Psm. 97

7.3) ROXY19 is a negative regulator of ORA59 expression. 99

7.4) The single cysteine (C186) in TGA2 is not required for the repression of ORA59

7.5) SA-mediated repression of PDF1.2 during SAR does not depend on ROXY18/19

7.6) ROXY19 is not involved in the repression of xenobiotic stress response genes..105

7.7) roxy18 roxy19 mutants are more resistant to $B$. cinerea infections .106

8) Discussion I - SA-independent processes in SAR 109

8.1) A mobile signal confers SA-independence on FMO1 expression in SAR leaves....109 
8.2) SA-independent FMO1 expression requires the SA receptor NPR1 and the NPR1interacting TGA transcription factors

8.3) Is NPR1 a receptor for NHP?

8.4) EDS1 is required for activation of target genes by NHP

8.5) ITC might be used to characterize the interaction between NPR1 and SA.

9) Discussion II - ROXY18 and ROXY19 are involved in SA-mediated repression of ORA59.

9.1) ROXY18 and ROXY19 are not involved in the regulation of FMO1

9.2) ROXY19 and ROXY18 are involved in repression of ORA59 promoter activity....120

10) Summary I - SA-independent processes in systemic acquired resistance (SAR) .....124

11) Summary II - The glutaredoxin ROXY19 is a negative regulator of the JA/ETdependent defense pathway

11) References.

12) Abbreviations

13) Supplementary figures 140

14) Acknowledgements 142 



\section{1) Introduction I - SA-independent processes in SAR}

\section{1) Defense responses in plants}

\subsection{1) Local immune responses}

Being sessile, plants have developed a sophisticated immune response to prevent pathogen infection. The first layer of defense is in the form of physical barriers such as wax layers on the surface and a robust cell wall, apart from which antimicrobial enzymes and metabolites present in the apoplast prevent the proliferation of pathogens (Heath, 2000; Thordal-Christensen, 2003). Adapted pathogens can bypass the physical and chemical barriers robust as they may be (Jones and Dangl, 2006). To restrict growth during the early stage of pathogen colonization, plants have evolved methods to recognize pathogens and activate defense response. Pathogens such as bacteria and fungi have highly conserved and indispensable "molecular patterns" that are essential for growth and mobility. Throughout co-evolution, plants have adapted to recognize these Pathogen/Microbe Associated Molecular Patterns (PAMPs/MAMPs).

Plants recognize PAMPs via pattern recognition receptors (PRRs) located in the plasma membrane; recognition of PAMPs leads to the initiation of PAMPTriggered Immunity (PTI) (Boller and Felix, 2009). PRRs in plants can be broadly classified into two groups, the Receptor-Like Kinases (RLKs) and the ReceptorLike Proteins (RLPs). Both types of receptors contain an extracellular domain and a transmembrane domain. The main difference between the RLKs and RLPs is the presence of an intracellular kinase domain in the RLKs, which is absent in RLPs. Based on the domains or motifs in the extracellular domain, PRRs can be classified into different subfamilies: leucine-rich repeat (LRR) domain, lysine motifs (LysM), lectin domain, or epidermal growth factor (EGF)-like domain (Couto and Zipfel, 2016). One of the best-studied PRRs is the LRR-RLK Flagellin Sensing 2 (FLS2) receptor (Chinchilla et al., 2006). FLS2 mediated recognition of the conserved peptide flg22 present in the N-terminal part of bacterial flagellin leads to the formation of a heterodimer between FLS2 and BRASSINOSTEROID 
INSENSITIVE 1-ASSOCIATED RECEPTOR KINASE 1 (BAK1) (Chinchilla et al., 2007). The complex so formed phosphorylates BOTRYTIS-INDUCED KINASE 1 (BIK1), which then contributes to the generation of Reactive Oxygen Species (ROS) and initiation of the Mitogen Associated Protein Kinase (MAPK) pathway (Felix et al., 1999; Lu et al., 2010). Upon activation, the MAPK pathway phosphorylates transcription factors, which in turn regulate the expression of immune related genes. The genes so regulated include the critical factors involved in biosynthesis of phytohormones and secondary metabolites (Kim and Zhang, 2004; Mao et al., 2011). To circumvent the defense response that is initiated, pathogens have developed strategies that rely on effector molecules (virulence factors), which target components of the PTI response, leading to Effector Triggered Susceptibility (ETS) (Dou and Zhou, 2012; Guo et al., 2019; Wang and Wang, 2018).

Bacterial pathogens such as Pseudomonas syringae rely on the Type III secretion system to deliver effectors into the host cells (Buttner, 2016; Collmer et al., 2000). Arabidopsis is highly susceptibile to Pseudomonas syringae pv. tomato DC3000 (Pto DC3000) as the bacteria carry a repertoire of effectors to dampen the PTI response. The bacterial effector AvrPto for instance, is known to suppress immunity in tomato and Arabidopsis. As AvrPto suppresses the induction of a wide range of cell wall-associated genes, it was initially thought to function by blocking cell wall-associated defense responses. However, many of the early PTI marker genes are also suppressed by AvrPto, suggesting that the effector targets early events in the PTI signaling cascade (Abramovitch et al., 2003).

To retaliate against the ETS caused by pathogen invasion, plants have evolved disease resistance genes (R genes), which encode Nucleotide binding site (NB)Leucine-Rich Repeat (LLR) proteins (NLRs) (Jones and Dangl, 2006; Van de Weyer et al., 2019). The NLRs are divided into two types, TIR-NB-LRR (TNL), which contains an N-terminal Toll and Interleukin like -1 (TNL) domain, and the CC-NB-NLR (CNL), which contains a Coiled-Coil-domain (Bonardi and Dangl, 2012; Maekawa et al., 2011). 
The main difference between TNLs and CNLs is the requirement for activation of the signaling cascade. TNLs require the nucleo-cytoplasmic proteins ENHANCED DISEASE SUSCEPTIBILITY (EDS1) and PHYTOALEXIN DEFICIENT 4 (PAD4) as well as SENESCENCE ASSOCIATED GENE 101 (SAG101) for the initiation of signaling responses (Aarts et al., 1998), whereas CNLs rely on NON-RACESPECIFIC-DISEASE RESISTANCE 1 (NDR1) (Coppinger et al., 2004).

Recognition of effectors by NLR proteins leads to the initiation of defense responses that involve oxidative burst, accumulation of the phytohormone salicylic acid, induction of defense genes, and a form of localized cell death called Hypersensitive Response (HR) (Dempsey and Klessig, 1994; Durner and Klessig, 1996; Han and Hwang, 2017).

There is an overlap between the defense responses initiated upon the activation of PTI and ETI. The PTI triggered by the PAMP flg22 and the ETI response initiated upon the recognition of the effector AvrRpt2 are lost in the quadruple mutant of delayed dehiscence2 (dde2), ethylene insensitive2 (ein2), phytoalexin4 (pad4), and salicylic acid induction deficient2 (sid2), which is simultaneously deficient in JA, ethylene, PAD4, and SA signalling. This shows that the signalling networks are extensively shared between ETI and PTI. However, during ETI, they are expressed more strongly and with a faster kinetics (Mine et al., 2018).

\subsection{1) Systemic Acquired Resistance}

Apart from activation of local defense responses, recognition of pathogens by plant cells leads to a heightened state of alertness against further attacks in the uninfected parts of the plant. This form of immunity, called Systemic Acquired Resistance (SAR), provides long-lasting protection against a broad spectrum of pathogens. Ross et al. first reported the phenomenon of SAR, showing that the HR triggered by Tobacco Mosaic Virus (TMV) results in the plant being more resistant towards subsequent infections (Ross et al., 1961). The initiation of the HR response was considered a prerequisite for the establishment of SAR. 
However, the publication from Mishina et al. showed that SAR could be triggered by treatment of local leaves with PAMPs such as flg22 and lipopolysaccharides without inducing the HR response (Mishina and Zeier, 2007). The SAR response consists of three main phases -1 ) generation of the mobile signals, 2) transport of the mobile signals, and 3) recognition of the mobile signals followed by downstream signal activation. The identity of the mobile signals involved in SAR has remained elusive for a long time; some of the initial contenders included salicylic acid (SA), methyl salicylate (MeSA), azelaic acid (AzA), and dihydroabietinal (DA) (Chaturvedi et al., 2012; Gaffney et al., 1993; Jung et al., 2009; Liu et al., 2011; Vernooij et al., 1994b).

In a grafting experiment performed with tobacco by Vernooji et al., it was shown that when wildtype scions were grafted on transgenic rootstocks expressing the SA-degrading bacterial enzyme NahG, the SAR response was still viable. However, in a reciprocal graft with wildtype rootstock and transgenic scion, SAR was not realized suggesting that $\mathrm{SA}$ is not the mobile signal, but that SA accumulation is a prerequisite in the distal leaves for the establishment of SAR (Vernooij et al., 1994a).

\section{2) SA as a critical player in the establishment of SAR}

Transcriptome analysis using uninfected systemic leaves have shown that the induction of a large sector of the SAR-related genes depend on salicylic acid (Bernsdorff et al., 2016). Moreover, the SA biosynthesis and signaling mutants are impaired in the establishment of systemic immunity. Though SA is not a mobile signal, it is of utmost importance for the processes leading to SAR. In this section, we will concentrate on the biosynthesis of SA and the downstream signaling that follows. 


\subsection{1) Salicylic acid biosynthesis}

SA biosynthesis requires the end product of the shikimate pathway - chorismate. Chorismate (CA) is converted into SA via two independent pathways: the isochorismate (IC) and the phenylalanine ammonia-lyase (PAL) pathway (Ribnicky et al., 1998; Vlot et al., 2009).

In the PAL pathway, L phenylalanine formed from chorismate is converted to trans-cinnamic acid (t-CA) by the PAL enzyme, after which ABNORMAL INFLORESCENCE MERISTEM 1 (AIM1) converts t-CA to benzoic acid (BA) (Richmond and Bleecker, 1999). The enzymatic reactions that are involved in the conversion of BA to SA are still unknown.

In the isochorismate pathway, chorismate is converted into isochorismate by ISOCHORISMATE SYNTHASE 1 (ICS1). Isochorismate is then conjugated to the amino acid glutamate by the enzyme AvrPhpB SUSCEPTIBLE 3 (PBS3). Following conjugation by PBS3, isochorismate-9-glutamate nonenzymatically decomposes into SA. The contribution of PBS3 in SA biosynthesis was recently reported by Rekhter et al. and Torrence-Spence et al. (Rekhter et al., 2019; Torrens-Spence et al., 2019). It was further shown that ENHANCED PSEUDOMONAS SUSCEPTIBILITY 1 (EPS1) could bolster SA production by the stimulating the decay of isochorismate-9-glutamate.

Following pathogen infection, the pal quadruple mutant shows 50\% accumulation of SA as compared to wildtype, whereas the ics1 ics2 double mutant only shows $10 \%$ of SA accumulation (Wildermuth et al., 2001). This shows that while the PAL pathway contributes to SA accumulation, the ICS pathway is the major contributor to SA accumulation after pathogen infection.

Both ICS1 and ICS2 are localized in the chloroplast (Garcion et al., 2008; Wildermuth et al., 2001), implying that conversion of chorismate to isochorismate takes place in the plastids. Studies have revealed that mutation in the ABC transport protein ENHANCED DISEASE SUSCEPTIBILITY 5 (EDS5) leads to a drastically low accumulation of SA (Nawrath et al., 2002). Moreover, EDS5 is 
localized in the chloroplast envelope, suggesting that it is involved in the transport of SA or its precursor from the chloroplast to the cytosol. In the report by Rekhter et al., it was shown that the conversion of isochorismate to SA takes place in the cytosol. Taken together, it would imply that isochorismate, which is synthesized in the plastids, is transported to the cytosol by EDS5, where it is converted to SA by PBS3 and EPS1.

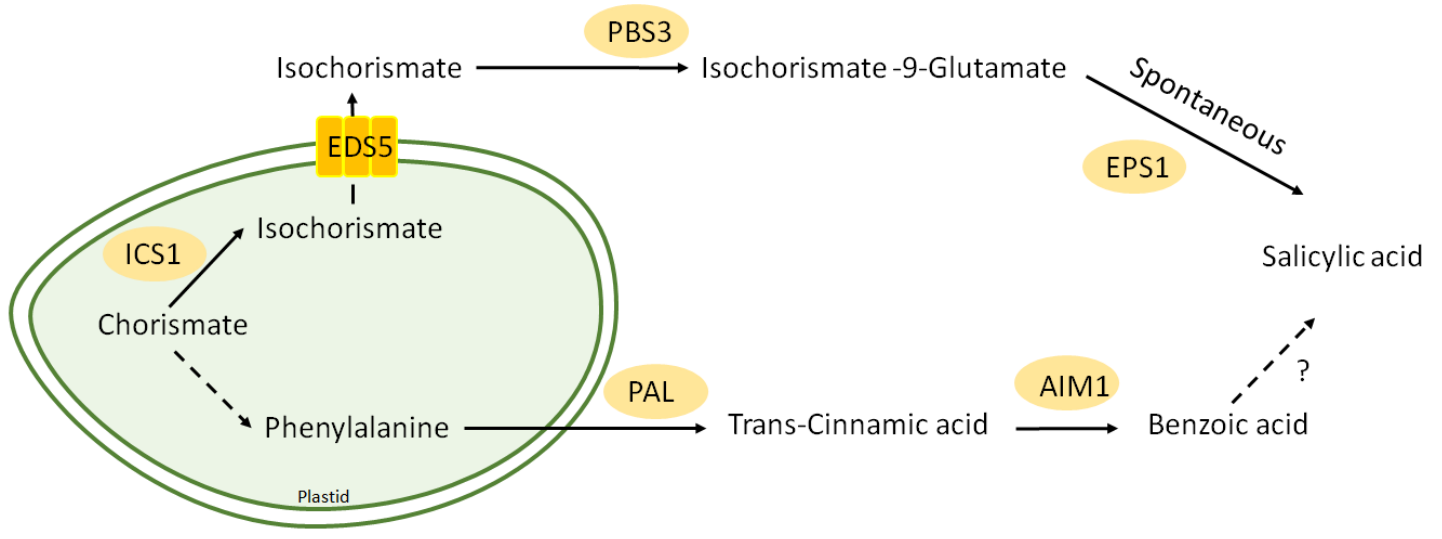

Figure 1: Salicylic acid biosynthesis pathway. In the phenylalanine ammonialyase (PAL) pathway, PAL converts phenylalanine to trans cinnamic acid, which is converted to benzoic acid by ABNORMAL INFLORESCENCE MERISTEM (AIM1). The step resulting in the production of salicylic acid from benzoic acid is unknown. In the isochorismate synthase (ICS) pathway, ICS1 converts chorismate to isochorismate in the plastid. The MATE transporter EDS5 transports isochorismate from the plastid to the cytosol, where AvrPhpB SUSCEPTIBLE 3 (PBS3) conjugates it to glutamate. The IC-9Glu thus formed spontaneously degrades to SA; EPS1 aids this process. Figure modified from Huang et al,2019(Huang et al., 2019).

\subsection{2) Transcriptional regulation of SA biosynthesis}

Due to the detrimental effect that SA accumulation has on plant fitness, the biosynthesis of SA is tightly regulated. Pathogen infection leads to an increase in the levels of cytosolic calcium $\mathrm{Ca}^{2+}$ (Boudsocq and Sheen, 2013; Poovaiah et al., 2013; Wang et al., 2009). The rise in Calcium is decoded by calcium sensor proteins such as calmodulin (CaM). Binding of CaM further regulates the target proteins leading to gene induction (Kim et al., 2009). The CaM binding protein CALMODULIN BINDING PROTEIN 60G (CBP60G) and its close homolog SAR 
DEFICIENT 1 (SARD1) have been shown to bind to the ICS1 promoter and regulate its expression (Sun et al., 2015).

The TEOSINTE BRANCHED1/CINCINNATA/PROLIFERATING CELL FACTOR (TCP) transcription factors TCP8 and TCP9 are involved in the regulation of SA biosynthesis. TCP8 and 9 bind to the ICS1 promoter and the tcp 8 tcp 9 double mutant is impaired in the accumulation of SA after Pseudomonas syringae pv. maculicola (Psm) infection. Moreover, the interaction between TCP8/TCP9 and SARD1 has been shown using the bimolecular fluorescence complementation (BiFC) assay. A complex consisting of SARD1 and TCP8/TCP9 likely regulates the transcription of ICS1 (Wang et al., 2015).

Furthermore, WRKY transcription factors play an essential role in the transcriptional regulation of ICS1. It was shown that overexpression of WRKY28 and WRKY46 in Arabidopsis protoplasts leads to an upregulation of ICS1 expression (van Verk et al., 2011). Using chromatin immunoprecipitation (ChIP) assays, the binding of WRKY28 to the ICS1 promoter was proven at least in protoplasts. The WRKY transcription factors WRKY8 and WRKY48 have a specific role in the effector-triggered ICS1 induction, as revealed by the observation that the wrky8 and wrky48 mutants were impaired in the expression of ICS1 upon infection with Pst DC3000 avrRpm1 and avrRpt2 but not Pst DC3000 (Gao et al., 2013).

The CaM binding transcription factor CALMODULIN BINDING TRANSCRIPTION ACTIVATOR 3/ SIGNAL RESPONSIVE GENE 1 (CAMTA3/SR1) binds to the ICS1 promoter to repress its induction (Kim et al., 2013). Moreover, the CAMTA transcription factor also represses EDS1, which regulates ICS1 via an unknown mechanism. Upon pathogen infection, the CAMTA transcription factor is linked to ubiquitin-mediated degradation by SIGNAL RESPONSIVE GENE 1 INTERACTING PROTEIN (SR1IP), thereby releasing ICS1 from the repression (Zhang et al., 2014). 
The mechanistic aspect of how the opposing effects of CAMTA and SARD1 translate to the expression of ICS1 is currently unknown. Gene expression analysis performed using a sard1 camta123 combination mutant would help in addressing if blocking the repressive effect of CAMTA on ICS1 alone is sufficient for the induction of gene expression.

\subsection{3) Perception of SA by NON EXPRESSOR OF PATHOGENESIS-RELATED GENES 1 (NPR1)}

A forward genetic screen using transgenic plants expressing the bacterial reporter gene ß-glucuronidase (GUS) driven by the BETA-1,3-GLUCANASE 2 (BGL2) gene promoter was used to identify mutants that did not show GUS expression upon treatment with the SA analog 2,6-dichloro-isonicotinic acid (INA) (Cao et al., 1994). This screen identified NPR1 as a crucial regulator of the signaling cascade activated upon SA.

A decade after the discovery of NPR1, Wu et al. reported the role of NPR1 as a SA receptor (Wu et al., 2012). Using equilibrium dialysis (EqD), the authors showed that NPR1 binds SA with a Kd of $140 \mathrm{nM}$ but does not bind structurally related analogs of SA. Moreover, they showed that NPR1 binds SA via two cysteine residues ( $\mathrm{Cys}^{521 / 529}$ ) and that the transition metal $\mathrm{Cu}^{2+}$ is crucial for binding of NPR1 to SA. Using size exclusion chromatography to separate the NPR1-SA complex from unbound SA, Ding et al. also reported SA binding to NPR1. However, the importance of Cys ${ }^{521 / 529}$, which is not conserved in NPR1 from other plant species, was questioned. Instead, arginine 432 was defined as being crucial for in vitro SA binding and in vivo function of NPR1 (Ding et al., 2018).

NPR1 is present primarily as oligomers in the uninduced cells, held together by disulfide bonds. Accumulation of SA causes a redox shift in the cells, resulting in the monomerization of NPR1 (Mou et al., 2003). The thioredoxin TRXh5 aids the monomerization process. Mutation in residues Cys82 or Cys216 resulted in the constitutive monomerization and nuclear localization of NPR1, which in turn led 
to somewhat induced transcript levels of PATHOGENESIS RELATED GENE 1 (PR1) (Tada et al., 2008b). However, upon SA treatment, the PR1 transcript levels further increased in the transgenic line carrying NPR1 with the mutated cysteines. Moreover, the pathogen resistance phenotype of the transgenic lines was not as strong as the phenotype observed in SA-treated wild type plants (Tada et al., 2008b). This suggests that nuclear localization of NPR1 is sufficient, but further SA-related processes are required for the actuation of PR1 induction.

Though two independent labs have reported on the SA binding feature of NPR1, with data showing the requirement for two unrelated residues, the exact mechanism that facilitates the binding is still largely unknown.

Following translocation into the nucleus, NPR1 interacts with the TGACG BINDING FACTORs (TGAs) transcription factors leading to the induction of SARrelated genes (Kinkema et al., 2000; Zhang et al., 1999; Zhou et al., 2000). Given that NPR1 does not have a DNA binding domain, its interaction with TGA transcription factors is a crucial link between SA perception and gene induction.

\subsection{4) Class I and II TGA transcription factors as mediators of NPR1 function during SAR}

The Arabidopsis genome encodes for ten TGA transcription factors, which are divided into five classes. TGA factors are basic leucine zipper (bZIP) proteins that bind to variants of the TGACGTCA palindrome, with the first five base pairs (TGACG) being sufficient for binding. For this thesis, we will concentrate on class I and class II TGA transcription factors.

In a linker scanning mutagenesis study designed to find regulatory elements within the PR1 promoter, it was found that LS7 (Linker Scanning 7) which corresponds to a mutation of the TGACG sequence $640 \mathrm{bp}$ upstream of the transcription start site, led to a failure of $P R 1$ induction following SA treatment (Lebel et al., 1998). A year following the publication of the LS7 element, Zhang et al. showed that the class II TGA transcription factors - TGA2, TGA5, and TGA6 - 
can interact with NPR1 and can bind to the PR1 promoter. Moreover, a tga 2 tga5 tga6 triple knockout mutant behaved similarly to the npr1 mutant in terms of loss of $P R 1$ induction and SAR deficiency (Zhang et al., 2003). This suggested that class II TGA transcription factors and NPR1 work in the same pathway leading to the induction of PR1 and SAR.

Conflicting data are available for the SAR phenotype of the tga1 tga4 mutant: one study claims that the tga1 single but not the tga1 tga4 double mutant is compromised with respect to SAR establishment after secondary infection with Psm (Shearer et al., 2012), while another study reports compromised SAR of the tga1 tga4 mutant after secondary infection with Hyaloperonospora arabidopsidis (Sun et al., 2018). Using the tag1 tga4 npr1 mutant, it was demonstrated that the TGA1 and TGA4 acts in an NPR1-independent manner, as the triple mutant showed higher susceptibility as compared to the tga1 tga4 double mutant and the npr1 mutant. More recently, Sun et al. showed that TGA1 is a regulator of SARD1 expression. As mentioned above, SARD1 regulates the transcription of the SA biosynthesis gene ICS1. Levels of SA were low in the tga1 tga4 double mutant and could be complemented on the introduction of a TGA1 genomic construct into the tga1 tga4 double mutant (Sun et al., 2018).

The interaction between TGA1 and NPR1 was not as strong as the interaction between NPR1 and TGA2 in yeast two-hybrid experiments (Zhou et al., 2000). Depres et al 2003 reported that reduction of a disulfide bond within TGA1 was required for the interaction with NPR1 (Despres et al., 2003). Moreover, Lindermayr et al showed that nitrosylation of TGA1 facilitated the interaction as well (Lindermayr et al., 2010). Subsequently, the DNA binding affinity of TGA1 was enhanced, but the functional relevance of this had remained obscure especially in view of the data of Shearer et al who published that TGA1 mainly acts independently of NPR1. 


\section{3) Pipecolic acid and N-hydroxy pipecolic acid (NHP) - new}

\section{players in the field}

Pipecolic acid is a product of lysine catabolism and is present in a wide range of plants, animals, and microorganisms. The evidence for the pathway from lysine to pipecolic acid was first provided by Rothstein and Miller (1954). Injecting radiolabelled lysine into rats led to highly radioactive pipecolic acid, establishing that pipecolic acid is a metabolic product of lysine in rats (Rothstein and Miller, 1954). Around the same time, a similar experiment done on Phasaeolus vulgaris showed that, much like in the animal system, pipecolic acid is derived from lysine in plants as well (Lowy, 1953).

\subsection{1) Pipecolic acid and NHP biosynthesis}

Návarová and colleagues showed the accumulation of pipecolic acid upon Psm infection. They further explained that the aminotransferase AGD2-LIKE DEFENSE RESPONSE PROTEIN (ALD1) plays an essential role in the conversion of L-lysine to pipecolic acid (Navarova et al., 2012). The expression of ALD1 is strongly induced upon pathogen infection; moreover, the ald1 mutant did not accumulate pipecolic acid.

In two independent studies by Ding et al. and Hartmann et al., the enzymatic function of ALD1 was elucidated. It was shown that the first step in the biosynthesis of pipecolic acid from l-lysine involves ALD1-mediated transfer of the $\alpha-\mathrm{NH}_{2}$ group of l-lysine to acceptor oxoacids resulting in the cyclic ketimine 1,2-dehydropipecolic acid (1,2-DP) [alias $\Delta^{1}$-piperideine-2-carboxylic acid $\left(\Delta^{1}\right.$ P2C)], which is likely present in equilibrium with 2,3-DP (Ding et al., 2016; Hartmann et al., 2017). The two studies further reported that 1,2-DP is reduced to pipecolic acid by the enzyme SAR DEFICIENT 4 (SARD4). The protein $\mu$ crystallin (CRYM) in mammals catalyzes the reduction of 1,2-DP using NADH or NADPH as reduction equivalents. The sequences of CRYM and SARD4 are similar 
in that they possess a Rossman NAD-binding domain. It was shown that the sard4 mutant is impaired in the local and systemic accumulation of pipecolic acid.

However, at later time points, the sard4 mutants showed a moderate accumulation of pipecolic acid. This suggests the presence of additional pathways leading from DP to pipecolic acid.

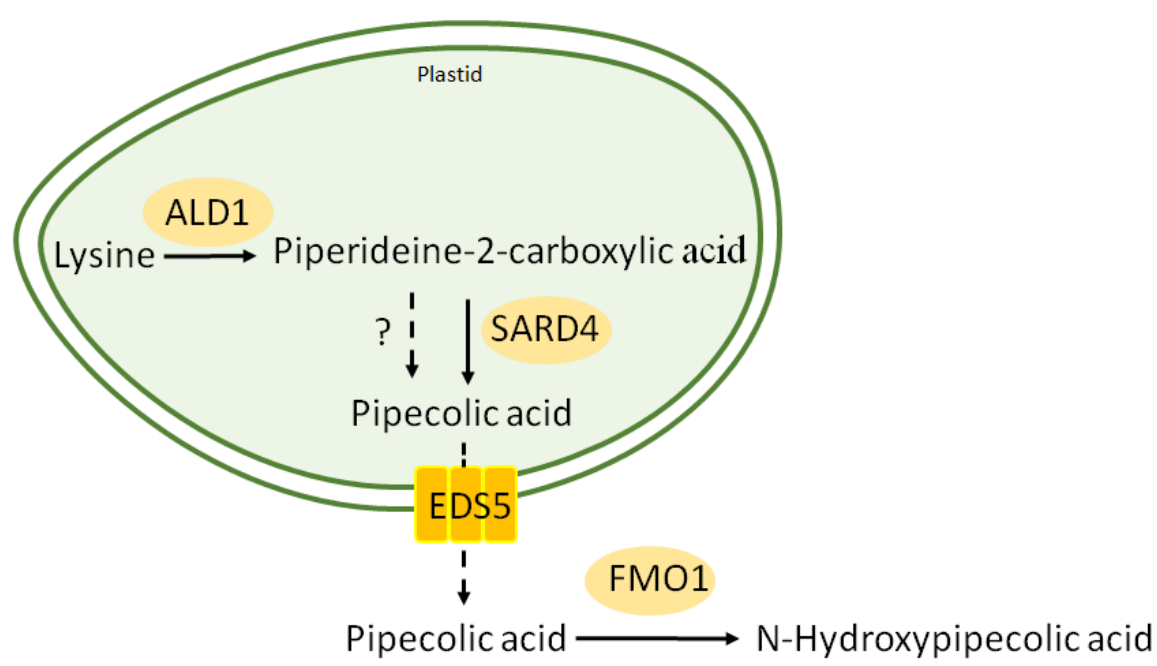

Figure 2 : NHP biosynthesis pathway. Lysine is converted to pipecolic acid in a twostep process catalyzed by ALD1 and SARD4 (along with other yet unknown reductase). The pipecolic acid formed is likely transported out of the plastid by EDS5. Once in the cytosol, FMO1 catalyzes the conversion of pipecolic acid to N-hydroxy pipcolic acid. modified from Huang et al.,2019 (Huang et al., 2019).

More recently, in a landmark finding by Hartmann et al., the role of FLAVIN DEPENDENT-MONOOYGENASE 1 (FMO1) in the conversion of pipecolic acid to NHP was reported. Using GC-MS analysis, the authors were able to discover a metabolite that accumulated upon pathogen infection in the wild type plants, but not in the ald1 or fmo1 mutants (Hartmann et al., 2018). Moreover, feeding experiments with deuterated pipecolic acid led to the detection of deuterated NHP, confirming that NHP is synthesized from pipecolic acid. Around the same time, an independent report by Chen et al. displayed that on transient expression 
of FMO1 in tobacco, followed by pipecolic acid feeding, the formation of NHP was observed (Chen et al., 2018).

In a very recent study by Rekhter et al., it was shown that the accumulation of NHP following UV treatment is substantially impaired in the eds5 mutant. The low accumulation of NHP in the eds5 mutant could not be rescued upon the application of SA, suggesting that the observation is not due to the inability of the eds5 mutant to accumulate SA. However, upon application of pipecolic acid, the mutants showed a moderate accumulation of NHP (Rekhter et al., 2019a). Thus, EDS5 seems to be involved in the transport of pipecolic acid from the plastid to the cytosol, where it is converted to NHP by FMO1.

Overall, the biosynthesis of NHP from l-lysine consists of three steps:

1) $\alpha$-transamination of l-Lysine to DP by ALD1

2) reduction of DP to pipecolic acid by SARD 4 and other yet unknown factors

3) N-hydroxylation of pipecolic acid to NHP by FMO1

\subsection{2) Transcriptional regulation of NHP biosynthesis and downstream signaling}

The key genes involved in the biosynthesis of NHP- ALD1, SARD4, and FMO1 are all induced in the systemic leaves during SAR. Treating plants with pipecolic acid primes for the induction of the genes mentioned above. Priming by pipecolic acid is absent in the fmo1 mutant, suggesting that NHP is the inducing agent. Moreover, treatment with NHP has shown to trigger the expression of the three NHP pathway genes.

The nucleocytoplasmic proteins EDS1 and PAD4 are involved in the transcriptional regulation of NHP biosynthesis genes. Conversely, elevated levels of pipecolic acid trigger the induction of EDS1 and PAD4, suggesting that EDS1 and PAD4 are involved in a defense amplification loop that is initiated upon the 
biosynthesis of NHP. This assumption is further strengthened by the observation that the susceptibility shown in the pad4 mutant cannot be rescued by supplying the plants with pipecolic acid.

The transcription factor SARD1, which regulates the SA biosynthesis gene ICS1, also binds to the promoters of $A L D 1$ and FMO1 (Sun et al., 2015). The sard1 cbp60g double mutant is impaired in the induction of ALD1 and FMO1 in response to Psm infection. Furthermore, Sun et al. showed that TGA1 and TGA4 are regulators of SARD1 expression. The tga1 tga4 double mutant was impaired in the induction of $A L D 1$ and showed impairment in the accumulation of pipecolic acid upon pathogen infection.

NPR1 does not regulate the induction of FMO1 in the local leaves. Contrarily, NHP was shown to over-accumulate in the local leaves of npr1 after Psm infection, suggesting that NPR1 has a negative effect on the NHP accumulation in the local leaves (Hartmann et al., 2018). However, during systemic infection, NPR1 seems to play an important role in the regulation of FMO1 as the npr1 mutant is impaired in the systemic induction of FMO1 (Bernsdorff et al., 2016).

\section{4) ENHANCED DISEASE SUSCEPTIBILITY 1 (EDS1) as an essential component of the transcriptional reprogramming during SAR}

The nucleo-cytoplasmic protein EDS1 along with its interaction partners PHYTOALEXIN DEFICIENT 4 (PAD4) and SENESCENCE-ASSOCIATED GENE 101 (SAG101) form a critical hub required for basal and TNL-triggered immune responses (Falk et al., 1999; Feys et al., 2005; Wiermer et al., 2005).

EDS1 and PAD4 are conserved across a wide range of seed plants and contain a highly conserved N-terminal lipase-like domain and a C-terminal EP domain (EDS1-PAD4 domain). The $N$-terminal domain has homology to an $\alpha / \beta$ hydrolase fold, consisting of eight $\beta$-sheets connected by $\alpha$-helices. The $\alpha / \beta$ hydrolase fold containing protein DEFENDER AGAINST DEATH (DAD2) has been shown to bind 
strigolactone (SL) and activate the SL-mediated signaling cascade (Hamiaux et al., 2012). The N-terminal domain also possesses the Ser-His-Asp catalytic triad, which is characteristic of the lipase domain, but no catalytic activity has been detected in EDS1 (Rietz et al., 2011; Wagner et al., 2013). Moreover, the EDS1PAD4 catalytic residues are dispensable for immune regulatory functions, as shown by mutational analysis of the triads (Wagner et al., 2013).

The current model places EDS1 and PAD4 upstream of SA biosynthesis. A feedback loop involving SA further induces EDS1 and PAD4, resulting in an enhanced expression of defense-related genes. Apart from bolstering the SAdependent signaling cascade, EDS1 is also engaged in SA-independent signaling. In a screen performed to identify SA-independent functions of EDS1, it was reported that EDS1 is involved in the transcriptional regulation of FMO1, irrespective of local SA accumulation (Bartsch et al., 2006).

Moreover, in a series of petiole exudates transfer experiment performed by Breitenbach et al 2014 , the induction of $P R 1$ was absent on infiltration of the wildtype plants with petiole exudates collected from Psm-infected eds1 mutant plants. Similarly, the induction was also missing when petiole exudates collected from wild type plants were infiltrated into eds1 mutant (Breitenbach et al., 2014). Hence, EDS1 is involved in the generation as well as the perception of the mobile signal during systemic resistance.

\section{5) Interconnection between SA and NHP synthesis}

SA treatment leads to the induction of genes involved in SA biosynthesis; this takes place in an NPR1-dependent manner. Besides, the induction of SARD1 is also upregulated upon SA treatment, which then contributes to the induction of ICS1, EDS5, and PBS3 (Sun et al., 2015). SA accumulation also leads to the induction of genes involved in SA catabolism processes such as DOWNY MILDEW RESISTANT 5 (DMR5) and DMR6-LIKE OXYGENASE 1 (DLO1) (Zhang et al., 2017). 
Moreover, SA accumulates to higher levels in the locally infected npr1 mutant leaves, suggesting that NPR1 exerts a negative effect on SA accumulation (Delaney et al., 1995).

Similarly, pipecolic acid and NHP treatment results in the induction of NHP biosynthesis genes. Hartmann et al. showed that Pip treatment results primes the induction of $A L D 1, E D S 1, P A D 4$, and FMO1, which are all involved in the biosynthesis of NHP. As mentioned above, the Pip-mediated induction of all the genes mentioned above were absent in the fmo1 mutant, suggesting that NHP is the effective metabolite. Interestingly, SA and NHP seem to bolster the biosynthesis of each other. SA treatment can induce the expression of ALD1, FMO1, and SARD4, whereas Pip treatment results in the induction of ICS1, EDS5, and PBS3 (Chen et al., 2018; Hartmann et al., 2018).

The current model holds that during SAR, SA accumulates in the systemic leaves in a Pip/NHP-dependent manner, the accumulation of SA then results in the amplification of the expression of SAR-related genes. While the induction of a large portion of SAR-related genes is dependent on SA, around one-quarter of the genes show a SA-independent induction. The two most notable genes that are induced independently of SA are the genes involved in NHP biosynthesis ALD1 and FMO1. This suggests that the NHP biosynthesis pathway functions independently of SA and that the NHP signaling pathway is responsible for the induction of SA-independent genes during SAR. However, the accumulation of SA is a prerequisite for the actuation of a full SAR response and to turn on genes such as PR1, which is strictly dependent on SA. 


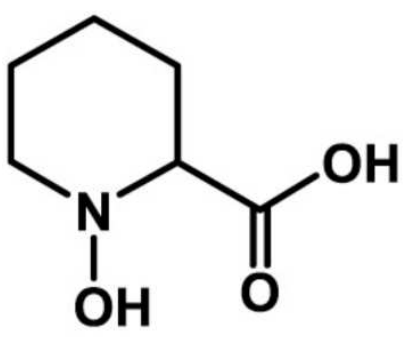

$\mathrm{N}$-Hydroxypipecolic acid

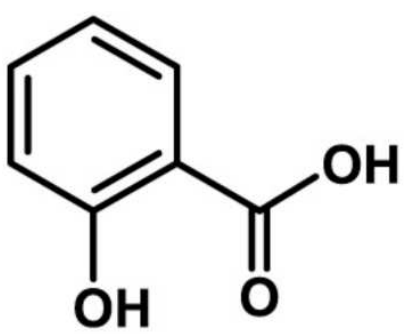

Salicylic acid

Figure 3: Molecular structures of N-hydroxypipecolic acid (NHP) and salicylic acid (SA). The molecular structures of NHP and SA resemble each other, especially with respect to the presence of similarly arranged carboxylic acid and hydroxyl functional groups (taken from Hartmann et al., 2018).

NHP biosynthesis is an absolute requirement for the establishment of SAR, as exemplified by the lack of SAR in the ald 1 and fmo1 mutants. In the absence of Pip biosynthesis, the induction of all SAR-related genes, including ICS1, is hampered. However, supplying the ald1 and fmo1 mutants with SA results in the induction of $P R 1$ and elevated resistance to Psm. 


\section{2) Introduction II - The glutaredoxin ROXY19 is a negative regulator of the JA/ET-dependent defense pathway}

\section{1) Glutaredoxins (GRXs) in Arabidopsis}

Glutaredoxins (GRXs) are small ubiquitous proteins which are characterized by the presence of a structural motif, the thioredoxin (TRX) fold (Rouhier et al., 2008a; Rouhier et al., 2008b). This fold consists of $4 \beta$-sheets and $3 \alpha$-helices (Martin, 1995; Qi and Grishin, 2005). GRXs catalyze the reversible reduction of cysteine disulfide bridges and protein-glutathione (GSH) mixed disulfide bonds using glutathione (GSH) as a cofactor. The catalytic site consisting of a $\mathrm{CxxC} / \mathrm{S}$ motif resides at the $\mathrm{N}$ terminus of $\alpha$ helix 1 (Rouhier et al., 2008b). GRXs employ either the monothiol or the diothiol mechanism to reduce protein disulfide bridges or glutathione-mixed disulfides (Deponte, 2013; Lillig et al., 2008). Both mechanisms start with the reduction of the substrates and oxidation of GRX. Subsequently, the GRX is reduced at the expense of GSH (Lillig et al., 2008).

During the monothiol mechanism, the N-terminal active cysteine of the GRX attacks the protein disulfide or the glutathione-mixed disulfide. In a situation where glutathione-mixed disulfide is the substrate, the $\mathrm{N}$-terminal active cysteine of the GRX takes over the glutathione moiety from the substrate in a disulfide exchange reaction. Consequently, the $\mathrm{N}$-terminal active cysteine gets covalently linked to the cysteine residue of glutathione; the substrate protein now forms a free thiol. If a protein disulfide bridge serves as a substrate, the disulfide is resolved by formation of an intermolecular disulfide bridge between the GRX and the substrate. The intermolecular disulfide bridge is then reduced by a GSH, leading to a thiol on the substrate protein and a glutathionylated GRX. The GRX is regenerated with the help of another molecule of glutathione, resulting in the formation of glutathione disulfide (GSSG) and a reduced GRX. 
A

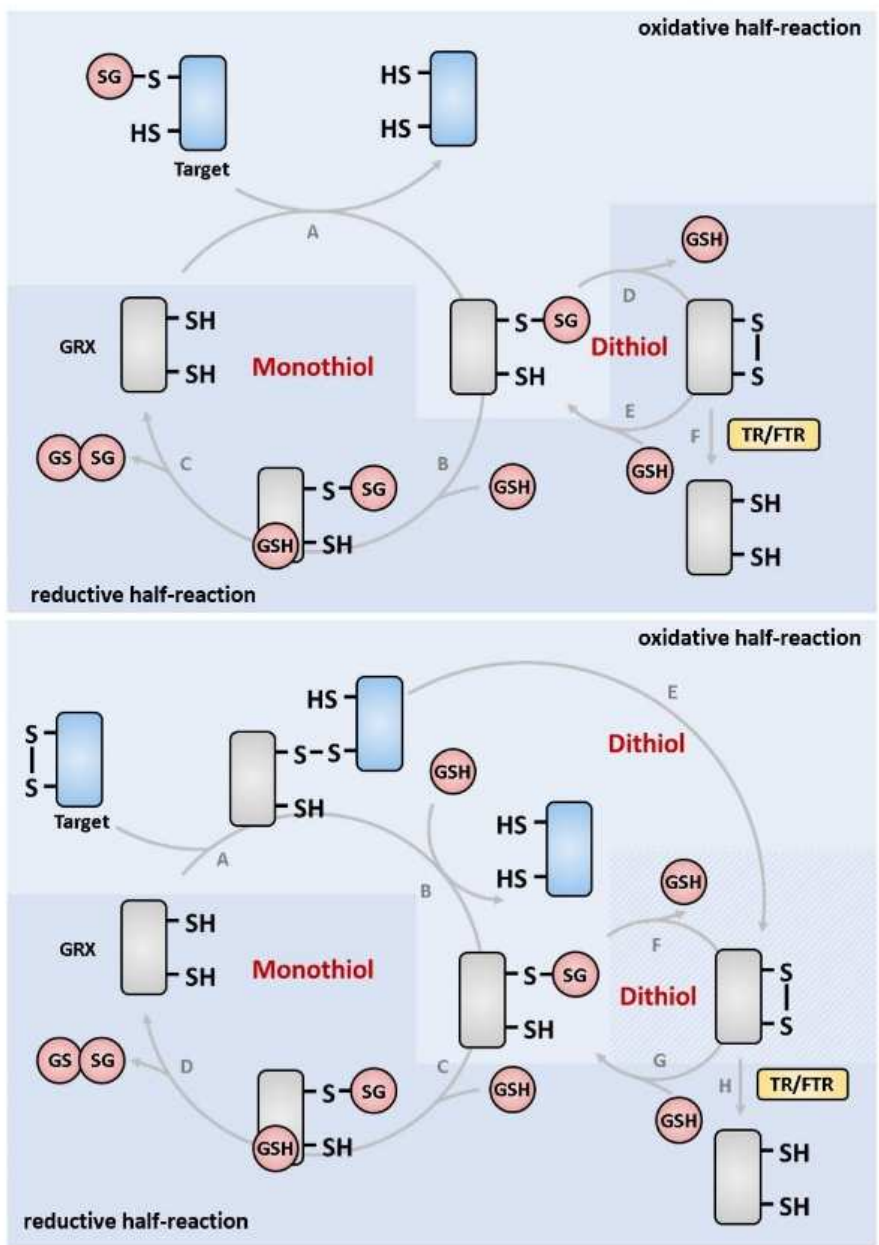

Figure 4: Reaction mechanisms used by GRXs. GRXs deglutathionylate proteins using the monothiol or the dithiol mechanism. (A) During deglutathionylation via the monothiol mechansim, a reduced GRX takes over the glutathione moiety (-SG) from the glutathionylated target protein (step $A$ ) and then deglutathionylates itself with the help of another glutathione molecule (GSH), releasing glutathione disulfide (GSSG, steps B and $C$ ). When using the dithiol mechanism for degluathionylation, the glutaredoxin is deglutathionylated forming an intramolecular disulfide (step $D$ ), which is subsequently resolved by two molecules of GSH (steps $E, B$, and $C$ ) or thioredoxins (TR) or ferredoxin thioredoxin reductase (FTR, step $F$ ). (B) shows the reduction of disulfide bridges in target proteins via the mono- or dithiol mechanism. During the dithiol mechanism, an intramolecular disulfide is formed within the GRX in the course of a disulfide exchange (steps $A$ and $E$ ), whereas in the monothiol mechanism, the GRX undergoes glutathionylation and deglutathionylation only (steps $A$ to $D$ ). However, after step $B$ of the monothiol mechanism, the GRX can also enter the dithiol mechanism (steps $F$, $G$ and $H$ ). During the dithiol mechanism, the disulfide bridge is either resolved by TR or FTR (step $H$ ) or via two molecules of GSH, releasing GSSG (steps $G, C$ and $D$ ). When viewed in terms of the reaction sequence steps $A, B, F, H$ or $A, B, F, G, C, D$, the reaction product of step $F$ belongs to the reductive half-reaction. When viewed from the reaction sequence beginning with steps $A$ and $E$, it belongs to the oxidative half reaction. The schemes and legend were taken from Rouhier et al. (2008), Deponte (2013), and Ukuwela et al. (2018) and modified. Taken from Katrin Treffon PhD thesis 2019 
The GSSG is further reduced into two molecules of GSH by GSH reductase, using the reducing power of NADPH. When using the dithiol mechanism for degluathionylation, the GRX is deglutathionylated forming an intramolecular disulfide, which is subsequently resolved by two molecules of GSH, thioredoxins, or ferredoxin thioredoxin reductase.

GRXs are also involved in the formation of Fe-S clusters in the mitochondrial matrix and in the transfer of Fe-S clusters to proteins. A fundamental function of Fe-S clusters is to transfer electrons, apart from which Fe-S clusters also serve as a source of iron and sulphur, and as sensors of cellular changes in $\mathrm{Fe}$ concentrations to facilitate gene expression. The number of GRXs in plants is exceptionally high as compared to other organisms. The Arabidopsis genome encodes for 31 glutaredoxins, which based on the active sites, are separated into three classes.

1) The CPYC-type (class I) consisting of six members: GRXC1, GRXC2, GRXC3, GRXC4, GRXC5 and GRXS12

2) The CGFS-type (class II) consisting of four members: GRXS14, GRXS15, GRXS16 and GRXS17

3) The plant-specific CC-type (class III or ROXY) consisting of 21 members (Li et al., 2009; Rouhier et al., 2004).

\subsection{1) Class I GRXs in plants}

The CPYC-type is well studied in Arabidopsis. It encodes four cytosolic CPYC GRXs: GRXC1, GRXC2, GRXC3 and GRXC4, and at least one chloroplastic CPYC GRX named GRXS12 (Couturier et al., 2013). Genetic studies revealed that GRXC1 and GRXC2 are indispensable for plant viability (Riondet et al., 2012; Rouhier et al., 2007). Moreover, GRXC2 was shown to glutathionylate BRASSINOSTEROID INSENSITIVE 1 (BRI1)-ASSOCIATED RECEPTOR-LIKE KINASE 1 (BAK1) 
suggesting the role of GRXC2 in the BAK1-related signaling cascade (Bender et al., 2015).

\subsection{2) Class II GRXs in plants}

CGFS-GRXs are found in all prokaryotes and eukaryotes (Xing et al., 2006). Four members of this class, GRXS14, GRXS15, GRXS16 and GRXS17 are present in Arabidopsis (Moseler et al., 2015). The catalytic activity of the CGFS-type GRXs was found to be weak in the classical biochemical assay for oxidoreductase activity using the artificial substrate bis(2-hydroxyethyl)disulfide (HEDS) (Stroher et al., 2016). All plant CGFS-type GRXs tested so far have the ability to incorporate [2Fe-2S] clusters (Bandyopadhyay et al., 2008; Knuesting et al., 2015; Moseler et al., 2015), which fits to the established role of this type of GRXs as necessary components for the maturation of FeS proteins in other organisms. In vivo evidence for this function has been obtained for the Arabidopsis mitochondrial GRXS15 (Moseler et al., 2015).

\subsection{3) The plant-specific class III GRXs}

While class I and class II GRXs are present in eukaryotes and prokaryotes, class III GRXs are found only in higher land plants (Couturier et al., 2009; Rouhier et al., 2008b). The number of class I and II GRX genes remained constant through evolution in plants and does not differ drastically from the numbers in other organisms. The class III GRX genes on the other hand underwent multiple duplication events resulting in a higher number in the land plants. Biochemical characterization of class III GRX is limited due to the difficulty in purification. Still, poplar GRXS7.2 was successfully purified from E.coli. It displayed typical features of Fe-S cluster binding (such as brownish color and specific UV/visible light absorption) (Couturier et al., 2010) and poor oxidoreductase activity in the HEDS assay. 
Arabidopsis encodes for 21 CC-type GRXs, also termed ROXYs. The active site motif of ROXYs is characterized by a conserved CC motif (consensus: CC (M/C) (C/S/G)) (Xing et al., 2006; Ziemann et al., 2009). All tested CC-type GRXs interact with TGA transcription factors and a functional link between ROXYs and TGAs has been established in development- and immunity-related processes (Zander et al., 2012).

\section{2) Regulation of developmental processes by ROXY1 and the TGA transcription factor PERIANTHIA (PAN)}

Genetic analysis of the roxy1 mutant revealed the role of ROXY1 in flower development (Xing et al., 2005). While wildtype plants form four petals, the roxy1 mutant forms in average only 2.5 petals. Interestingly, the interacting TGA transcription factor PAN is involved in the determination of flower organ number. The pan single mutant and pan roxy1 double mutant show formation of an extra petal, suggesting that ROXY1 acts as a negative regulator of PAN. Complementation analysis showed that the first cysteine in the active site of ROXY1 is required for petal development, the glycine residue in the GSH binding site is also critical for its function. The cysteine residue Cys340 was shown to be required for PAN function. Therefore it was postulated that ROXY1 may target and inhibit PAN function by means of redox modification (Xing et al., 2005).

Moreover, ROXY1 and its homolog ROXY2 are involved in anther development, Histological analysis of the roxy1roxy2 anthers showed the formation of smaller anthers without the presence of pollen grains (Xing and Zachgo, 2008). The tga9 tga10 double mutant shows a phenotype similar to roxy1 roxy2 mutant with respect to anther development. Since the expression pattern of TGA9 and TGA10 overlaps with the expression pattern of ROXY1 and ROXY2 and since TGA9 and TGA10 directly interact with ROXY proteins in yeast and in plant cell nuclei, it was speculated that ROXY1 and ROXY2 might influence the regulatory functions of TGA9 and TGA10. 


\section{3) Regulation of immune-related genes by ROXY18 and ROXY19}

Microarray analysis revealed that ROXY18 is induced on exposure to abiotic and biotic stresses. Treatment with SA was shown to induce ROXY18, whereas JA treatment led to repression of ROXY18. The inverse pattern of induction seen upon SA and JA treatment led La Camera et al to speculate that ROXY18 is a susceptibility gene induced upon infection with the necrotrophic pathway Botrytis cinerea (La Camera et al., 2011). They further showed that ROXY18 isinduced in an SA-dependent manner upon B. cinerea infection and that the roxy18 mutant is more resistant in infection assays with $B$. cinerea. The expression of JA/ET-controlled immune related genes that are induced upon $B$. cinerea infection showed a similar pattern between wildtype and the roxy18 mutant. The mechanism as to how ROXY18 participates in the immune signaling activated on infection by $B$. cinerea is currently unknown.

ROXY19, which is a close homolog of ROXY18, also shows an SA-dependent induction pattern (Zander et al., 2012). However, JA treatment also leads to the induction of ROXY19 (Zander et al., 2014). Ectopic expression of ROXY19 led to higher susceptibility to $B$. cinerea. This observation could be correlated with the repression of OCTADECANOID-RESPONSIVE ARABIDOPSIS AP2/ERF domain protein 59 (ORA59), seen when ROXY19 is ectopically expressed (Zander et al., 2014). ORA59 is a key transcription factor required for the JA/ET-activated defense response effective against necrotrophic pathogens (Pre et al., 2008). A loss of function evidence for the role of ROXY19 has not yet been described. As SA antagonises the JA/ET pathway and since ROXY19 is induced by SA, it was speculated to play a role in the cross talk between SA and JA/ET.

Zander et al. reported that ethylene introduces the requirement of class II TGA transcription factors for the induction of ORA59, while the induction mediated by JA takes place independently of TGA transcription factors. The antagonistic effect exerted by SA on the JA pathway requires the TGA transcription factors. Firstly, 
TGAs are required for the SA mediated induction of ROXY19; moreover, the ectopically expressed ROXY19 cannot repress ORA59 in the absence of TGA transcription factors (Zander et al., 2014).

The negative effect of SA on the JA/ET pathway likely takes place by the repression of ORA59. The promoter region of ORA59 contains a TGA binding site, and in vivo binding of TGA2 to the promoter of ORA59 has been shown by Zander et al. Based on the evidence from plants ectopically expressing ROXY19, it was postulated that SA-induced ROXY19 is recruited to the ORA59 promoter by TGA transcription factors, which leads to inhibition of promoter activity (Zander et al., 2014).

As the conserved cysteine residue was shown to be essential for the functionality of the TGA transcription factor PAN, it was initially speculated that during the SAJA/ET crosstalk, the cysteine residue in TGA2 is targeted by ROXY19 for redox modifications, leading to repression of its function. Recently, Uhrig et al showed that ROXY19 interacts with the transcriptional repressor TOPLESS (TPL) through the conserved C-terminal ALWL motif. Furthermore, the ability of ROXY19 to form a ternary complex with TPL and TGA2 at the TGA binding site was shown. Based on these observations, it was speculated that ROXY19 interacts with TGA2 and functions as an adaptor that recruits TPL to the promoter site, thereby leading to repression of the target gene (Uhrig et al., 2017). However, the role of the conserved active centre in this process has remained enigmatic. 


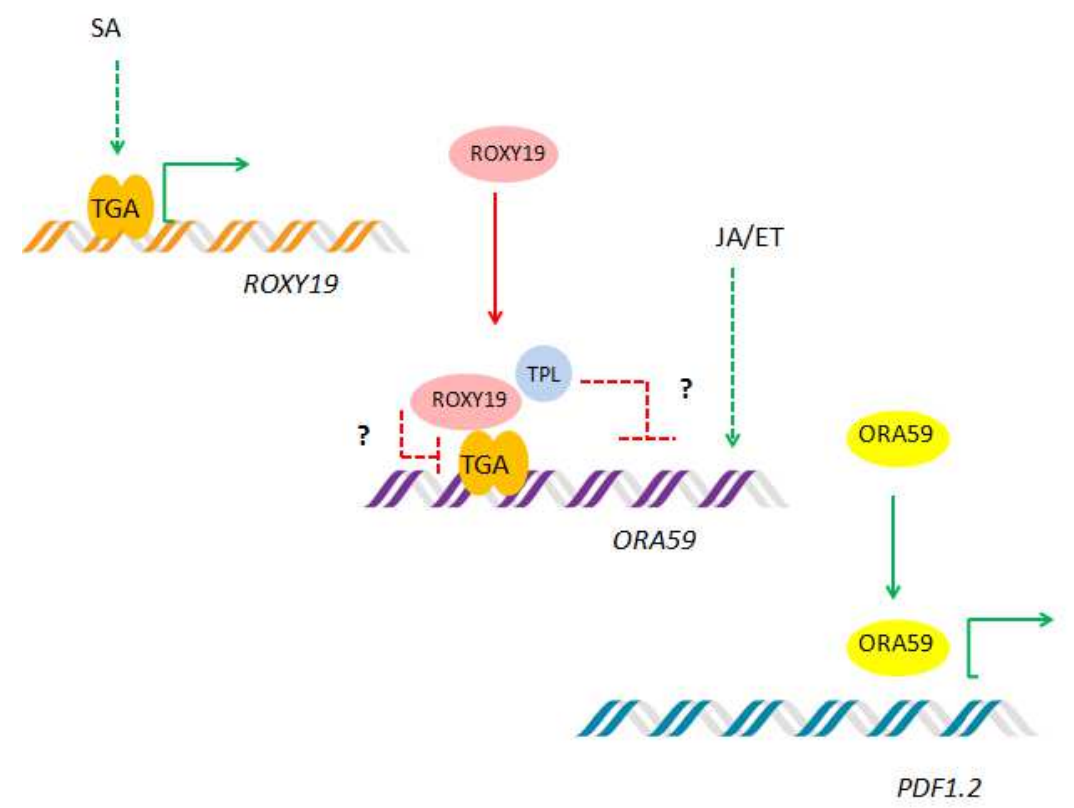

Figure 5: Putative role of ROXY19 in the SA-JA cross-talk. SA induces ROXY19 transcription in a TGA-dependent manner. Upon induction, ROXY19 is recruited to the promoter of the gene encoding for the JA/ET signaling master regulator ORA59 by TGA transcription factor. ROXY19 inhibits the promoter activity of ORA59 through a yet unknown mechanism. Interaction observed between TGA2-ROXY19 and the transcriptional repressor TPL suggests that the ternary complex so formed might be involved in the repression or ORA59. ORA59 activates the marker gene of the JA/ET defense cascade PDF1.2 as well as other genes of the pathway. 


\section{3) Aim of this thesis}

Transcriptome analysis performed on uninfected systemic leaves showed that a quarter of SAR related genes are regulated independently of the phytohormone salicylic acid (SA) (Bernsdorff et al., 2016). The aim of this study was to unravel the SA-independent processes during SAR. Specifically, the question of how FMO1 is regulated in the systemic leaves during SAR was addressed. Apart from this, a second objective of the thesis was to characterize the function of SA-inducible glutaredoxins (GRXs) ROXY18 and ROXY19 during pathogen responses. 


\section{4) Materials}

\section{1) Organisms}

Table 1: Bacterial strains

\begin{tabular}{l|l}
\hline Species & Reference \\
\hline Pseudomonas syringae pv. maculicola ES4326 & Whalen et al. 1991 \\
\hline E.coli DH10 $\mathrm{EMBacY}$ & $\begin{array}{l}\text { Department Cramer, MPI } \\
\text { Biophysical Chemistry, } \\
\text { Göttingen }\end{array}$ \\
\hline
\end{tabular}

Table 2: Insect cells

\begin{tabular}{l|l|l}
\hline Insect cells & Line & Reference \\
\hline Trichoplusia $n i$ & High FiveTM & Thermo Fisher Scientific \\
\hline Spodoptera frugiperda & IPLB-Sf21AE & Vaughn JL et al. 1977 \\
\hline
\end{tabular}

Table 3: Arabidopsis thaliana genotypes

\begin{tabular}{l|l}
\hline Genotype & Reference \\
\hline Col-0 & NASC stock no. N1902 \\
\hline tga1 tga4 & Kesarwani et al., 2007 \\
\hline tga2 tga5 tga6 & Zhang et al., 2003 \\
\hline npr1-1 & Cao et al., 1994 \\
\hline sid2-2 & Wildermuth et al., 2001 \\
\hline sid2 npr1 & AG Gatz \\
\hline sid2 tga2 tga5 tga6 & K. Rindermann PhD thesis 2010 \\
\hline sid2 tga1 tga4 & M. Muthreich PhD thesis 2014 \\
\hline sid2 ald1 & Bernsdorff et al. 2016 \\
\hline roxy18 roxy19 & Parker et al. 1996 \\
\hline tga256 35S:TGA2C186S & AG Gatz \\
\hline
\end{tabular}




\section{2) Primers}

Table 4: Primers used for qRT-PCR analysis - custom made primers

\begin{tabular}{|c|c|c|}
\hline Primer & forward sequence $\left(5^{\prime}-3^{\prime}\right)$ & reverse sequence $\left(5^{\prime}-3^{\prime}\right)$ \\
\hline$U B Q 5$ & GACGCTTCATCTCGTCC & GTAAACGTAGGTGAGTCCA \\
\hline$P R 1$ & $\begin{array}{l}\text { CTGACTTTCTCCAAACAACT } \\
\text { TG }\end{array}$ & $\begin{array}{l}\text { CAAACTAAACAATAAACCATACCAT } \\
\text { AA }\end{array}$ \\
\hline SARD1 & TCAAGGCGTTGTGGTTTGTG & CGTCAACGACGGTATGTTTC \\
\hline PDF1.2 & CTTGTTCTCTTTGCTGCTTTC & CATGTTTGGCTCCTTCAAG \\
\hline TRXh5 & GAATTGCAAGCTGTTGCTCA & CACCGACAACACGATCAATG \\
\hline $\operatorname{SnRK2.8}$ & CCTGAAGTGCTCTCCACGAA & GCATTCATCCGAAACTCGAA \\
\hline
\end{tabular}

Table 5: Primers used for qRT-PCR analysis - QuantiTect ${ }^{\circledR}$

\begin{tabular}{l|l}
\hline Primer & Quantitect ID \\
\hline FMO1 & QT00861427 \\
\hline ICS1 & QT00893473 \\
\hline ORA59 & QT00852054 \\
\hline
\end{tabular}

Table 6: Primers used for ligation independent cloning (LIC)

\begin{tabular}{l|l|l}
\hline Primers & Sequence & \\
\hline NPR1 Frw & TACTTCCAATCCAATGCAATGGACACCACCATTGATGG \\
\hline NPR1 Rev & $\begin{array}{l}\text { TTATCCACTTCCAATGTTATTATCACCGACGACGATGAG } \\
\text { AG }\end{array}$ \\
\hline
\end{tabular}

\section{3) Plasmids}

Table 7: Plasmids

\begin{tabular}{l|l}
\hline Plasmid Name & Description \\
\hline 438C & $\begin{array}{l}\text { Expression vector for insect cells harboring the coding } \\
\text { sequence of a strep-MBP tag for N-terminal fusion }\end{array}$ \\
\hline
\end{tabular}




\section{4) Antibodies}

Table 8: Antibodies

\begin{tabular}{l|l|l}
\hline Antibody & Description & Source \\
\hline Anti-NPR1 & $\begin{array}{l}\text { Polyclonal antibody against NPR1 from } \\
\text { rabbit }\end{array}$ & Agrisera \\
\hline Anti-rabbit & HRP conjugated anti rabbit IgG from goat & LifeTechnologies \\
\hline
\end{tabular}

\section{5) Chemicals}

Table 9: Chemicals

\begin{tabular}{|c|c|}
\hline Chemical & Company \\
\hline $32 \%$ Hydrochloric acid (HCL) & Roth \\
\hline Acetic acid $\left(\mathrm{CH}_{3} \mathrm{COOH}\right)$ & Roth \\
\hline Acetone $\left(\mathrm{C}_{3} \mathrm{H}_{6} \mathrm{O}\right)$ & Roth \\
\hline Agar for bacteria & Roth \\
\hline Agar for plants & Roth \\
\hline Agarose & Sigma-Aldrich \\
\hline Ammonium thiocyanate $\left(\mathrm{NH}_{4} \mathrm{SCN}\right)$ & Sigma-Aldrich \\
\hline Ammoniumperoxodisulfate $\left(\mathrm{NH}_{4}\right)_{2} \mathrm{~S}_{2} \mathrm{O} 8$ & Roth \\
\hline Ampicillin & Roth \\
\hline Bromophenol blue & Roth \\
\hline Calcium chloride dihydrate $\left(\mathrm{CaCl}_{2} .2 \mathrm{H}_{2} \mathrm{O}\right)$ & Roth \\
\hline Chloroform $\left(\mathrm{CHCL}_{3}\right)$ & Roth \\
\hline Coomassie Brilliant Blue G 250 & Merck \\
\hline D-Desthiobiotin & IBA \\
\hline d-Desthiobiotin & IBA \\
\hline Deoxyadenosine triphosphate (dATP) & LifeTechnologies \\
\hline Deoxycytidine triphosphate (dCTP) & LifeTechnologies \\
\hline Deoxyguanosine triphosphate (dGTP) & LifeTechnologies \\
\hline Deoxythymidine triphosphate (dTTP) & LifeTechnologies \\
\hline Desiccator grease & Roth \\
\hline Dimethylsulfoxide (DMSO) & Roth \\
\hline Dipotassium hydrogen phosphate $\left(\mathrm{K}_{2} \mathrm{HPO}_{4}\right)$ & Roth \\
\hline $\begin{array}{l}\text { Disodium ethylenediaminetetraacetate dihydrate } \\
\text { (EDTA) }\end{array}$ & Sigma \\
\hline Disodium hydrogen phosphate dihydrate $\left(\mathrm{KH}_{2} \mathrm{PO}_{4}\right)$ & Roth \\
\hline Disodium phosphate $\left(\mathrm{Na}_{2} \mathrm{HPO}_{4}\right)$ & Roth \\
\hline DNA size standard & Thermo Scientific \\
\hline Ethanol $\left(\mathrm{C}_{2} \mathrm{H}_{5} \mathrm{OH}\right)$ & Nordbrand \\
\hline
\end{tabular}


Materials

\begin{tabular}{|c|c|}
\hline Glycerol $\left(\mathrm{C}_{3} \mathrm{H}_{8} \mathrm{O}_{3}\right)$ & Roth \\
\hline Guanidine thiocyanate $\left(\mathrm{C}_{2} \mathrm{H}_{6} \mathrm{~N}_{4} \mathrm{~S}\right)$ & Roth \\
\hline HRP substrates for Western Blot detection & Thermo Scientific \\
\hline Isopropanol $\left(\mathrm{C}_{3} \mathrm{H}_{8} \mathrm{O}\right)$ & Roth \\
\hline Isopropyl- $\beta$-D-thiogalactopyranoside (IPTG) & Roth \\
\hline Liquid $\mathrm{N}_{2}$ & Westfalen AG \\
\hline Magnesium chloride $\left(\mathrm{MgCl}_{2}\right)$ & Roth \\
\hline Magnesium sulfate heptahydrate $\left(\mathrm{MgSO}_{4} .7 \mathrm{H}_{2} \mathrm{O}\right)$ & Roth \\
\hline Methanol $\left(\mathrm{CH}_{3} \mathrm{OH}\right)$ & Merck \\
\hline Monopotassium phosphate $\left(\mathrm{KH}_{2} \mathrm{PO}_{4}\right)$ & Roth \\
\hline Peptone & BioScience \\
\hline Phenol $\left(\mathrm{C}_{6} \mathrm{H}_{5} \mathrm{OH}\right)$ & Sigma \\
\hline Potassium hydroxide (KOH) & Roth \\
\hline Protein size standard for SDS-PAGE & Thermo Scientific \\
\hline Rifampicin & Duchefa Biochemie \\
\hline Sodium dodecyl sulfate (SDS) & Roth \\
\hline Skimmed milk powder & SUCOFIN \\
\hline Sodium acetate $\left(\mathrm{C}_{2} \mathrm{H}_{3} \mathrm{NaO}_{2}\right)$ & Roth \\
\hline Sodium chloride $(\mathrm{NaCl})$ & Roth \\
\hline Sodium dihydrogen phosphate $\left(\mathrm{NaH}_{2} \mathrm{PO}_{4}\right)$ & Roth \\
\hline Sodium hydroxide $(\mathrm{NaOH})$ & Roth \\
\hline Spectinomycin & Duchefa Biochemie \\
\hline Sucrose $\left(\mathrm{C}_{12} \mathrm{H}_{22} \mathrm{O}_{11}\right)$ & Duchefa Biochemie \\
\hline Tetramethylethylenediamine (TEMED) & Roth \\
\hline TRIS & Roth \\
\hline Tris(2-carboxyethyl) phosphine (TCEP) & Sigma-Aldrich \\
\hline Trisodium citrate dihydrate $\left(\mathrm{Na}_{3} \mathrm{C}_{6} \mathrm{H}_{5} \mathrm{O}_{7}\right)$ & Roth \\
\hline Trypsin & SERVA \\
\hline Tween-20 & Roth \\
\hline Urea $\left(\mathrm{CH}_{4} \mathrm{~N}_{2} \mathrm{O}\right)$ & Roth \\
\hline Yeast extract & Roth \\
\hline 30 \% Acrylamide-Bisacrylamide solution (ratio 37.5:1) & Roth \\
\hline 1,4-Dithiotreit (DTT) & Roth \\
\hline
\end{tabular}




\section{6) Kits and enzymes}

Table 10: Kits and enzymes

\begin{tabular}{|c|c|}
\hline Kit/Enzyme & Source \\
\hline $\begin{array}{l}\text { Amersham }^{\mathrm{TM}} \text { ECL }^{\mathrm{TM}} \text { Anti-rabbit IgG, } \\
\text { Horseradish Peroxidase-Linked Antibody }\end{array}$ & GE Healthcare \\
\hline $\begin{array}{l}\text { BIOTAQ DNA Polymerase } 5 \mathrm{u} / \mu \mathrm{l} \text { with } 50 \mathrm{mM} \\
\mathrm{MgCl}_{2} \text { stock solution and } 10 \mathrm{x} \text { NH4 reaction } \\
\text { buffer }\end{array}$ & Bioline \\
\hline $\begin{array}{l}\text { DNase I, RNase-free, } 1 \mathrm{U} / \mu \mathrm{l} \text { with } \mathrm{MgCl}_{2} \text { for } \\
\text { DNase I, 10x Reaction buffer and } 50 \mathrm{mM} \text { EDTA }\end{array}$ & Thermo Scientific \\
\hline Fluorscein dye $1 \mathrm{mM}$ in DMSO & BioRad Laboratories \\
\hline NucleoSpin $®$ Gel and PCR clean-up & Macherey \& Nagel \\
\hline $\begin{array}{l}\text { Phusion High-Fidelity DNA Polymerase }(2 \mathrm{U} / \mu \mathrm{l}) \\
\text { with } 5 x \text { Phusion Buffer (contains } 7.5 \mathrm{mM} \mathrm{MgCl}_{2} \text { ) }\end{array}$ & Thermo Scientific \\
\hline Pierce 660nm Protein Assay Reagent & Thermo Scientific \\
\hline $\begin{array}{l}\text { Revert Aid H Minus Reverse Transcriptase } \\
200 \mathrm{U} / \mu \mathrm{l} \text { with } 5 \mathrm{x} \text { Reaction Buffer for real time }\end{array}$ & Thermo Scientific \\
\hline $\operatorname{SspI}(10 \mathrm{U} / \mu \mathrm{l})$ & Thermo Scientific \\
\hline SuperSignal ${ }^{\mathrm{TM}}$ West Femto kit & Thermo Scientific \\
\hline $\begin{array}{l}\text { SYBR@ Green I Nucleic Acid Stain 10000x in } \\
\text { DMSO }\end{array}$ & $\begin{array}{l}\text { Cambrex BioScience } \\
\text { Rockland }\end{array}$ \\
\hline T4 DNA Polymerase $5 \mathrm{u} / \mu \mathrm{l}$ & Fermentas \\
\hline
\end{tabular}




\section{5) Methods}

\section{1) Work with organisms}

\subsection{1) Arabidopsis thaliana surface sterilization and growth conditions}

A. thaliana seeds were surface sterilized with chlorine gas in a desiccator using vapor phase sterilization method. Open tubes containing seeds were placed in a desiccator along with a beaker containing $50 \mathrm{ml}$ of $12 \%$ sodium hypochlorite solution and $2.5 \mathrm{ml}$ of $32 \%$ hydrochloric acid. Vacuum pressure of approximately $200 \mathrm{mBar}$ was applied and the seeds were left for four hours in the desiccator. Before the storage or use of surface-sterilized seeds, the tubes were left open for approximately 30 mins under the air hood to let the chlorine gas evaporate. The sterilized seeds were transferred to round pots (diameter $6 \mathrm{~cm}$ ) containing soil (Fruhstorpfer Topferde Typ T Struktur 1 Fein) which was soaked twice with 0.2 \% Wuxal Super (Manna, Ammerbuch-Pfäffingen, Germany). The seeds were stratified in the cold room for two days, and the trays were transferred to the $12 \mathrm{~h}$ light climate chambers with light intensity fixed at $100 \mu \mathrm{mol} \mathrm{m} \mathrm{m}^{-2} \mathrm{~s}^{-1}$ and $65 \%$ relative humidity.

\subsection{2) Systemic Acquired Resistance (SAR) experiments}

SAR was performed on 4.5-week-old-plants. The petioles of three lower leaves were marked with a medium-size permanent marker (Faber-Castell). $10 \mathrm{mM}$ $\mathrm{MgCl}_{2}$ or Pseudomonas syringae pv. maculicola ES4326 (Psm) (O.D. 0.005) was infiltrated into the abaxial side of the leaves using a needleless syringe. Two days after the infiltration of the lower leaves, three upper leaves were infiltrated with Psm (O.D. 0.005) for gene expression assays or Psm (O.D. 0.0001) for bacterial growth assays. 


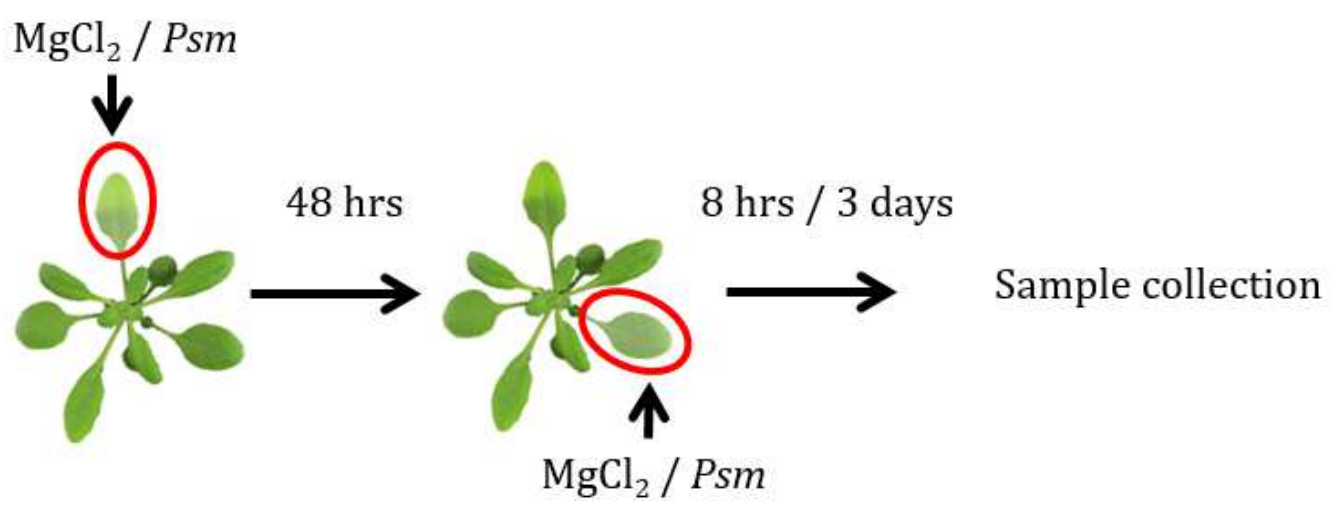

Figure 6: Schematic representation of Systemic Acquired Resistance (SAR) experiment. Three lower leaves were infiltrated with $10 \mathrm{mM} \mathrm{MgCl}$ or Psm (O.D. 0.005 ) diluted in $10 \mathrm{mM} \mathrm{MgCl}_{2}$. Two days after the primary infection, upper leaves were infiltrated with $10 \mathrm{mM} \mathrm{MgCl}_{2}$ or Psm (O.D. 0.005); the upper leaves were collected after 8 hours for gene expression studies. For bacterial growth assays, the upper leaves were infiltrated with Psm (O.D. 0.0001), and samples were collected after 3 days.

For gene expression analysis, leaves were collected in a screw cap tube containing a $5 \mathrm{~mm}$ metal bead and immediately transferred to liquid nitrogen. For bacterial growth assay,_leaf discs were collected in tubes containing $200 \mu \mathrm{l}$ of $10 \mathrm{mM} \mathrm{MgCl} 2$ and a single $5 \mathrm{~mm}$ metal bead.

\subsection{3) Cultivation of Pseudomonas syringae pv. maculicola ES4326 (Psm)}

Psm was grown in Kings B media supplemented with rifampicin (50 mg/L). To maintain a fresh stock, the bacteria was subcultured onto new Kings B plates once every three weeks. Plates were incubated overnight $(\mathrm{O} / \mathrm{N})$ at $27^{\circ} \mathrm{C}$ and stored at $4^{\circ} \mathrm{C}$. For liquid cultures, a single colony was picked from plate and dropped into test tubes containing Kings media. The tubes were incubated at $27^{\circ} \mathrm{C}, 0 / \mathrm{N}$, shaking at $220 \mathrm{rpm}$. 
Table 10: Composition of Kings B media

\begin{tabular}{l|l}
\hline Component & Amount \\
\hline Protease Peptone & $10.0 \mathrm{~g}$ \\
\hline $\mathrm{K}_{2} \mathrm{HPO}_{4}$ & $1.5 \mathrm{~g}$ \\
\hline Glycerol & $1.0 \mathrm{~g}$ \\
\hline $\mathrm{dH}_{2} \mathrm{O}$ & to $1 \mathrm{~L}$ \\
\hline
\end{tabular}

pH was adjusted to 7.0 using $\mathrm{HCl}$.

The media was autoclaved and $5 \mathrm{ml}$ of sterile $1 \mathrm{M} \mathrm{MgSO}_{4}$ was added.

\subsection{4) Bacterial growth assay}

For bacterial growth assays with Psm, three leaf discs each from three infected leaves per plant were placed into tubes containing $200 \mu \mathrm{l}$ of $10 \mathrm{mM} \mathrm{MgCl}_{2}$ and a single $5 \mathrm{~mm}$ metal bead. The leaves were homogenized by placing the tubes in a paint mixer and shaking for three mins. Dilutions from $10^{-1}$ to $10^{-3}$ were made and $30 \mu \mathrm{l}$ of each dilution was plated on Kings B plates. The plates were incubated at $29^{\circ} \mathrm{C}$, and the colonies were counted after 2 days. A minimum of six biological replicates were used for each experiment. The number of Colony forming units (CFU) was normalized to the leaf area and was calculated based on the following formula:

$$
\mathrm{CFU}=\frac{\mathrm{N}(\mathrm{CFU}) * \text { dilution factor }}{\mathrm{A} * \mathrm{~V}}
$$

$\mathrm{N}$ : Number of colonies; A: Area of 9 leaf discs; V: Volume

\subsection{5) Pharmacological treatment with SA or NHP}

Pharmacological treatment with SA or NHP was performed by infiltrating three leaves of 4.5-week-old plants with $1 \mathrm{mM}$ sodium salicylate (SA) or $1 \mathrm{mM} \mathrm{N}$ hydroxy pipecolic acid (NHP) which was diluted in $10 \mathrm{mM} \mathrm{MgCl} 2$ solution. 
Samples were collected at the indicated time points. The NHP used in this thesis was generously donated by the Zeier lab (Hartmann et al., 2018). For priming experiments, three lower leaves were infiltrated with Psm (O.D. 0.005), two days after primary infection, the upper leaves were infiltrated with $1 \mathrm{mM}$ NHP. The upper leaves were collected after 8 hours for gene expression analysis.

\subsection{6) Botrytis cinerea infection assays}

B. cinerea strain B.05.10 spores, provided by Dr. Marcel Wiermer (University of Goettingen), was diluted to $5 \times 10^{4}$ spores $\mathrm{ml}^{-1}$ in potato dextrose broth. For growth assays, droplets of $5 \mu \mathrm{l}$ of spore suspension were placed on leaves of 6-week-old plants. The lesion diameter was measured after 3 days. For gene expression analysis, 4.5-weekold plants were sprayed with a $B$. cinerea spore solution $\left(2 \times 10^{5}\right.$ spores $\left.\mathrm{ml}^{-1}\right)$ or with quarter-strength potato dextrose broth (Mock) and the samples were collected 2 days post spraying.

Table 11: Composition of potato dextrose broth

\begin{tabular}{l|l}
\hline Component & Amount \\
\hline Potato Dextrose Broth & $12 \mathrm{~g}$ \\
\hline $\mathrm{dH}_{2} \mathrm{O}$ & $1 \mathrm{~L}$ \\
\hline
\end{tabular}

\subsection{7) Cultivation of Escherichia coli}

Luria-Bertani (LB) plates or LB/dYT broth supplemented with the required antibiotics were used for cultivating E.coli. For growth in solid media, streaked plates were incubated $0 / \mathrm{N}$ at $37^{\circ} \mathrm{C}$ and stored at $4^{\circ} \mathrm{C}$. For growth in liquid cultures, flasks or test tubes were used, which were incubated at $37^{\circ} \mathrm{C}$, shaking at $220 \mathrm{rpm}$. 
Table 12: Composition of LB medium

\begin{tabular}{l|l}
\hline Component & Concentration \\
\hline Yeast extract & $0,5 \%(\mathrm{~g} / \mathrm{l})$ \\
\hline Tryptone/peptone from casein & $1 \%(\mathrm{~g} / \mathrm{l})$ \\
\hline $\mathrm{NaCl}$ & $1 \%(\mathrm{~g} / \mathrm{l})$ \\
\hline
\end{tabular}

Table 13: Composition of dYT medium

\begin{tabular}{l|l}
\hline Component & Concentration \\
\hline Yeast extract & $1 \%(\mathrm{~g} / \mathrm{l})$ \\
\hline Tryptone/peptone from casein & $1.6 \%(\mathrm{~g} / \mathrm{l})$ \\
\hline $\mathrm{NaCl}$ & $0.5 \%(\mathrm{w} / \mathrm{v})$ \\
\hline
\end{tabular}

The $\mathrm{pH}$ was adjusted to 7.2 using $1 \mathrm{mM} \mathrm{NaOH}$. The media was autoclaved

\begin{tabular}{l|l|l}
\hline Antibiotic & Stock concentration & Working concentration \\
\hline Ampicillin & $100 \mathrm{mg} / \mathrm{ml}$ & 100 to $200 \mu \mathrm{g} / \mathrm{ml}$ \\
\hline Gentamicin & $25 \mathrm{mg} / \mathrm{ml}$ & $25 \mu \mathrm{g} / \mathrm{ml}$ \\
\hline Spectinomycin & $50 \mathrm{mg} / \mathrm{ml}$ & $100 \mu \mathrm{g} / \mathrm{ml}$ \\
\hline
\end{tabular}

Table 14: Antibiotic Concentrations used for E.coli transformation 


\subsection{8) Recombination in DH10 $\alpha$ BacY (Protocol from Cramer Lab - MPI}

\section{Biophysical Chemistry, Göttingen)}

To express recombinant NPR1 protein in insect cells, a Baculovirus Expression System was used. The methods described here are taken from a protocol provided by the Cramer Lab - MPI Biophyical Chemistry, Göttingen. The experiments involving expression of NPR1 in insect cells was performed by Pascal Mrozek (AG Gatz).

The 438-series vector containing strep-Maltose Binding Protein (MBP)-NPR1 under the control of polyhedrin (polh) promoter (see 5.2.1) was electroporated into E. coli $\mathrm{DH} 10 \alpha \mathrm{BacY}$ cells. The DH10 $\alpha \mathrm{BacY}$ cells contains Autographa californica multinucleocaspid nucleopolyhedrovirus (AcMNPV) bacmid engineered from a baculovirus genome, and a helper plasmid encoding the Tn7 transposase. The AcMNPV contains a YELLOW FLOURESCENCE PROTEIN (YFP) gene under the control of the polh promoter. The transformed cells were grown for 4 hours to facilitate recombination and streaked on agar plates containing 1 $\mathrm{mM}$ isopropyl beta-D-1-thiogalactopyranoside (IPTG), gentamycin and 150 $\mu \mathrm{g} / \mathrm{mL}$ 5-bromo-4-chloro-3-indolyl- $\beta$-D-galactopyranoside (X-Gal). Integration of the gene of interest into the bacmid disrupts the LacZ gene, resulting in white colonies for successful recombination. To confirm positive selection, the picked white colonies were streaked again on plates containing gentamycin, IPTG, and $\mathrm{X}$-Gal. The positive clones were then grown in $5 \mathrm{~mL} \mathrm{LB}$ cultures under gentamycin selection. Bacmid DNA was isolated from E. coli by alkaline lysis followed by isopropanol precipitation.

\subsection{9) Isolation of bacmid DNA by alkaline lysis/ isopropanol /ethanol precipitation}

For the isolation of bacmid DNA, the entire $5 \mathrm{ml}$ culture mentioned in the previous section was centrifuged at $4000 \mathrm{rpm}$ at $20^{\circ} \mathrm{C}$ for $15 \mathrm{~min}$. The supernatant was decanted and the pellet was resuspended in $250 \mu \mathrm{L}$ 
resuspension buffer (P1) from commercially available plasmid isolation kit. The resuspended pellet was then transferred to a $2 \mathrm{~mL}$ tube. $250 \mu \mathrm{L}$ of lysis buffer (P2) was added and inverted 3-5 times. Subsequently, 350 $\mu \mathrm{L}$ of neutralization buffer (N3) was added and the tubes were inverted 3-5 times. The lysed cells were centrifuged at $15000 \mathrm{rpm}$ for $10 \mathrm{~min}$ in a table-top centrifuge at $4^{\circ} \mathrm{C}$, and the supernatant was transferred to fresh $2 \mathrm{ml}$ tube. To precipitate the DNA from collected supernatant, $700 \mu \mathrm{L}$ isopropanol was added and inverted 3-5 times, the tubes were then incubated at $-20^{\circ} \mathrm{C} 0 / \mathrm{N}$. The next day, tubes were centrifuged at $15000 \mathrm{rpm}$ for $30 \mathrm{~min}$ at $4^{\circ} \mathrm{C}$ in a table-top centrifuge. The supernatant was carefully removed and $500 \mu \mathrm{L} 70 \%$ ethanol was added. The tubes were centrifuged at $15000 \mathrm{rpm}$ for $10 \mathrm{~min}$ at $4^{\circ} \mathrm{C}$ and the ethanol was decanted. $30-$ $200 \mu \mathrm{L} 70 \%$ ethanol was added to cover the DNA pellet, and stored at $-20^{\circ} \mathrm{C}$ until use.

Table 15: Composition of buffers used for alkaline lysis

\begin{tabular}{l|l|l}
\hline $\begin{array}{l}\text { Cell resuspension buffer } \\
\text { (P1) }\end{array}$ & $\begin{array}{l}\text { Lysis solution } \\
\text { (P2) }\end{array}$ & $\begin{array}{l}\text { Neutralization solution } \\
\text { (N3) }\end{array}$ \\
\hline $50 \mathrm{mM}$ Tris pH 8.0 & $0.2 \mathrm{M} \mathrm{NaOH}$ & $4 \mathrm{M} \mathrm{KOAc}, \mathrm{pH}$ adjusted \\
$50 \mathrm{mM}$ Glucose & $1 \% \mathrm{SDS}$ & to 5.5 with HCL \\
$10 \mathrm{mM}$ EDTA & & \\
$0.1 \mathrm{mg} / \mathrm{ml}$ DNAse free & & \\
RNAse A & & \\
\hline
\end{tabular}

\subsubsection{0) Insect cell lines used for bacmid transfection}

To get an appropriate amount of protein, insect cells were transfected with the bacmid containing the gene of interest. Once the insect cells take up the bacmid, the baculovirus DNA including the gene of interest is integrated in the insect genome.

Sf9 and Hi5 insect cell lines were used for transfection. Sf9 originates from pupal ovaries of the Fall Army Worm (Spodoptera frugiperda), whereas the Hi5 cell line was isolated from cabbage looper (Trichoplusia ni) ovarian cells. 


\subsubsection{1) Cultivation of Baculovirus}

\section{V0 virus production}

Two independent preparations of bacmid isolates for each construct were used for transfection. The ethanol covering the bacmid DNA was decanted and the DNA was allowed to dry by leaving the tubes open inside the hood for $10 \mathrm{~min}$. $20 \mu \mathrm{L}$ water was added to the pellet and incubated for $20 \mathrm{~min}$ until the pellets were fully dissolved. Once the pellets dissolved, $200 \mu \mathrm{L}$ serum free medium (SFM) (Gibcoß, Sf- $900^{\mathrm{TM}}$, from ThermoFisher) and $100 \mu \mathrm{L}$ of master mix - consisting of $10 \mu \mathrm{L}$ Xtreme Gene 9 transfection agent (Roche) and $100 \mu \mathrm{L}$ SFM medium was added for each bacmid transfection. The samples were incubated for $60 \mathrm{~min}$ inside the hood. After the incubation period, $150 \mu \mathrm{L}$ of transfection/DNA mix was added to 6 well plates containing $3 \mathrm{ml}$ of Sf9 cells at a concentration of $1 \mathrm{E}^{6}$ cells $/ \mathrm{mL}$ (Cell counted using CASY® cell counter, which assess cell viability based on the integrity of plasma membrane. A viable cell does not allow current to pass through, while current can pass through dead cells. The CASY® counter aligns the cells to a precision measuring pore, which passes exposes cells to an electric field. The device gathers information regarding the cell volume and viability).

The plates were incubated at $27^{\circ} \mathrm{C}$ for two to three days, and visualized under a fluorescent microscope. At this stage, YFP will be expressed in successfully transfected cells, which is an indication of the production of viral particles. The YFP fluorescence being visible in small areas expanded over time. On the $3^{\text {rd }}$ day, media above the cells containing recombinant viral particles was collected and transferred to a $15 \mathrm{ml}$ falcon tube. The supernatant obtained was labelled as V0 and stored at $4^{\circ} \mathrm{C}$ until further use.

\section{V1 virus production}

$150 \mu \mathrm{L}-3 \mathrm{~mL}$ of $\mathrm{V} 0$ virus was added to $25 \mathrm{ml}$ of Sf9 cells at a concentration of 1 $\mathrm{E}^{6}$ cells/mL. The cells divide once within 24 hours of adding the virus. The concentration of the cells were maintained at $1 \mathrm{E}^{6}$ cells $/ \mathrm{mL}$ by adding SFM medium. Once the cells stopped dividing, it was grown for an additional 48-72 
hours. The cell viability was monitored daily using the CASY ${ }^{\circledR}$ counter. Once the cell viability reached $\sim 80-88 \%$, cells were harvested by transferring the cell suspension into a sterile $50 \mathrm{ml}$ tube. The tubes were centrifuged, and the supernatant was transferred into a fresh $50 \mathrm{ml}$ tube and labelled as V1 samples.

\section{V2 virus production}

For V2 virus production, $200 \mu \mathrm{l}-2 \mathrm{~mL}$ of V1 virus was added to $600 \mathrm{ml}$ of Hi5 cells, freshly diluted to a concentration of $1 \mathrm{E}^{6}$ cells $/ \mathrm{mL}$ in a fresh $3 \mathrm{~L}$ flask. Like the $\mathrm{V} 1$ virus production, the cells were maintained at a concentration of $1 \mathrm{E}^{6}$ cells/ml. Once the cells stopped dividing, they were additionally grown for another 3 days and collected when viability reached $\sim 80-88 \%$. For harvesting the cells, samples were transferred to centrifuge bottles and centrifuged at $238 \mathrm{~g}$ for 30 mins at $4^{\circ} \mathrm{C}$. The supernatant was decanted and the pellet was diluted in the required extraction buffer. The samples thus obtained were used for protein enrichment using the ÄKTA system (See 5.4.6).

\section{2) Work with DNA}

\subsection{1) Ligation-independent cloning (LIC)}

To express NPR1 protein in insect cells, NPR1 was cloned into a 438 series vector (see 5.1.8) using the LIC strategy developed by QB3 MACROLABS, UC Berkeley. This technique utilizes the 3'-exonuclease activity of T4 polymerase to create complementary 5'-overhangs in the vector and gene of interest; this is similar to the sticky ends generated upon treatment with restriction enzymes. The overlaps generated are long enough to allow annealing by simply mixing the two fragments at room temperature, therefore negating the use of restriction enzymes and a ligation step.

NPR1 was PCR amplified with primers to generate LIC sites (Primers used shown in table 6). The components used for the PCR reactions are shown in table 17, and the program used is shown in table 18. 
Table 17: PCR reaction for amplification of NPR1

\begin{tabular}{l|l}
\hline Component & Volume $(\mu \mathrm{l})$ \\
\hline 5X Buffer & 10 \\
\hline dNTP $(10 \mathrm{mM})$ & 1 \\
\hline DMSO & 2.5 \\
\hline Forward primer $(10 \mu \mathrm{M})$ & 2.5 \\
\hline Reverse primer $(10 \mu \mathrm{M})$ & 2.5 \\
\hline Phusion polymerase & 0.5 \\
\hline Template DNA & Volume corresponding to $100 \mathrm{ng}$ \\
\hline Final volume & $50 \mu \mathrm{l}$ \\
\hline
\end{tabular}

Table 18: PCR program used for amplification of NPR1

\begin{tabular}{l|c|c|c}
\hline Step & $\begin{array}{c}\text { Temperature } \\
{\left[{ }^{\circ} \mathbf{C}\right]}\end{array}$ & Duration [min] & $\begin{array}{c}\text { Number of } \\
\text { cycles }\end{array}$ \\
\hline $\begin{array}{l}\text { Initial } \\
\text { denaturation }\end{array}$ & 98 & 0.5 & 1 \\
\hline Denaturation & 98 & 0.25 & 35 \\
\hline Annealing & 60 & 0.5 & 35 \\
\hline Extension & 72 & $0.5 / \mathrm{kb}$ & 35 \\
\hline Final extension & 72 & 10 & 1 \\
\hline Hold & 4 & $\infty$ & 1 \\
\hline
\end{tabular}

The PCR product was run on $1 \%$ agarose gel, specific band was cut and extracted,

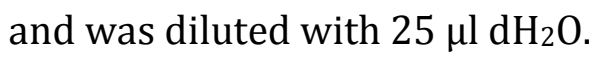

The LIC vector (438-series vector containing strep-Maltose Binding Protein [MBP] under the control of the polyhedrin [polh] promoter) was linearized using SspI. Components used for linearization of the vector is shown in table 19. 
Table 19: Linearization of 438C vector

\begin{tabular}{l|l}
\hline Component & Volume $(\mu \mathrm{l})$ \\
\hline 10X CutSmart Buffer & 5 \\
\hline SspI-HF $(10 \mathrm{U} / \mu \mathrm{L})$ & 2 \\
\hline DNA & Maximum $43 \mu \mathrm{l}(2 \mu \mathrm{g})$ \\
\hline Sterile dH20 & $\begin{array}{l}\text { Amount required for final volume of } \\
50 \mu \mathrm{l}\end{array}$ \\
\hline
\end{tabular}

The reaction was incubated at $37^{\circ} \mathrm{C}$ for four hours, purified after running on $1 \%$

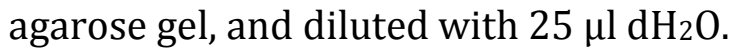

The linearized vector and gene of interest were then individually treated with T4 DNA polymerase in the presence of specific nucleotides (dGTP or dCTP). Components used in a LIC reaction are listed in table 20.

Table 20: LIC reaction

\begin{tabular}{l|l|l}
\hline Component & Volume $(\boldsymbol{\mu l})$ & $\begin{array}{l}\text { Final } \\
\text { concentration }\end{array}$ \\
\hline 5x Buffer for T4 DNA Polymerase & $4 \mu \mathrm{l}$ & $1 \mathrm{x}$ \\
\hline PCR product / Linearized vector & $\begin{array}{l}\text { Maximum } 14.6 \mu \mathrm{l}(150 \\
\mathrm{ng})\end{array}$ & \\
\hline DTT $(100 \mathrm{mM})$ & $1 \mu \mathrm{l}$ & $5 \mathrm{mM}$ \\
\hline dNTP $(25 \mathrm{mM})^{*}$ & $2 \mu \mathrm{l}$ & $2.5 \mathrm{mM}$ \\
\hline T4 DNA Polymerase $(5 \mathrm{U} / \mu \mathrm{l})$ & $0.4 \mu \mathrm{l}$ & $0.1 \mathrm{U} / \mu \mathrm{l}$ \\
\hline
\end{tabular}

Sterile dH2O required for final volume of $20 \mu \mathrm{l}$ was added

*dGTP was used for vector and dCTP was used for insert.

The components were mixed in a PCR tube and the reaction was incubated at $25^{\circ} \mathrm{C}$ for $40 \mathrm{~min}$. The T4 polymerase was heat inactivated by treating at $75^{\circ} \mathrm{C}$ for 10 min. For LIC annealing, $2 \mu \mathrm{l}$ of vector and $2 \mu \mathrm{l} \mathrm{PCR} \mathrm{product} \mathrm{from} \mathrm{T4} \mathrm{reaction}$ were mixed and incubated for $10 \mathrm{~min}$ at RT. After incubation, this mixture was transformed in E. coli. DH10 $\alpha$ EMBacY. 


\section{3) Work with RNA}

\subsection{1) RNA extraction from A. thaliana}

For RNA extraction, three leaves per plant were collected in screwcap tubes containing $5 \mathrm{~mm}$ metal beads and immediately frozen in liquid nitrogen. The samples were placed on a pre-cooled adapter which was then placed in a tissue lyser (Retsch ${ }^{\circledR}$ ) for grinding.

Phenol-chloroform method was used for the extraction of RNA. $1 \mathrm{ml}$ of TRIZOL buffer was added to the tubes containing samples and vortexed for $10 \mathrm{~min}$. Next, $300 \mu \mathrm{l}$ chloroform was added and the tubes were vortexed again for $10 \mathrm{~min}$. The samples were centrifuged at $13000 \mathrm{rpm}$ for $30 \mathrm{~min}$ in a tabletop centrifuge at $4^{\circ} \mathrm{C}$. After centrifugation, $600 \mu \mathrm{l}$ of the supernatant was carefully transferred using a pipette to $2 \mathrm{ml}$ tubes containing $300 \mu \mathrm{l}$ of high salt buffer (HSP) and $300 \mu \mathrm{l}$ of isopropanol. The tubes were inverted several times and incubated at room temperature for $10 \mathrm{~min}$. The $2 \mathrm{ml}$ tubes containing samples were then centrifuged for at $13000 \mathrm{rpm} 45 \mathrm{~min}$ in a tabletop centrifuge at $4^{\circ} \mathrm{C}$. The supernatant was decanted after centrifugation, and $300 \mu \mathrm{l}$ of $70 \%$ ethanol was added to the pellets. The tubes were inverted several times followed by a quick centrifugation of 2 mins. The ethanol was removed, and a fresh batch of ethanol was pipetted into the tubes. The pellets, along with ethanol, were transferred to new $1.5 \mathrm{ml}$ tubes, after which the ethanol was removed using a $1 \mathrm{ml}$ pipette. The samples were allowed to dry briefly until the pellets turned translucent, and 100 $\mu \mathrm{l}$ distilled water was added. The samples were then heated at $65^{\circ} \mathrm{C}$ for $5 \mathrm{~min}$, followed by vortexing and centrifuging. The dissolved samples were stored at $20^{\circ} \mathrm{C}$. 
Table 21: Composition of TRIZOL buffer

\begin{tabular}{l|l}
\hline Component & Concentration \\
\hline Phenol saturated with 0.1 M citrate buffer & $38 \%(\mathrm{v} / \mathrm{v})$ \\
\hline Ammonium thiocyanate & $0.4 \mathrm{M}$ \\
\hline Guanidinium thiocyanate & $0.8 \mathrm{M}$ \\
\hline $3 \mathrm{M}$ Na-Acetat pH 5.2 & $3.3 \%(\mathrm{v} / \mathrm{v})$ \\
\hline $100 \%$ Glycerol & $5 \%(\mathrm{v} / \mathrm{v})$ \\
\hline
\end{tabular}

Table 16: Composition of HSP buffer

\begin{tabular}{l|l}
\hline Component & Concentration \\
\hline $\mathrm{NaCl}$ & $1.2 \mathrm{M}$ \\
\hline Tri-sodium citrate $\times 2 \mathrm{H}_{2} \mathrm{O}$ & $0.8 \mathrm{M}$ \\
\hline
\end{tabular}

\subsection{2) cDNA synthesis}

The RNA concentration was estimated using a NanoDrop 2000 spectrophotometer. For cDNA synthesis, the RNA samples were diluted to $1 \mu \mathrm{g}$ in

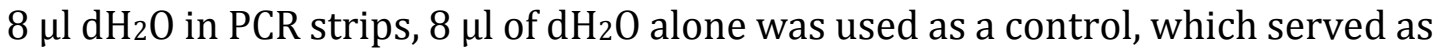
non-template control during Real time PCR.

The samples were first treated with DNase I. For this, a master mix (Table 23) consisting of DNase I and DNase buffer was prepared, $2 \mu \mathrm{l}$ was added to each sample and the strip was incubated for 30 mins at $37^{\circ} \mathrm{C}$ in a PCR cycler. Next, DNase I was inactivated by the addition of $1 \mu \mathrm{l}$ of $25 \mathrm{mM}$ EDTA, and incubating at $65^{\circ} \mathrm{C}$ for $10 \mathrm{~min}$.

Table 17: Master mix for DNase I treatment

\begin{tabular}{l|l}
\hline Component & Volume per reaction \\
\hline $10 x$ Reaction Buffer with $\mathrm{MgCl}_{2}$ & $1 \mu \mathrm{l}$ \\
\hline DNase I, $1 \mathrm{U} / \mu \mathrm{l}$ & $1 \mu \mathrm{l}$ \\
\hline
\end{tabular}


Then, oligo dT-primers were used to prime the cDNA synthesis. A master mix comprising of oligodT primers and water (Table 24) was prepared, and $1.2 \mu \mathrm{l}$ was added to each sample. The samples were heated at $70^{\circ} \mathrm{C}$ for $10 \mathrm{~min}$.

Table 18: Master mix for priming Oligo-dT primer binding

\begin{tabular}{l|l}
\hline Component & Volume per reaction \\
\hline Oligo dT-primer $(100 \mu \mathrm{M})$ & $0.2 \mu \mathrm{l}$ \\
\hline $\mathrm{d}_{2} \mathrm{O}$ & $1 \mu \mathrm{l}$ \\
\hline
\end{tabular}

Lastly, a third master mix consisting of the reaction buffer, dNTPs and reverse transcriptase was prepared (Table 25) and $7.8 \mu$ was added to each sample. The samples were heated at $42^{\circ} \mathrm{C}$ for $70 \mathrm{~min}$, and the reaction was stopped by heating at $70^{\circ} \mathrm{C}$ for $10 \mathrm{~min}$. The cDNA was stored at $-20^{\circ} \mathrm{C}$

Table 19: Master mix for cDNA synthesis

\begin{tabular}{l|l}
\hline Component & Volume per reaction \\
\hline $\mathrm{dH}_{2} \mathrm{O}$ & $1.5 \mu \mathrm{l}$ \\
\hline $5 \mathrm{x}$ Reaction Buffer for RT & $4 \mu \mathrm{l}$ \\
\hline $\mathrm{dNTPs}(10 \mathrm{mM})$ & $2 \mu \mathrm{l}$ \\
\hline Reverse Transcriptase $200 \mathrm{U} / \mu \mathrm{l}$ & $0.3 \mu \mathrm{l}$ \\
\hline
\end{tabular}

\subsection{3) Quantitative real-time PCR}

A 1:10 dilution of the cDNA was prepared in $\mathrm{dH}_{2} \mathrm{O}$ and $1 \mu \mathrm{l}$ of this dilution was used as a template during the qRT-PCR. A master mix consisting of reaction buffer, $50 \mathrm{mM} \mathrm{MgCl}_{2}$, primers, dNTPs, and polymerase (Table 26) was prepared. $24 \mu \mathrm{l}$ of the master mix was added to PCR strips and $1 \mu \mathrm{l}$ of the template cDNA was added to it. The strips were vortexed and spun in a micro centrifuge before transferring to the thermocycler. 
Table 20: Composition of qRT PCR reaction mix

\begin{tabular}{l|l|l}
\hline Component & Volume & Final concentration \\
\hline $\mathrm{dH}_{2} \mathrm{O}$ & $17.2 \mu \mathrm{l}$ & \\
\hline $10 \mathrm{x} \mathrm{NH}{ }_{4}$ Reaction Buffer & $2.5 \mu \mathrm{l}$ & $1 \mathrm{x}$ \\
\hline $50 \mathrm{mM} \mathrm{MgCl}_{2}$ & $1 \mu \mathrm{l}$ & $2 \mathrm{mM}$ \\
\hline $\mathrm{dNTPs}(10 \mathrm{mM})$ & $0.25 \mu \mathrm{l}$ & $100 \mu \mathrm{M}$ \\
\hline Primer mix $(4 \mu \mathrm{M}]$ & $2.5 \mu \mathrm{l}$ & $0.4 \mu \mathrm{M}$ \\
\hline SybrGreen $(1: 1000)$ & $0.25 \mu \mathrm{l}$ & $1: 100000$ \\
\hline Fluorescein $(1 \mu \mathrm{M})$ & $0.25 \mu \mathrm{l}$ & $10 \mathrm{nM}$ \\
\hline DNA Polymerase $5 \mathrm{U} / \mu \mathrm{l}$ & $0.05 \mu \mathrm{l}$ & $0.25 \mathrm{U}$ \\
\hline cDNA $(1: 10$ dilution) & $1 \mu \mathrm{l}$ & \\
\hline
\end{tabular}

The PCR strips were placed on the thermocyler and incubated according to the protocol shown in Table 27.

Table 21: PCR reaction sequence

\begin{tabular}{l|l|l|l}
\hline Step & Temp. $\left[{ }^{\circ} \mathbf{C}\right]$ & Duration [min] & Number of cycles \\
\hline $\begin{array}{l}\text { Initial } \\
\text { denaturation }\end{array}$ & 95 & 1.5 & 1 \\
\hline Denaturation & 95 & 0.20 & 39 \\
\hline Annealing & 55 & 0.20 & 39 \\
\hline Elongation & 72 & 0.40 & 39 \\
\hline Final Elongation & 72 & 4 & 1 \\
\hline Generation of & 95 & 1 & 1 \\
melting curve & 55 & 1 & 1 \\
\hline
\end{tabular}

The Ct values obtained were used to calculate the fold over reference using $2^{-\Delta \mathrm{Ct}}$ method (Schmittgen and Livak, 2008). UBQ5 was used as the reference gene.

$$
2^{-\Delta \mathrm{Ct}}=2^{-[\mathrm{CT}(\text { gene of interest })-\mathrm{CT}(\text { reference gene })]}
$$




\section{4) Work with proteins}

\subsection{1) Preparation of protein extracts from $A$. thaliana in urea buffer}

Plant materials were collected in screwcap tubes containing a stainless-steel bead ( $5 \mathrm{~mm})$ and immediately frozen. For grinding the samples, the tubes were transferred to precooled adaptors and ground using a tissue lyser (Retsch $®$ MM301). Approximately $100 \mathrm{mg}$ of the ground powder was mixed with $200 \mu \mathrm{l}$ urea buffer (Table 28) and heated for $10 \mathrm{~min}$ at $65^{\circ} \mathrm{C}$. The samples were centrifuged for $15 \mathrm{~min}$ at $10000 \mathrm{~g}$ in a tabletop centrifuge at RT, following which the supernatant was transferred to a new tube and stored at $-20^{\circ} \mathrm{C}$. For the estimation of protein concentration, Pierce 660 assay using the ionic detergent compatibility reagent (IDCR) was performed.

Table 22: Composition of Urea buffer

\begin{tabular}{l|l}
\hline Component & Concentration \\
\hline Urea & $4 \mathrm{M}$ \\
\hline SDS & $5 \%(\mathrm{w} / \mathrm{v})$ \\
\hline Glycerol & $16.7 \%(\mathrm{v} / \mathrm{v})$ \\
\hline Bromophenol blue & $0.06 \mathrm{~g} / \mathrm{l}$ \\
\hline
\end{tabular}

\subsection{2) Quantification of protein concentrations}

Pierce $® 660$ reagent was used for the quantification of protein. For the preparation of standard curve, $0,1,3,6$, and $9 \mu \mathrm{g}$ bovine serum albumin (BSA)

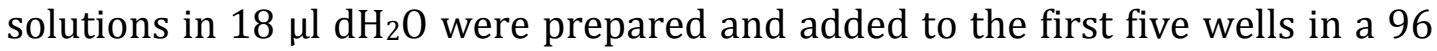

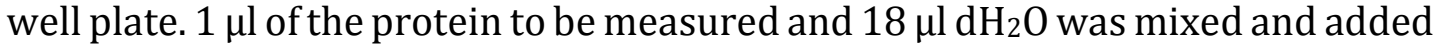
to a separate well. A mixture containing $150 \mu$ l Pierce ${ }^{\circledR} 660$ reagent and ionic detergent compatibility reagent (IDCR) $(50 \mathrm{mg} / \mathrm{ml}$ of reagent) was added to all the wells. The plate was incubated for $5 \mathrm{~min}$ at RT and A660nm was measured in a plate reader (Synergy HT). The protein concentration was estimated by using the standard curve obtained with BSA. 


\subsection{3) Polyacrylamide gel electrophoresis}

Mini-PROTEAN@ Tetra Cell System (1.5 mm thickness) was used to cast gels. The resolving gel solution was prepared with components shown in Table 29. APS and TEMED was added right before pouring the solution into the gel cast. To obtain an even surface, $100 \%$ isopropanol was added to the surface of the gel and a gel comb was pressed into position. Once the resolving gel solidified, the comb was removed, isopropanol was decanted, and the stacking gel solution (Table 30) was poured on top of the resolving gel. The comb was placed back into position to create the wells.

Table 29: Composition of $10 \%$ resolving gel

\begin{tabular}{l|l}
\hline Components & Volume[ml] \\
\hline $\mathrm{dH}_{2} \mathrm{O}$ & 4 \\
\hline $30 \%$ Acrylamide solution $(37.5: 1)$ & 3.3 \\
\hline $1.5 \mathrm{M}$ TRIS pH 8.8 & 2.5 \\
\hline $20 \%(\mathrm{w} / \mathrm{v}) \mathrm{SDS}$ & 0.05 \\
\hline $10 \%(\mathrm{w} / \mathrm{v}) \mathrm{APS}$ & 0.1 \\
\hline TEMED & 0.01 \\
\hline TOTAL VOLUME & 10 \\
\hline
\end{tabular}

Table 30: Composition of 4\% Stacking gel

\begin{tabular}{l|l}
\hline Components & Volume [ml] \\
\hline $\mathrm{dH}_{2} \mathrm{O}$ & 7.2 \\
\hline $30 \%$ Acrylamide solution & 1.34 \\
\hline $1.5 \mathrm{M}$ TRIS pH 6.8 & 1.25 \\
\hline $20 \%(\mathrm{w} / \mathrm{v}) \mathrm{SDS}$ & 0.05 \\
\hline $10 \%(\mathrm{w} / \mathrm{v})$ APS & 0.1 \\
\hline TEMED & 0.01 \\
\hline TOTAL VOLUME & 10 \\
\hline
\end{tabular}

During electrophoresis, the gels were assembled on the Mini-PROTEAN ${ }^{\circledR}$ Tetra Companion running module, placed in the electrophoresis chamber filled with $1 \mathrm{x}$ 
SDS buffer (Table 31), and the combs were removed. The protein samples were diluted in urea buffer, boiled for 10 minutes, spun down and loaded into the wells. Page Ruler ${ }^{\mathrm{TM}}$ prestained protein ladder was used as size standard. The gels were run at $80 \mathrm{~V}$ for $1.5 \mathrm{hrs}$.

Table 31: Composition of 10X SDS running buffer

\begin{tabular}{l|l}
\hline Component & Concentration \\
\hline Glycine & $1.9 \mathrm{M}$ \\
\hline SDS & $1 \%(\mathrm{w} / \mathrm{v})$ \\
\hline TRIS & $250 \mathrm{mM}$ \\
\hline
\end{tabular}

\subsection{4) Coomassie staining of polyacrylamide gels}

Gels to be stained were placed in a box containing coomassie staining solution and incubated at room temperature on a rocker overnight. The staining solution was decanted and destaining solution was added to the box, the gel was warmed in an oven at full power for approximately 2 minutes to accelerate the destaining process. The gels were scanned using an Epson Perfection V700 Photoscanner with the SilverFast-SE v6.6.1r2b software (LaserSoft Imaging).

Table 32: Composition of Coomassie Brilliant Blue (CBB) staining solution

\begin{tabular}{l|l}
\hline Component & Concentration \\
\hline Acetic acid & $10 \%(\mathrm{v} / \mathrm{v})$ \\
\hline Coomassie Brilliant Blue G250 & Half a spatula \\
\hline $\mathrm{Na}_{2} \mathrm{HPO}_{4} / \mathrm{NaH}_{2} \mathrm{PO}_{4} \mathrm{pH} 8.0$ & $50 \mathrm{mM}$ \\
\hline $\mathrm{NaCl}$ & $200 \mathrm{mM}$ \\
\hline
\end{tabular}

\subsection{5) Western blot analysis}

Samples separated by SDS-PAGE were blotted onto the polyvinyl difluoride (PVDF) membrane using a semi-dry method with constant current of $0.1 \mathrm{~mA} / \mathrm{cm}^{2}$ 
for 2 hours. After the transfer, the membrane was blocked with PBST (PBS, pH7.4; $0.1 \%$ Tween 20 ) containing $5 \%$ milk at room temperature for two hours and incubated overnight with the primary antibody (rabbit anti-NPR1 (AS121854, Agrisera)) at $4^{\circ} \mathrm{C}$. On the following day, the membrane was washed five times with PBST, and was incubated with the secondary antibody (horseradish peroxidase-conjugated anti-rabbit IgG (Agrisera)) for two hours at room temperature. Bands were detected using the Super SignalTM West Femto Maximum Sensitivity Substrate (Thermo Scientific).

\subsection{6) ÄKTA-based purification of strep-MBP-NPR1 from insect cells}

Strep-MBP-NPR1 was produced using the baculovirus system in Trichoplusia $n i$ (T. ni) cells by Pascal Mrotzek (AG Gatz) as described in section 5.1.8-5.1.11. A $600 \mathrm{ml}$ culture grown at $1 \mathrm{E}^{6}$ cells $/ \mathrm{ml}$ and a viability of approximately $88 \%$ was centrifuged at $238 \mathrm{~g}$ for $30 \mathrm{~min}$. The pellet obtained was resuspended in sodium phosphate lysis buffer, transferred to a $50 \mathrm{ml}$ tube and stored at $-80^{\circ} \mathrm{C}$. Prior to lysis, the cells were thawed by placing the tube in a beaker containing water. Once thawed; the tube was transferred to an icebox and sonicated using a Sonopuls sonifier with an M73 sonotrode at an amplitude of $30 \%$ and alternating a 0.4 secpulse and a $0.6 \mathrm{sec}$-break over $5 \mathrm{~min}$. The lysate was centrifuged at $20000 \mathrm{rpm}$ for 45 minutes at $4^{\circ} \mathrm{C}$ (Sorvall centrifuge RC6+, rotor SS-34). After centrifugation, the supernatant was filtered using a $0.45 \mu \mathrm{m}$ sterile filter. An aliquot of this sample was stored for use as crude extract control while running SDS PAGE.

Enrichment of Strep-MBP-NPR1 was performed using an ÄKTA prime plus purifier (GE Healthcare) connected to a $5 \mathrm{ml}$ MBPTrap column (GE Healthcare). First, the system was washed with degassed water, after which the column was equilibrated with 5 column volumes of sodium phosphate lysis buffer. The filtered crude extract was loaded onto the column at a flow rate of $0.5 \mathrm{ml} / \mathrm{min}$ using a $50 \mathrm{ml}$ superloop (GE Healthcare). The flow through was collected in $3 \mathrm{ml}$ fractions. After loading the crude extract, the column was washed with sodium phosphate lysis buffer until the baseline absorption at $280 \mathrm{~nm}$ of the lysis buffer 
was reached. The proteins bound to the column were eluted by washing the column with sodium phosphate buffer supplemented with $100 \mathrm{mM}$ maltose. Fractions were collected and stored at $4^{\circ} \mathrm{C}$ in a fridge. To analyze the quality of enrichment, fractions from each step was mixed with $2 x$ RPB and separated by SDS-PAGE.

Table 33: Composition of sodium phosphate lysis buffer

\begin{tabular}{|c|c|c|}
\hline Component & Amount & Concentration \\
\hline $\mathrm{Na}_{2} \mathrm{HPO}_{4}$ & $10.11 \mathrm{~g}$ & $37.7 \mathrm{mM}$ \\
\hline $\mathrm{NaH}_{2} \mathrm{PO}_{4}$ & $1.70 \mathrm{~g}$ & $12.3 \mathrm{mM}$ \\
\hline $\mathrm{NaCl}$ & $11.70 \mathrm{~g}$ & $200 \mathrm{mM}$ \\
\hline
\end{tabular}

Total volume of $1 \mathrm{~L}$ with $\mathrm{dH}_{2} \mathrm{O}$

$\mathrm{pH}$ was adjusted to 7.4 using $\mathrm{NaOH}$.

$100 \mathrm{mM}$ maltose was added to the lysis buffer, to prepare elution buffer.

Table 34: Composition of RPB buffer

\begin{tabular}{l|l}
\hline Component & Concentration \\
\hline TRIS pH 6.8 & $100 \mathrm{mM}$ \\
\hline SDS & $4 \%(\mathrm{~g} / \mathrm{l})$ \\
\hline Glycerol & $20 \%(\mathrm{~g} / \mathrm{l})$ \\
\hline Bromophenol blue & A spatula \\
\hline DTT & $200 \mathrm{mM}$ \\
\hline
\end{tabular}

\subsection{7) Protein dialysis}

Protein dialysis was performed using ZelluTrans dialysis tubing (MWCO: 12 - 14 $\mathrm{kDa}$, Roth). The tubings were soaked in $\mathrm{dH}_{2} \mathrm{O}$ for 10 mins, protein samples were added into the tubing and both ends were tied with a knot. NPR1 protein dialysis was performed in $500 \mathrm{ml}$ PBS buffer overnight, in the cold room with two buffer changes. The dialysis buffer was used for diluting samples used for isothermal titration calorimetry. 


\subsection{8) Trypsin digestion assay}

Strep-MBP-NPR1 protein was diluted down to $2 \mathrm{mg} / \mathrm{ml}$ in a Spin-X® UF 6 concentrator (MWCO $30 \mathrm{kDa}$ ) using reaction buffer containing $50 \mathrm{mM}$ Tris, $\mathrm{pH}$ 8.0, $200 \mathrm{mM} \mathrm{NaCl}, 1 \mathrm{mM}$ TCEP, and incubated with or without $1 \mathrm{mM}$ SA for 1 hour on ice. Trypsin was added to the sample protein at a final concentration of 0.005 $\mathrm{mg} / \mathrm{ml}$. The digestion reaction was carried out for 5,10 and $15 \mathrm{mins}$ at $20{ }^{\circ} \mathrm{C}$. After the digestion, $30 \mu \mathrm{g}$ of the digested protein was separated on $10 \%$ SDSPAGE and visualized by staining with Coomassie staining solution; the gels were destained and imaged using EPSON photo scanner.

\subsection{9) Isothermal titration calorimetry}

Isothermal titration calorimetry (ITC) was used to investigate the interaction between strep-MBP-NPR1 with SA. Strep-MBP-NPR1 was dialyzed twice for $12 \mathrm{~h}$ at $4{ }^{\circ} \mathrm{C}$ against $500 \mathrm{ml}$ PBS buffer, pH 7.4. The second dialysis buffer was used to dilute SA. The ITC cell was filled with $40 \mu \mathrm{M}$ Strep-MBP-NPR1 and the injection syringe was filled with $400 \mu \mathrm{M}$ of SA in PBS buffer $\mathrm{pH}$ 7.4. Overall, 19 injections were made with a speed of $2 \mathrm{~s} / \mu \mathrm{L}$ (first injection: $0.4 \mu \mathrm{L}$, injections 2-19: $2 \mu \mathrm{L}$ ) and a gap of 120 s. All experiments were carried out with a MicroCal PEAQ-ITC instrument (Malvern Panalytical) at $25^{\circ} \mathrm{C}$ with constant stirring of $750 \mathrm{rpm}$. The final amounts of heat per injection were fitted according to a 1:1 binding model.

Table 35: Composition of PBS buffer

\begin{tabular}{l|l|l}
\hline Reagent & Amount & Final concentration (1x) \\
\hline $\mathrm{NaCl}$ & $8 \mathrm{~g}$ & $137 \mathrm{mM}$ \\
\hline $\mathrm{KCl}$ & $0.2 \mathrm{~g}$ & $2.7 \mathrm{mM}$ \\
\hline $\mathrm{Na}_{2} \mathrm{HPO}_{4}$ & $1.44 \mathrm{~g}$ & $10 \mathrm{mM}$ \\
\hline $\mathrm{KH}_{2} \mathrm{PO}_{4}$ & $0.24 \mathrm{~g}$ & $1.8 \mathrm{mM}$ \\
\hline \multicolumn{2}{l}{ Total volume of 1 L with $\mathrm{dH}_{2} \mathrm{O}$}
\end{tabular}




\section{6) Results I - SA independent processes in SAR}

\section{1) The transcriptional regulation of FMO1 in Psm-infected SAR leaves is SA-independent, but NPR1-dependent.}

In a transcriptome analysis performed to study the induction of genes in uninfected SAR leaves, transcription of approximately $70 \%$ of the genes that were induced were found to be dependent on SA (Bernsdorff et al., 2016). This highlights the fact that SA is required for the establishment of a large part of the SAR response. FMO1 was amongst the genes which showed an SA-independent induction.

We were interested in studying the regulation of FMO1 in the context of SAR. Given that NPR1 is a critical component required for the actuation of the SAR response (Cao et al., 1994), we questioned if NPR1 plays a role in the SAindependent induction of FMO1.

To study the transcriptional regulation of FMO1 during SAR, the infection regime described by Bernsdorf et al., 2016 was followed. Briefly, the lower leaves of Col0, sid2, and $n p r 1$ plants were infiltrated with Psm (O.D. 0.005) or $\mathrm{MgCl}_{2}$, two days after which the secondary leaves were infiltrated with Psm (O.D. 0.005) and collected after 8 hours for analysis; hereafter referred to as mock-Psm and PsmPsm treatments. Gene expression was considered to be primed when genes were higher induced upon Psm-Psm treatment as compared to the mock-Psm treated plants.

When analyzing gene expression in mock-Psm-treated plants, we observed that the levels of FMO1 in the sid2 and npr1 mutants were lower than in the Col-0 plants. A different picture emerged after Psm-Psm treatment: here, induction of FMO1 was even elevated in the sid2 mutant, whereas the npr1 mutant did not show an induction, suggesting the presence of an SA-independent and NPR1dependent pathway that leads to the induction of FMO1. 
A

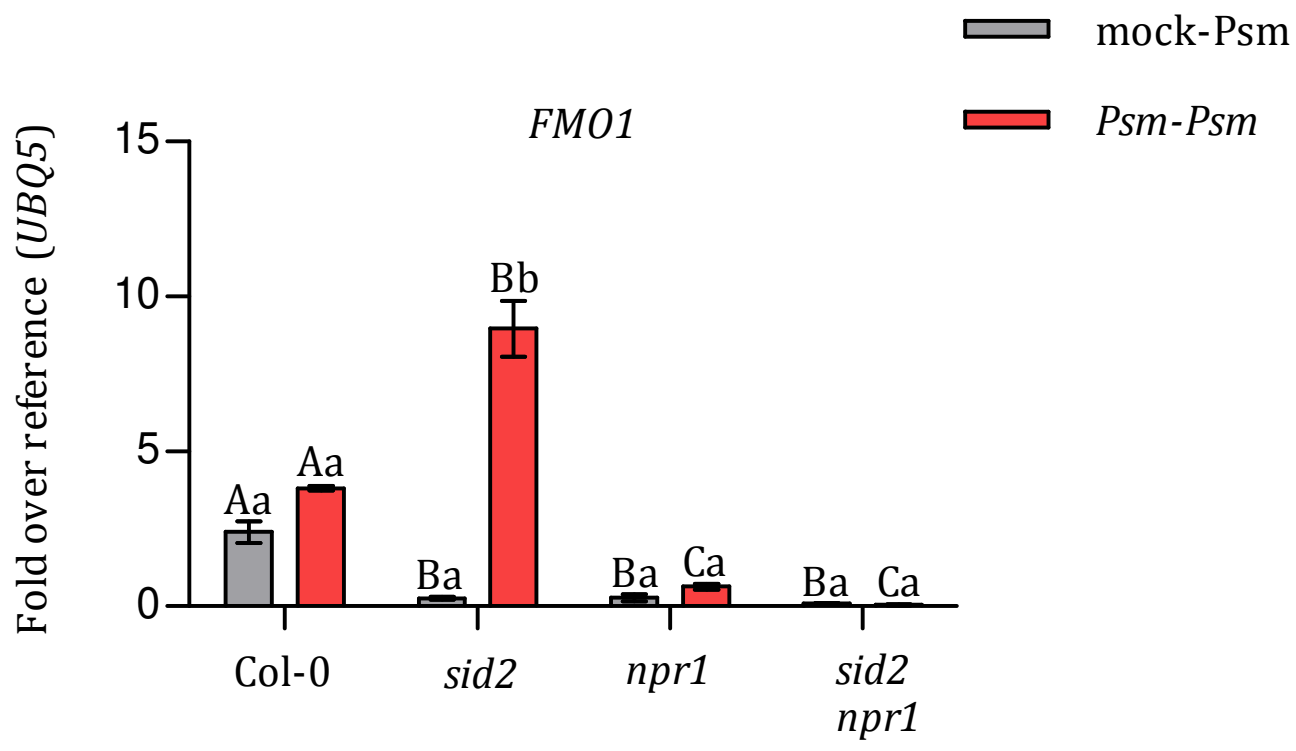

B

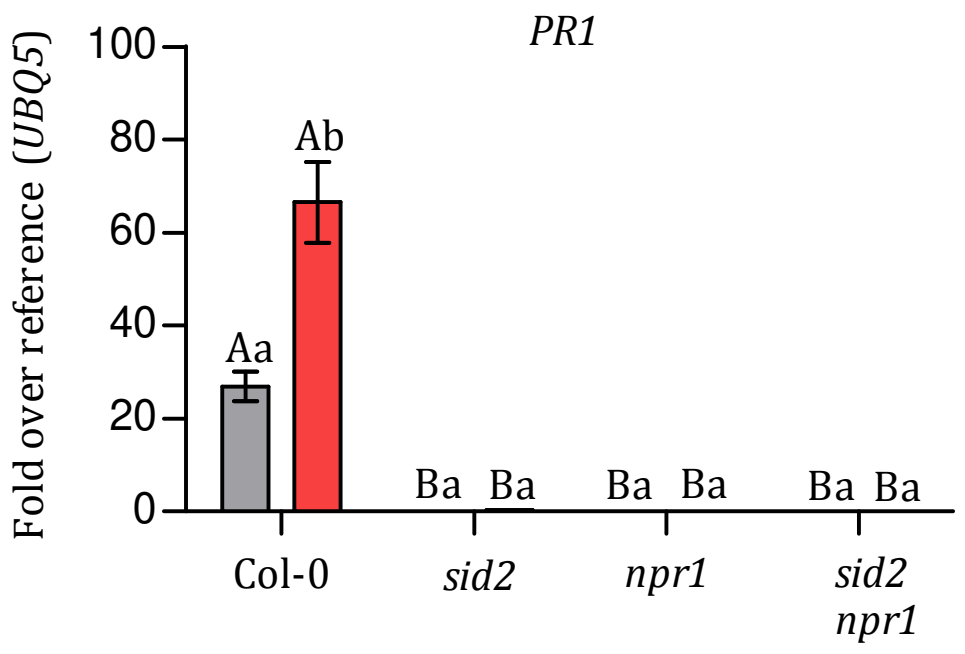

Figure 7: q-RT PCR analysis of FMO1 (A) and PR1 (B) in Col-0, sid2, npr1, and sid2 npr1 plants following mock-Psm and Psm-Psm treatments. The lower leaves of 4.5-week-old plants were infiltrated with $\mathrm{MgCl}_{2}$ (mock) or Psm (O.D. 0.005). Two days later, the systemic leaves were infiltrated with Psm (O.D. 0.005), and the systemic leaves were collected after 8 hours. Transcript levels were normalized to the transcript levels of $U B Q 5$. Error bars represent the average \pm SEM of three samples per genotype which were combined from three independent plants of the respective genotypes. Lowercase letters indicate significant differences $(P<0.05)$ between treatments performed with the same genotype; uppercase letters indicate significant differences $(P<0.05)$ between genotypes subjected to the same treatment. Statistical analysis was done using two-way ANOVA followed by Bonferroni's post-test. 
We observed that the transcript levels of $P R 1$, which is a classical SAR marker gene (Uknes et al., 1992), were low in the mock-Psm treated sid2 and npr1 mutants as compared to Col-0. Upon Psm-Psm treatment, the sid2 and npr1 mutants did not show an induction. Our results suggest that FMO1 and PR1 have different requirements concerning the NPR1-inducing metabolite leading to induction during SAR.

Next, we questioned whether the NPR1-dependent FMO1 induction seen in the Psm-Psm-treated sid2 mutant is due to a factor that comes only into action in the absence of SA. Given that SA can induce FMO1 in an NPR1-dependent manner (Supplemental Figure 1), the lack of FMO1 induction observed in the npr1 mutant could be due to it being a target of the SA signaling cascade. To scrutinize if the absence of FMO1 induction observed in the npr1 mutant is due to the role of NPR1 in the SA-dependent signaling cascade or if it is required for the SA-independent mechanism, we used the sid2 npr1 double mutant. The advantage of using this mutant is that SA biosynthesis is absent, making it possible to address the role of NPR1 in the SA-independent signaling cascade.

The levels of FMO1 in mock-Psm- and Psm-Psm- treated sid2 npr1 plants were lower as compared to Col-0 and showed levels similar to $n p r 1$ plants. As expected, the levels of PR1 in the sid2 npr1 mutant were identical to those in the sid2 and npr1 mutants following mock-Psm and Psm-Psm infections.

Our results confirm that NPR1 is essential for the SA-dependent signaling cascade leading to PR1 induction, as well as the SA-independent induction of FMO1 that is observed during Psm-Psm infections, but not during mock-Psm treatments.

Next, we speculated that the higher induction of FMO1 seen in the sid2 mutant upon Psm-Psm treatment may have to do with the accumulation of the NPR1 protein. In order to analyze the accumulation of NPR1, we extended the SAR assay by infiltrating the lower leaves of Col- 0 and sid2 plants with $\mathrm{MgCl}_{2}$ (mock) or Psm (O.D .0.005), two days after which the upper leaves were infiltrated with $\mathrm{MgCl}_{2}$ (Mock) or Psm (O.D. 0.005), leading to mock-mock, mock-Psm, Psm-mock, and 
Psm-Psm treatments. The upper leaves were collected 8 hours after infiltration for analysis.

\begin{tabular}{|c|c|c|c|c|c|c|c|c|}
\hline \multirow[b]{2}{*}{$\begin{array}{l}\text { Local } \\
\text { Systemic }\end{array}$} & \multicolumn{4}{|c|}{ Col-0 } & \multicolumn{4}{|c|}{ sid2 } \\
\hline & $\begin{array}{l}\text { mock } \\
\text { mock }\end{array}$ & $\begin{array}{l}\text { mock } \\
\text { Psm }\end{array}$ & $\begin{array}{l}\text { Psm } \\
\text { mock }\end{array}$ & $\begin{array}{l}\text { Psm } \\
\text { Psm }\end{array}$ & $\begin{array}{l}\text { mock } \\
\text { mock }\end{array}$ & $\begin{array}{l}\text { mock } \\
\text { Psm }\end{array}$ & $\begin{array}{l}\text { Psm } \\
\text { mock }\end{array}$ & $\begin{array}{l}\text { Psm } \\
\text { Psm }\end{array}$ \\
\hline
\end{tabular}

Figure 8: NPR1 protein levels in secondary leaves of Col-0 and sid2 plants. Lower leaves were infiltrated with mock $\left(\mathrm{MgCl}_{2}\right)$ or Psm (O.D. 0.005), two days after which the upper leaves were with mock or Psm (O.D. 0.005). Samples were collected after 8 hours for Western blot analysis using an antibody against NPR1. The bands correspond to the NPR1 protein $(66 \mathrm{kDa})$.

In Col-0 plants, an increase in NPR1 protein accumulation was observed following mock-Psm treatment. The protein levels also showed an increase after mock-Psm, Psm-mock and Psm-Psm treatments. The increase in the Psm-mocktreated samples shows that infiltrating the lower leaves of Col-0 with Psm results in the accumulation of NPR1 in the systemic leaves. The sid2 mutant revealed a similar pattern, with the NPR1 protein levels being somewhat lower in the extracts derived from mock-Psm-treated leaves. To relate these data to the gene expression pattern shown in Figure 1, the mock-Psm samples have to be compared to the Psm-Psm-treated samples, since FMO1 expression depends on SA in the first but not the latter case. This observation cannot be explained by alterations in NPR1 abundance. 
6.2) The conserved arginine residue (R432) in NPR1 is required for the induction of FMO1

In a recent work by Ding et al., the arginine (R432) residue, which is conserved in NPR1, NPR3 and NPR4, was shown to be essential for SA binding activity of recombinant NPR1 (Ding et al., 2018). Replacing the arginine residue by a glutamine impaired the ability of NPR1 to bind SA. Moreover, the npr1 mutant complemented with NPR1 harboring the arginine to glutamine mutation (NPR1 ${ }^{\text {R432Q) }}$ was impaired in the induction of genes following SA treatment. We were interested in addressing the role of the conserved arginine residue in the induction of FMO1 during SAR. To this end, we performed SAR experiments with npr1 complemented with wildtype NPR1 and complemented with the arginine mutated NPR1 (NPR1R4320). In contrast to what was observed in the experiment shown in Figure 7, FMO1 levels were low in mock-Psm-treated Col-0 plants. However, FMO1 was induced in the npr1 line transformed with wild type NPR1, while it was low in $n p r 1$, and NPR1 ${ }^{R 432 Q}$ plants. As previously reported, we observed induction of FMO1 in Col-0 after Psm-Psm treatment. The npr1 mutant complemented with wildtype NPR1 showed induction as well.

Contrary to previous experiments, an induction was observed in the Psm-Psmtreated npr1 mutant, a slight induction was also observed in the NPR1 ${ }^{\mathrm{R} 432 \mathrm{Q}}$ plants; albeit to levels lower than Col-0. The mock-Psm-treated plants showed a similar trend in terms of $P R 1$ induction, with low levels in all genotype except for the NPR1 plants. However, upon Psm-Psm treatment, PR1 was induced in Col-0 and NPR1 plants, while it was absent in the npr1 and NPR1 ${ }^{\text {R432Q }}$ plants. 


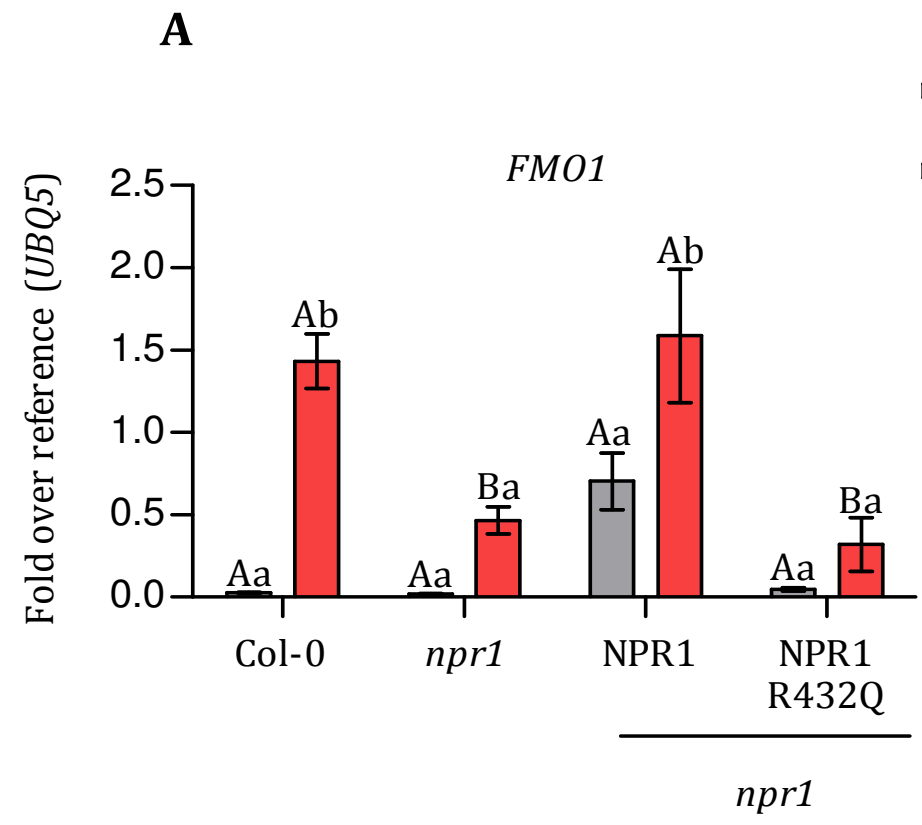

B

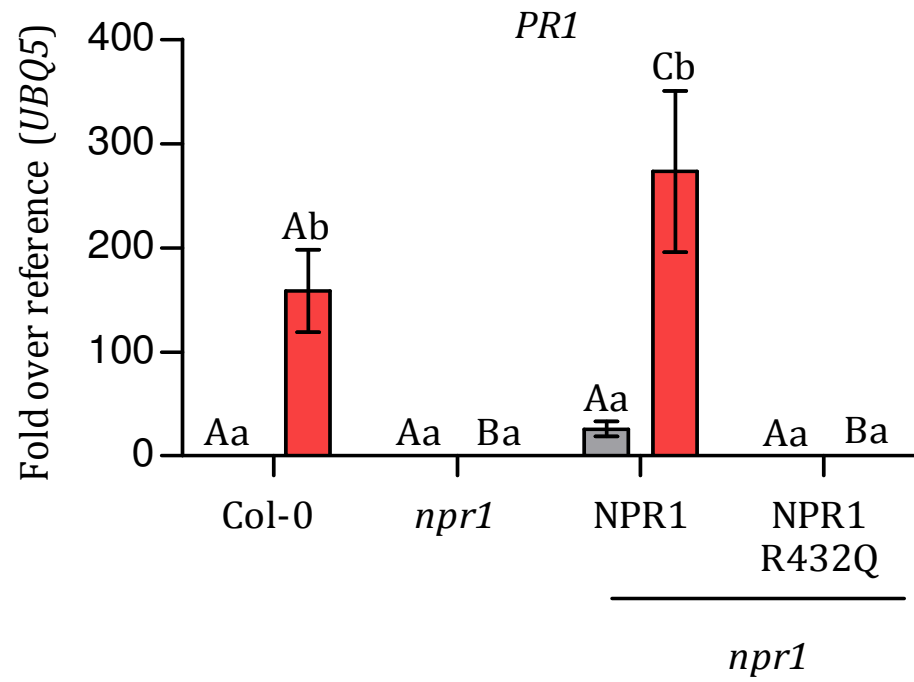

Figure 9: q-RT PCR analysis of $P R 1$ (A) and FMO1 (B) in Col-0, npr1, and npr1 plants complemented with NPR1 and NPR1 ${ }^{\text {R432Q }}$ following mock-Psm and Psm-Psm treatments. The lower leaves of 4.5-week-old plants were infiltrated with $\mathrm{MgCl}_{2}$ (mock) or Psm (O.D. 0.005). Two days later, the systemic leaves were infiltrated with Psm (O.D. 0.005 ), and the systemic leaves were collected after 8 hours. Transcript levels were normalized to the transcript levels of UBQ5. Error bars represent the average \pm SEM of four plants of each genotype. Lowercase letters indicate significant differences $(P<0.05)$ between treatments performed with the same genotype; uppercase letters indicate significant differences $(P<0.05)$ between genotypes subjected to the same treatment. Statistical analysis was done using two-way ANOVA followed by Bonferroni's post-test. 


\section{3) SA-independent FMO1 induction requires TGA2/5/6 and TGA1/4}

Given that our results point to a SA-independent and NPR1-dependent induction of FMO1, we next questioned as to how NPR1 contributes to the regulation of FMO1. NPR1 is a transcriptional coactivator that does not have a DNA binding domain but rather acts in concert with TGA transcription factors (Zhang et al., 1999).

To analyze the role of TGA transcription factors in the SA-independent induction of FMO1, tga 1 tga 4 and tga 2 tga 5 tga 6 mutants crossed into the sid2 background were used. We observed that FMO1 levels in the mock-Psm- treated sid2 tga2 tga5 tga6 and sid2 tga 1 tga 4 plants were as low as those observed in sid2 mutant. Following Psm-Psm treatment, the sid2 tga 2 tga 5 tga 6 and sid2 tga 1 tga 4 mutants did not show an induction of FMO1. The induction of PR1 was also hampered in the sid2 tga 2 tga 5 tga 6 and sid2 tga 1 tga 4 mutants.

It is concluded that even in the SA-independent pathway, NPR1-dependent transcriptional responses requires TGA factors. Whether they directly act at the FMO1 promoter or whether they act through the activation of transcription of other regulatory factors remains to be shown. 

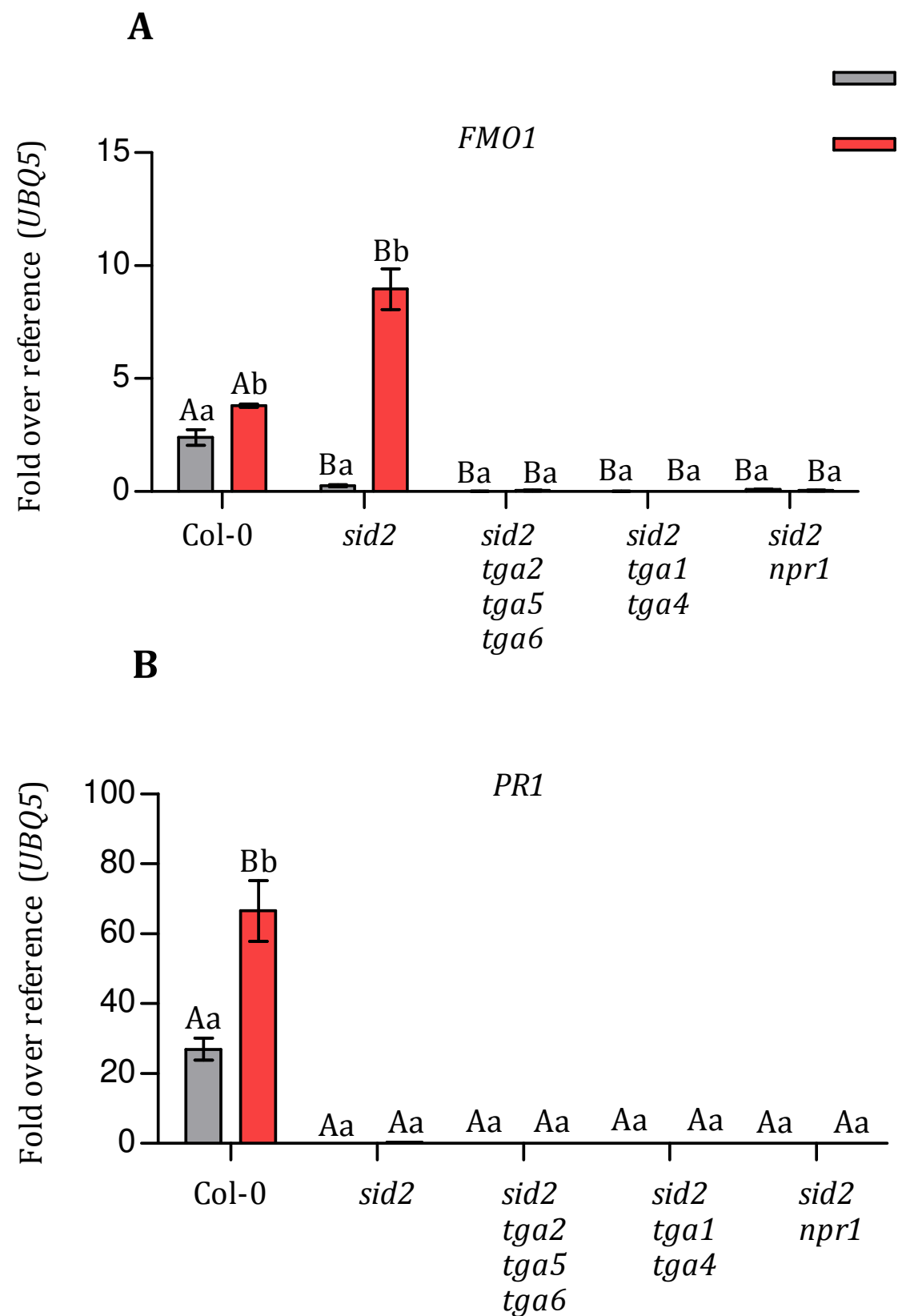

Figure 10: q-RT PCR analysis of FMO1 (A) and PR1 (B) in Col-0, sid2, sid2 tga256, sid2 tga14, and sid2 npr1 plants following mock-Psm and Psm-Psm treatments. The lower leaves of 4.5-week-old plants were infiltrated with $\mathrm{MgCl}_{2}$ or Psm (O.D. 0.005). Two days later, the systemic leaves were infiltrated with Psm (O.D. $0.005)$, and the systemic leaves were collected after 8 hours. Transcript levels were normalized to the transcript levels of $U B Q 5$. Error bars represent the average \pm SEM of three plants of each genotype. The experiment was performed once. Lowercase letters indicate significant differences $(P<0.05)$ between treatments performed with the same genotype; uppercase letters indicate significant differences $(P<0.05)$ between genotypes subjected to the same treatment. Statistical analysis was done using two-way ANOVA followed by Bonferroni's post-test. The data for Col-0, sid2, and sid2 npr 1 are the same as those presented in Figure 7. 


\section{4) SA-independent FMO1 induction requires ALD1}

We next questioned as to what the events upstream of NPR1, leading to the induction of FMO1, might be. Since NHP is important for SAR development and for expression of those genes, which were still induced in SAR leaves of the sid2 mutant (Bernsdorff et al., 2016; Hartmann et al., 2018), NHP was a likely candidate, and we decided to induce the SAR response in a system that lacks SA and NHP.

To this end, experiments were performed with sid2 ald1 plants, since we reasoned that the absence of a functional ALD1 would block the accumulation of Pipecolic acid and therefore lead to low amounts of NHP (Hartmann et al. 2018), thereby providing a condition where NPR1 is available, but SA and NHP are not.

In our experimental conditions, on comparing sid2 with sid2 ald1, we observed that following mock-Psm treatment, the FMO1 levels in sid2 ald1 mutant was similar to the sid2 and sid2 npr1 mutants. The enhanced expression observed in sid2 mutant upon Psm-Psm infections as compared to mock-Psm treatments were reproduced again, whereas the sid2 ald1 and sid2 npr1 mutants were deficient in the induction of FMO1. 


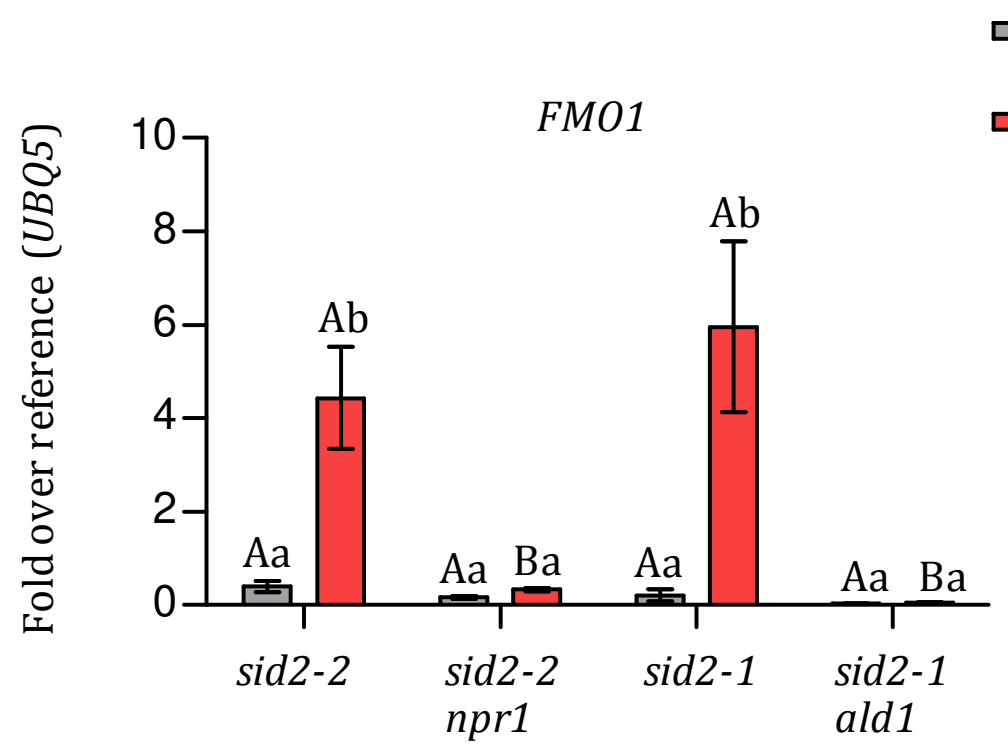

Figure 11: q-RT PCR analysis of FMO1 in sid2-2, sid2-2 npr1, sid2-1, and sid21 ald1 plants following mock-Psm and Psm-Psm treatments. The lower leaves of 4.5-week-old plants were infiltrated with $\mathrm{MgCl}_{2}$ (mock) or Psm (O.D. 0.005). Two days later, the systemic leaves were infiltrated with Psm (O.D. 0.005), and the systemic leaves were collected after 8 hours. Transcript levels were normalized to the transcript levels of UBQ5. Error bars represent the average \pm SEM of four plants of each genotype. Lowercase letters indicate significant differences $(P<0.05)$ between treatments performed with the same genotype; uppercase letters indicate significant differences $(P$ $<0.05$ ) between genotypes subjected to the same treatment. Statistical analysis was done using two-way ANOVA followed by Bonferroni's post-test.

Overall, our results suggest that in the absence of functional ALD1, the SAindependent and NPR1-dependent pathway leading to FMO1 expression is not operational. It is compelling to speculate that during SAR, a signaling cascade is initiated that requires SA along with its receptor NPR1 and transcription factors TGA2/5/6 and TGA1/4 for the induction of genes such as PR1, along with signaling events that involve NPR1 and TGA transcription factors without the requirement of SA but with NHP leading to the induction of genes such as FMO1. 
6.5) NHP treatment leads to SA synthesis which is required for full induction of FMO1 and $P R 1$

To confirm our hypothesis that NHP induces NPR1-dependent FMO1 expression, we performed pharmacological experiments using NHP. First, to verify that NHP treatment can lead to gene induction in the absence of SA, Col-0 and sid2 mutant plants were infiltrated with $1 \mathrm{mM} \mathrm{NHP}$, or $10 \mathrm{mM} \mathrm{MgCl}_{2}$ as mock treatment. Another set of plants was similarly infiltrated with $1 \mathrm{mM}$ SA to compare the efficiency of gene induction. As previous work by Chen et al. (2018) had shown that NHP treatment leads to FMO1 induction after 48 hours, we decided to collect samples after 8 and 48 hours to study the early and late responses.

Samples collected after 8 hours did not show FMO1 induction after treatment with NHP. However, in samples collected after 48 hours of treatment, an induction was observed in Col-0 plants. Although the NHP-treated sid2 mutant showed an increase in transcript levels after 48 hours, it was much less than in Col-0.

The induction of $P R 1$ followed a similar trend after NHP treatment, with Col-0 plants showing an induction at 48 hours after treatment. The levels of $P R 1$ in the NHP-treated sid2 mutant was several folds lower than Col-0. However, on comparing the PR1 transcript levels between the mock- and NHP-treated sid2 mutants, a slight induction is apparent in half of the NHP-treated plants (Figure $12 \mathrm{C}$ ). It is crucial to mention this trend here, as the slight SA-independent induction of PR1 by NHP becomes more evident in the section that follows (Figure 14 B). Finally, induction of ICS1 was observed in NHP-treated Col-0 plants. The predicted enhanced SA levels most likely serve to boost (Figure 12 D) FMO1 and $P R 1$ expression (Figure $12 \mathrm{D}$ ). This is different from the situation in Psm-infected SAR leaves (Figure 7), where SA is rather inhibitory for FMO1 induction. 
A

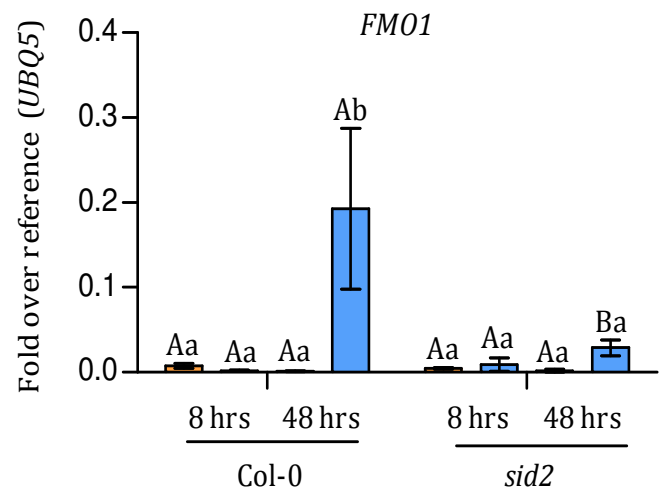

C

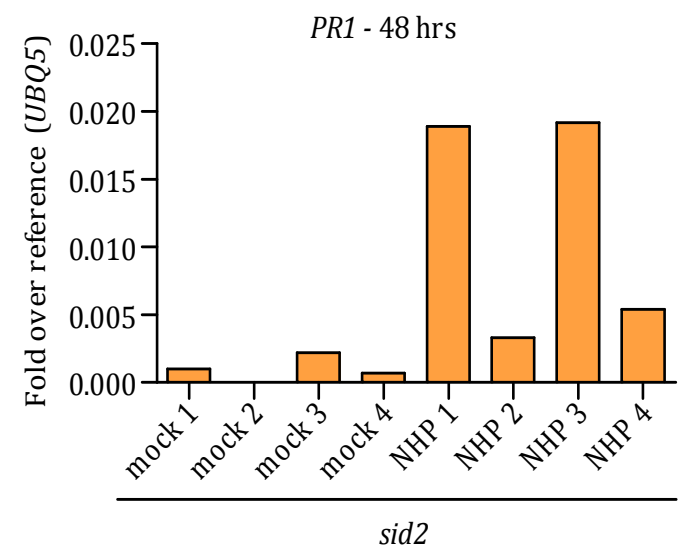

\section{B}

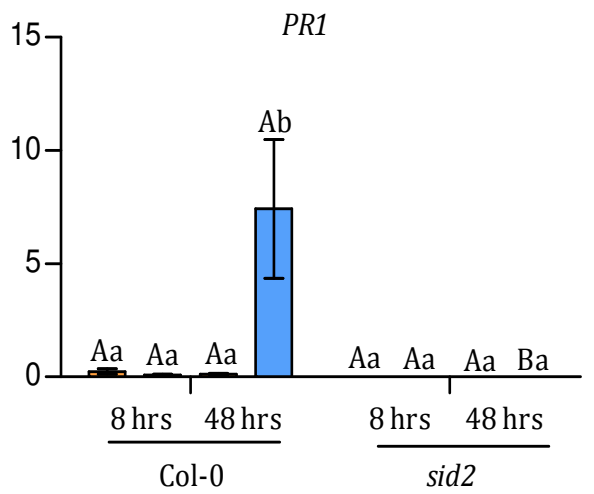

D

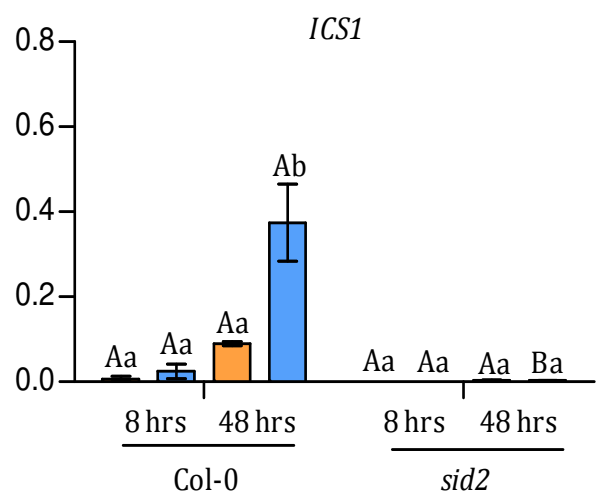

Figure 12: q-RT PCR analysis of FMO1 (A) PR1 (B) and ICS1 (D) in Col-0 and sid2 plants following mock and NHP treatments. (C) Transcript levels of PR1 in individual sid2 samples collected 48 hours after treatment. Three leaves of 4.5-week-old plants were infiltrated with $\mathrm{MgCl}_{2}$ (mock) or $1 \mathrm{mM}$ NHP. Leaves were collected after 8 and 48 hours. Transcript levels were normalized to the transcript levels of UBQ5. Error bars represent the average \pm SEM of four plants of each genotype. Lowercase letters indicate significant differences $(P<0.05)$ between treatments performed with the same genotype; uppercase letters indicate significant differences $(P$ $<0.05$ ) between genotypes subjected to the same treatment. Statistical analysis was done using two-way ANOVA followed by Bonferroni's post-test. 
To evaluate the efficiency of SA in inducing gene expression in a similar experimental setup, the transcript levels after SA treatment were compared to those after $\mathrm{MgCl}_{2}$ treatment. Upon infiltration with $1 \mathrm{mM} \mathrm{SA}$, a fivefold induction of FMO1 was observed in Col-0 and sid2 plants after 8 hours of treatment, whereas the samples collected after 48 hours of treatment did not show an induction. PR1 was induced to similar levels in Col-0 and sid2 mutants after 8 hours. The samples that were collected after 48 hours still showed increased transcript levels.

SA treatment led to the induction of ICS1 in Col-0 plants after 8 and 48 hours of treatment. Similar to the results shown in Figure $12 \mathrm{D}, \mathrm{MgCl}_{2}$ treatment led to elevated ICS1 transcript levels. However, the postulated increase in SA in these samples did not induce FMO1 or PR1.

A

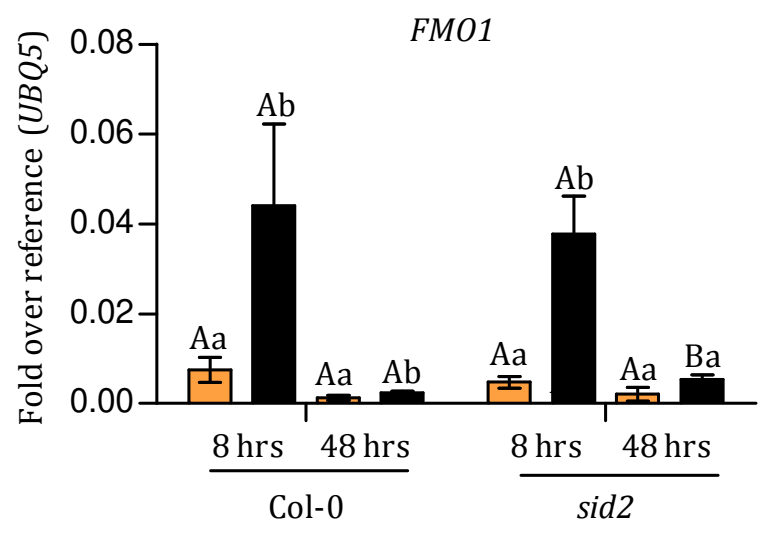

C

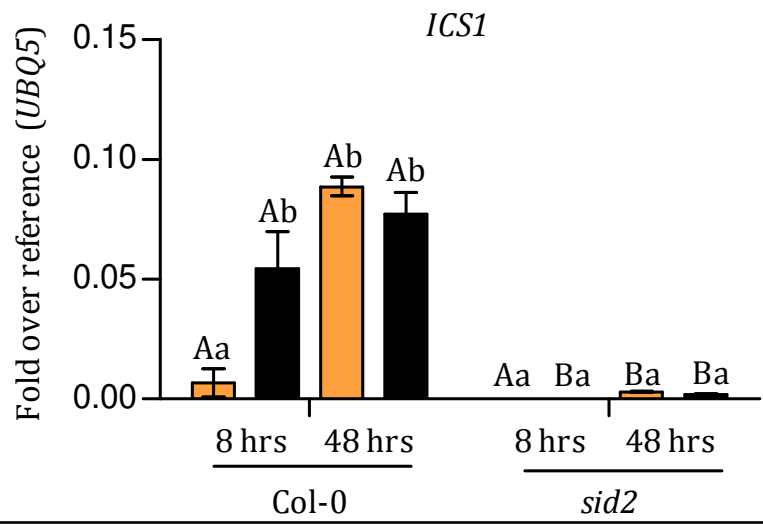

C
B

mock

SA

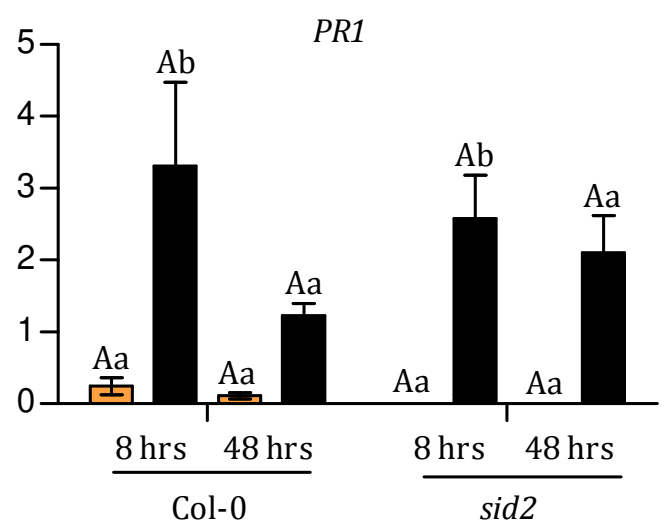


Figure 13: q-RT PCR analysis of FMO1 (A) PR1 (B) and ICS1(C) in Col-0 and sid2 plants following mock and SA treatments. Three leaves of 4.5-week-old plants were infiltrated with $\mathrm{MgCl}_{2}$ (mock) or $1 \mathrm{mM} \mathrm{SA}$. Leaves were collected after 8 and 48 hours. Transcript levels were normalized to the transcript levels of UBQ5. Error bars represent the average \pm SEM of four plants of each genotype. Lowercase letters indicate significant differences $(P<0.05)$ between treatments performed with the same genotype; uppercase letters indicate significant differences $(P<0.05)$ between genotypes subjected to the same treatment. Statistical analysis was done using two-way ANOVA followed by Bonferroni's post-test.

Based on the results from our pharmacological treatments, it is evident that SA and NHP follow different kinetics leading to gene induction. SA treatment results in the induction of genes within 8 hours, while NHP treatment showed prominent induction only in samples collected after 48 hours. Moreover, NHP is a weak inducer in the absence of SA as the induction of genes observed in sid2 following NHP treatment was very low.

Although the levels of FMO1 in the NHP treated sid2 plants were not significantly high in this batch of experiments, we observed higher levels of NHP-mediated induction of FMO1 and PR1 in the sid2 mutant in other experiments. This will be discussed in the sections that follow.

\section{6) NPR1 and TGA1/4 are required for the induction of FMO1 in NHP-treated plants}

On establishing that SA and NHP treatments lead to the induction of FMO1 and PR1, the functional significance of TGA1/4 and NPR1 in these signaling cascades was analyzed by utilizing the sid2 npr1 and the sid2 tga 1 tga 4 mutants. Given the fact that we are interested in the SA-independent processes, we decided to exclude Col-0 from this batch of experiment and compare the results to the sid2 genotype. Based on the previous experiments, we have established that SA 
induces genes 8 hours after induction, while the NHP-mediated induction is prominent at 48 hours after treatment.

For clarity of discussion, the results here concentrate on the samples treated with NHP for 48 hours and with SA for 8 hours.
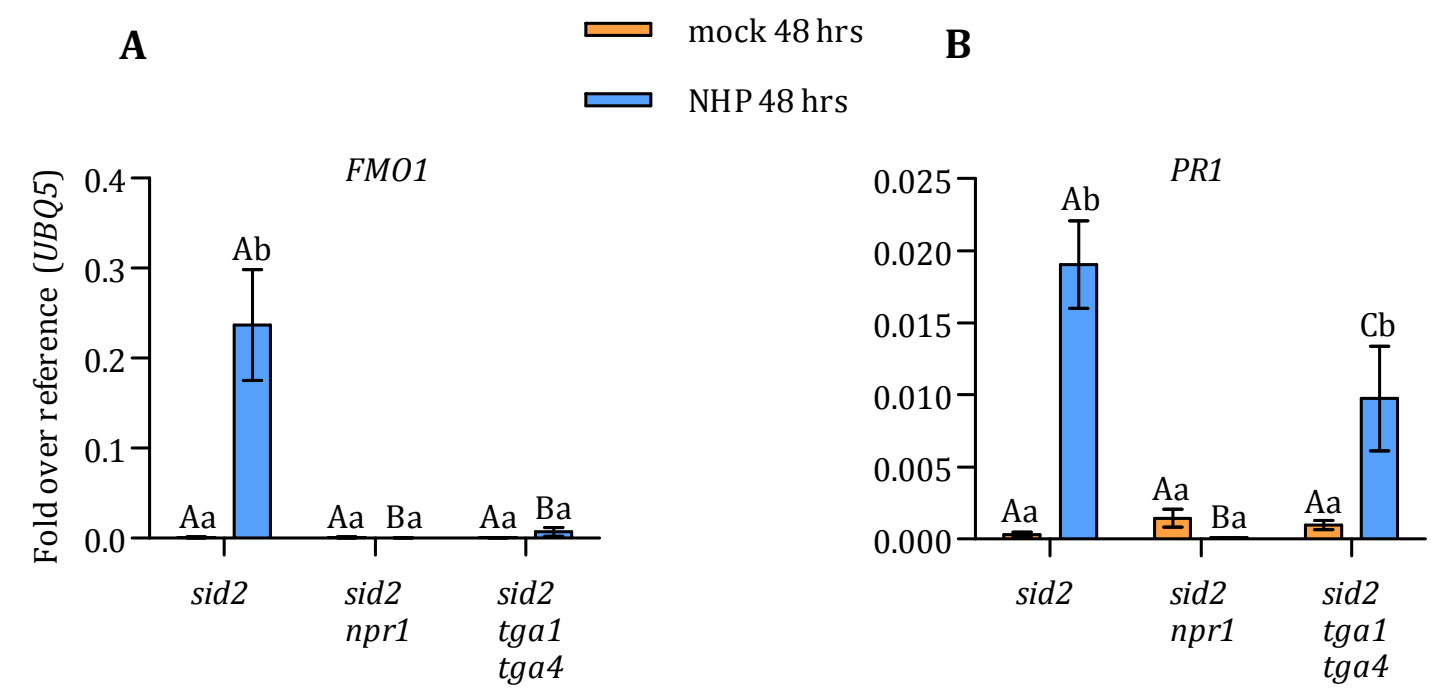

Figure 14: q-RT PCR analysis of FMO1 (A) and PR1 (B) in sid2, sid2 npr1, and sid2 tga1 tga4 plants following mock and NHP treatments. Three leaves of 4.5week-old plants were infiltrated with $\mathrm{MgCl}_{2}$ (mock) or $1 \mathrm{mM} \mathrm{NHP}$, and the leaves were collected after 48 hours. Transcript levels were normalized to the transcript levels of $U B Q 5$. Error bars represent the average \pm SEM of three plants of each genotype. Lowercase letters indicate significant differences $(P<0.05)$ between treatments performed with the same genotype; uppercase letters indicate significant differences $(P$ $<0.05$ ) between genotypes subjected to the same treatment. Statistical analysis was done using two-way ANOVA followed by Bonferroni's post-test.

Contrary to what was observed in Figure $12 \mathrm{~A}$, the sid2 mutant treated with NHP showed a prominent induction of FMO1 and PR1. The induction of FMO1 following NHP treatment was absent in the sid2 npr1 and sid2 tga1 tga4 mutants. A sixtyfold induction of $P R 1$ in the sid 2 mutant and a tenfold induction in sid2 tga1 tga4 was observed while the induction was absent in sid2 npr1. 
A

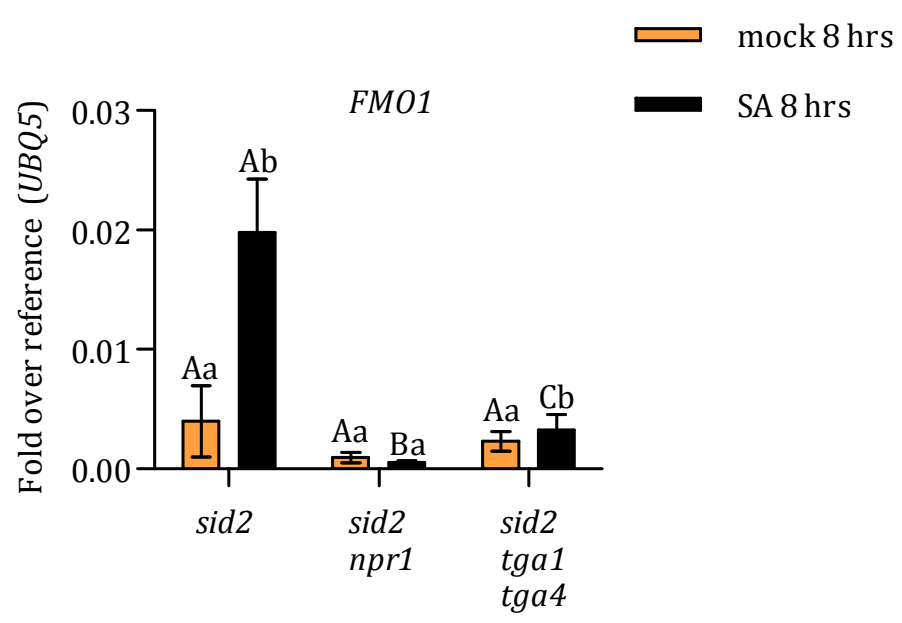

B

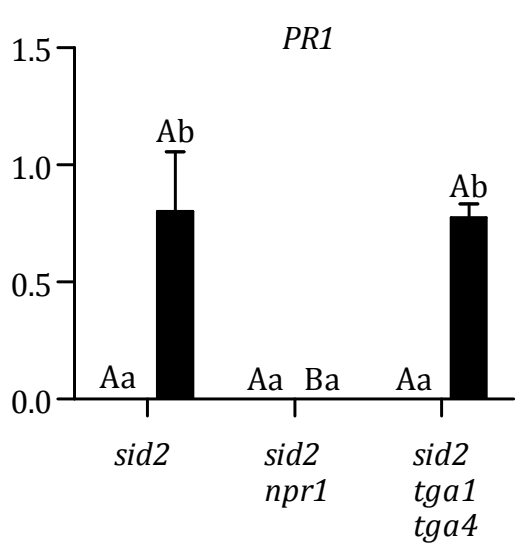

Figure 15: q-RT PCR analysis of FMO1 (A) and PR1 (B) in sid2, sid2 npr1, sid2 tga1 tga4 plants following mock and SA treatments. Three leaves of 4.5-weekold plants were infiltrated with $\mathrm{MgCl}_{2}$ (mock) or $1 \mathrm{mM} \mathrm{SA}$, and the leaves were collected after 8 hours. Transcript levels were normalized to the transcript levels of UBQ5. Error bars represent the average \pm SEM of three plants of each genotype. Lowercase letters indicate significant differences $(P<0.05)$ between treatments performed with the same genotype; uppercase letters indicate significant differences $(P<0.05)$ between genotypes subjected to the same treatment. Statistical analysis was done using two-way ANOVA followed by Bonferroni's post-test.

Based on the results from our pharmacological experiments, it is evident that NPR1 and TGA1/4 are required for the induction of FMO1 downstream of NHP, whereas the induction of $P R 1$ requires NPR1 but not TGA1/4.

As previously observed in Figure $13 \mathrm{~B}$, SA treatment led to the induction of FMO1 in the sid2 mutant. However, the induction was absent in the sid2 npr1 and sid2 tga1 tga4 mutants. Following 8 hours of SA treatment, PR1 induction was observed in sid2 and sid2 tga1 tga4 mutants, while the induction was absent in the sid2 npr1 mutant. Our data so far suggest that similar to NHP, SA functions via NPR1 and TGA1/4 leading to the induction of FMO1, whereas PR1 induction by SA requires NPR1 but not TGA1/4. 


\section{7) NHP treatment leads to NPR1 accumulation}

Given that NHP treatment results in the induction of genes in the sid2 mutant but not in the sid2 npr1 mutant, we questioned if NHP affects the protein accumulation of NPR1. To address this, the sid2 mutant was infiltrated with $\mathrm{MgCl}_{2}$

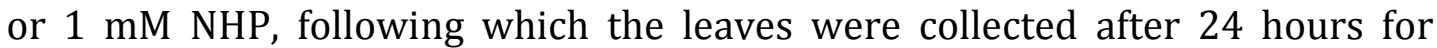
analysis.

The sid2 mutant infiltrated with NHP showed higher accumulation of NPR1 proteins as compared to the plants that received $\mathrm{MgCl}_{2}$ treatment. Subsequently, the FMO1 levels were checked in the sample to ensure that NHP treatment resulted in gene induction at this time point, we observed that the sid2 mutant showed a threefold induction of FMO1 upon NHP treatment, while sid2 npr1 mutant did not show an induction.

A

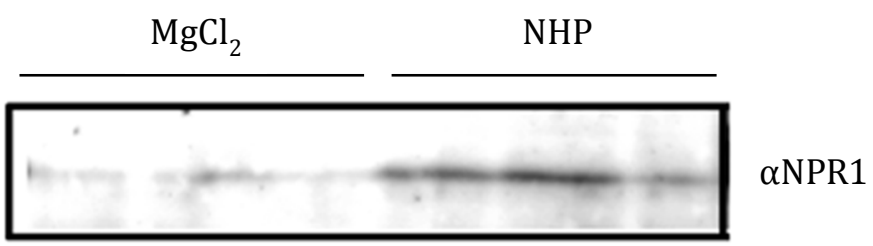

B

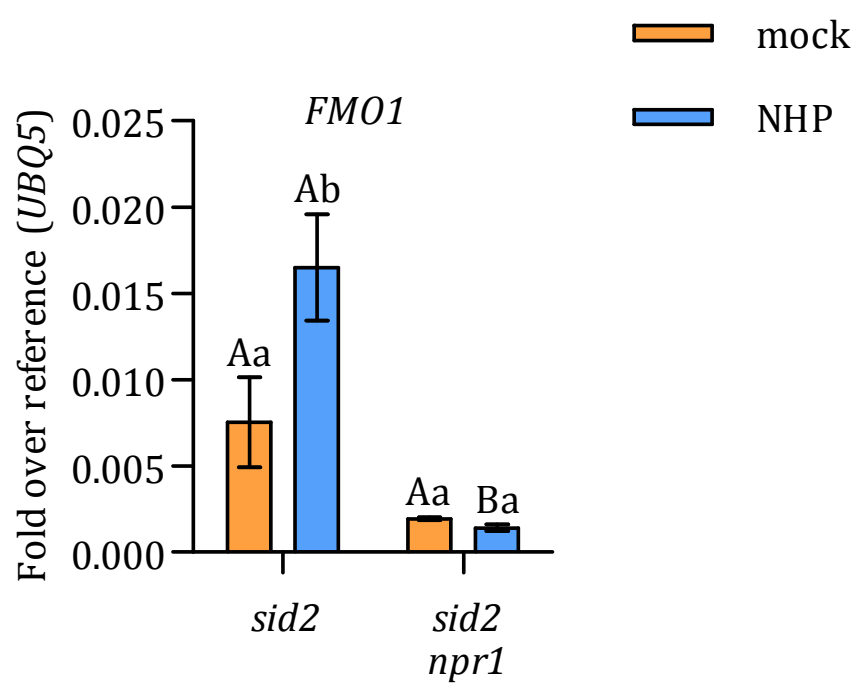


Figure 16: NPR1 protein levels in the leaves of sid2 mutant plants following $\mathrm{MgCl}_{2}$ and NHP treatments. (A) NPR1 protein levels in leaves of sid2 mutant plants following $\mathrm{MgCl}_{2}$ (mock) and NHP treatments. Three leaves were infiltrated with $\mathrm{MgCl}_{2}$ or $1 \mathrm{mM}$ NHP. The samples were collected after 24 hours for analysis. NPR1 protein levels were determined by Western blot analysis; three biological replicates were loaded. (B) Three leaves of 4.5-week-old plants were infiltrated with $\mathrm{MgCl}_{2}$ or $1 \mathrm{mM}$ NHP. Leaves were collected after 24 hours. Transcript levels were normalized to the transcript levels of $U B Q 5$. Error bars represent the average \pm SEM of three plants of each genotype. Lowercase letters indicate significant differences $(P<0.05)$ between treatments performed with the same genotype; uppercase letters indicate significant differences $(P$ $<0.05$ ) between genotypes subjected to the same treatment. Statistical analysis was done using two-way ANOVA followed by Bonferroni's post-test.

\section{8) EDS1 is required for the regulation of FMO1 by NHP}

The nucleo-cytoplasmic protein EDS1 has previously been reported to be essential for the induction of FMO1 and the accumulation of NHP following pathogen infection (Hartmann et al 2018) (Bartsch et al., 2006). We were interested in the possibility of EDS1 acting downstream of NHP accumulation. To address this, the eds1 mutant was included in the experiments with NHP infiltration.

On comparing the levels of FMO1 after $\mathrm{MgCl}_{2}$ and NHP treatment, we observed that the induction seen in Col-0 was absent in the eds1 mutant. A similar trend was observed in terms of the induction of PR1 and ICS1 as well, suggesting that the induction of genes following NHP treatment is dependent on EDS1. 
$\rightleftarrows$ mock $48 \mathrm{hrs}$

NHP $48 \mathrm{hrs}$

B

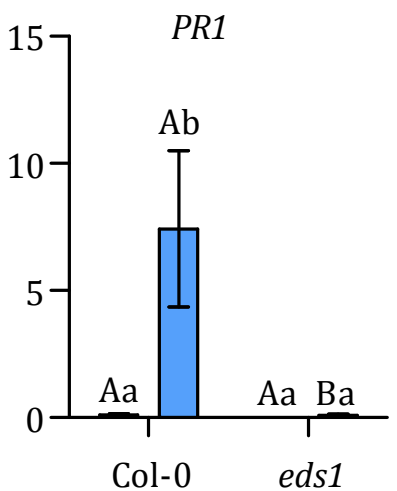

Figure 17: q-RT PCR analysis of FMO1 (A), PR1 (B), and ICS1 (C) in Col-0 and eds1 plants following mock and NHP treatment. Three leaves of 4.5-week-old plants were infiltrated with $\mathrm{MgCl}_{2}$ (mock) or $1 \mathrm{mM} \mathrm{SA}$, and the leaves were collected after 48 hours. Transcript levels were normalized to the transcript levels of UBQ5. Error bars represent the average \pm SEM of three plants of each genotype. Lowercase letters indicate significant differences $(P<0.05)$ between treatments performed with the same genotype; uppercase letters indicate significant differences $(P<0.05)$ between genotypes subjected to the same treatment. Statistical analysis was done using two-way ANOVA followed by Bonferroni's post-test.

The induction of FMO1 following SA treatment was slightly lower in the eds1 mutant as compared to Col-0. Whereas PR1 and ICS1 induction was attained to similar levels in Col-0 and eds1 mutant. 


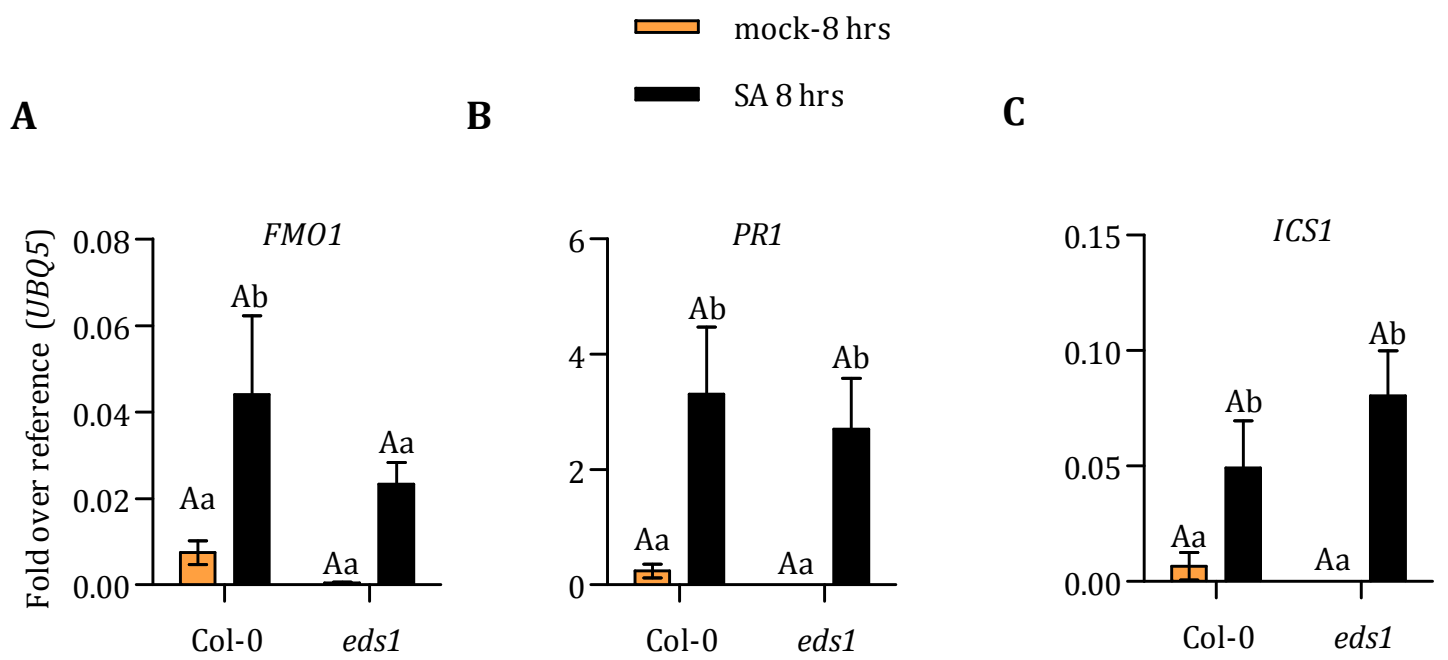

Figure 18: q-RT PCR analysis of FMO1 (A), PR1 (B), and ICS1 (C) in Col-0 and eds1 plants following mock and SA treatments. Three leaves of 4.5-week-old plants were infiltrated with $\mathrm{MgCl}_{2}$ (mock) or $1 \mathrm{mM} \mathrm{SA}$, and the leaves were collected after 8 hours. Transcript levels were normalized to the transcript levels of UBQ5. Error bars represent the average \pm SEM of three plants of each genotype. Lowercase letters indicate significant differences $(P<0.05)$ between treatments performed with the same genotype; uppercase letters indicate significant differences $(P<0.05)$ between genotypes subjected to the same treatment. Statistical analysis was done using two-way ANOVA followed by Bonferroni's post-test. 


\section{9) Primary infection with Psm potentiates the effect of NHP treatment}

Given the tendency of NHP to induce genes at late time points, we questioned if priming the plants by Psm infection could accelerate the ability of NHP to induce its target genes. To this end, lower leaves of sid2 and sid2 npr1 plants were infiltrated with or Psm (O.D. 0.005) or left untreated, two days after which the upper leaves were infiltrated with $1 \mathrm{mM} \mathrm{NHP}$ and samples were collected after 8 hours.

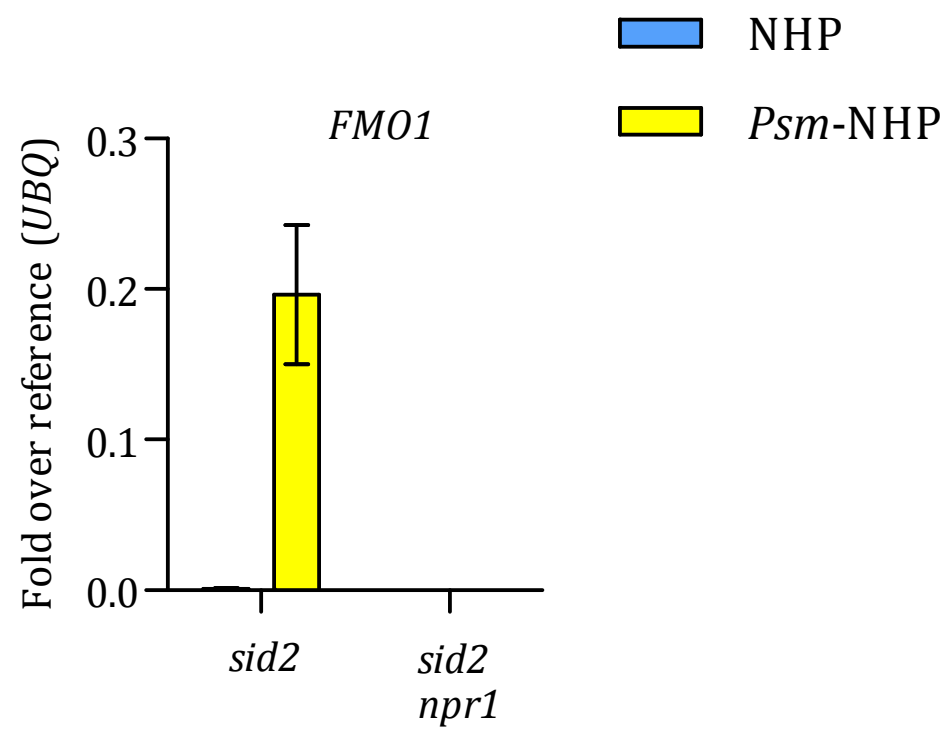

Figure 19: q-RT PCR analysis of FMO1 in sid2 and sid2 npr1 plants following NHP and Psm-NHP treatments. Three leaves of 4.5-week-old plants were either left untreated or infiltrated with Psm (O.D. 0.005), two days after which the systemic leaves were infiltrated with $1 \mathrm{mM}$ NHP. Leaves were collected after 8 hours. Transcript levels were normalized to the transcript levels of $U B Q 5$. Error bars represents the average \pm SEM of three plants of each genotype Lowercase letters indicate significant differences $(P<0.05)$ between treatments performed with the same genotype; uppercase letters indicate significant differences $(P<0.05)$ between genotypes subjected to the same treatment. Statistical analysis was done using two-way ANOVA followed by Bonferroni's post-test.

As observed in the previous experiments, NHP treatment did not result in gene induction at 8 hours time point. Plants that were initially primed with Psm showed induction of FMO1 after 8 hours of NHP treatment to levels similar to 
what is observed 48 hours after NHP treatment. As expected, the sid2 npr1 mutants did not show induction.

\subsection{0) Using Isothermal Titration Calorimetry (ITC) to study the NPR1 - SA interaction}

Various studies have reported that NPR1 acts as a receptor for SA (Ding et al., 2018; Kuai et al., 2015; Manohar et al., 2014; Wu et al., 2012). Most reports relied on size exclusion chromatography or equilibrium dialysis with radiolabelled SA followed by scintillation counting. We were interested in studying the role of NPR1 in NHP-mediated signaling pathway, and the question we were most interested in was if NPR1 is a receptor for NHP. Therefore, we aimed to set up a method which is easy to use and does not depend on radioactively labelled chemicals.

NPR1 protein with an N-terminal strep-MBP tag was expressed in insect cells using the Baculovirus Expression Vector System (BEVS) (Kost et al., 2005) (Methods section -5.1.11). Upon using $600 \mathrm{ml}$ of the insect cell culture for bacmid transfection, followed by an ÄKTA based StrepTRAPTM purification (Method section -5.4.6), we were able to enrich roughly $6 \mathrm{mg}$ of strep-MBP-NPR1 protein.

Using a partial digestion assay with trypsin, it has been reported that the binding of $\mathrm{Ca}^{2+}$ to the endoplasmic reticulum protein - calreticulin (CRT), leads to conformational change in the protein and this change could be visualized in terms of a difference in the digestion pattern caused due to trypsin treatment (Corbett et al., 2000). Initially, to study the interaction between strep-MBP-NPR1 and SA, we followed a similar protocol involving partial trypsin digestion. We hypothesized that - if the NPR1 protein efficiently binds SA resulting in a conformational change - the digestion pattern following binding might be different from the digestion pattern of the free protein. The NPR1 protein was incubated with SA and was treated with trypsin for a period of 5, 10 and $15 \mathrm{~min}$. As a control, NPR1 protein without SA was used. However, we did not observe a difference in the digestion pattern between NPR1 with and without SA. 
A

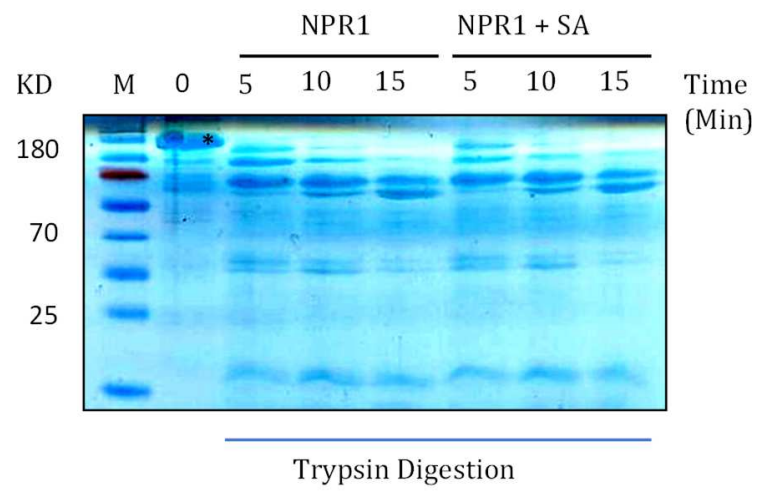

B
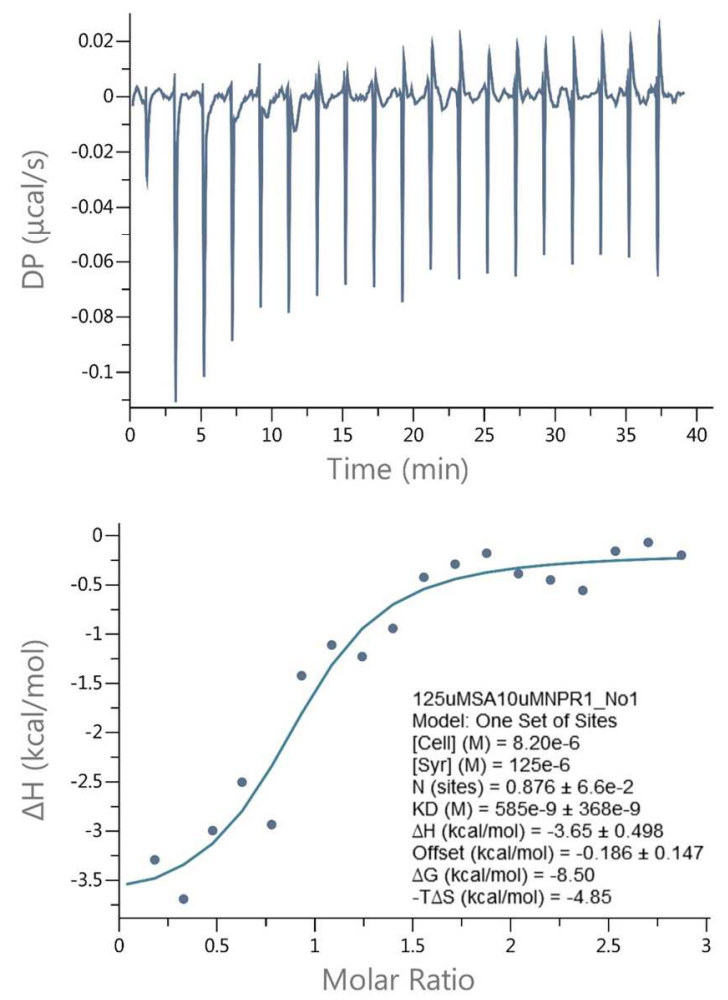

Figure 20: Analysis of NPR1-SA interaction using A) Partial digestion with Trypsin $2 \mathrm{mg} / \mathrm{ml}$ strep-MBPNPR1 protein in reaction buffer containing $50 \mathrm{mM}$ Tris, $\mathrm{pH}$ 8.0, $200 \mathrm{mM} \mathrm{NaCl}, 1 \mathrm{mM}$ TCEP was incubated with or without $1 \mathrm{mM}$ SA for $1 \mathrm{~h}$ on ice. Trypsin was added at a final concentration of $0.005 \mathrm{mg} / \mathrm{ml}$. The digestion reaction was carried out at the indicated time points at $20{ }^{\circ} \mathrm{C}$. Samples were loaded on a $10 \%$ SDS PAGE and stained with Coomassie blue.

B) Isothermal Titration Calorimetry (ITC) experiment performed for the quantification of interaction between NPR1 and SA. $125 \mathrm{uM}$ SA was titrated at 2 ul per injection for a total of 19 injections into a sample cell containing $400 \mu \mathrm{l}$ of $10 \mathrm{uM}$ strep-MBP-NPR1 at $25^{\circ} \mathrm{C}$. The strep-MBP-NPR1 protein and SA were in PBS buffer pH 7.4.

Following the failure of the trypsin digestion method, we decided to perform Isothermal Titration Calorimetry with NPR1 and SA. In the ITC experiment, 400 ul of $10 \mathrm{uM}$ strep-MBP-NPR1 protein was used in the sample cell, and $2 \mathrm{ul}$ of 125 uM SA was injected at an interval of 2 mins for a total of 19 injections. As the preliminary results with the ITC showed a Kd value of $585 \pm 368 \mathrm{nM}$ for the interaction between NPR1 and SA, it proves to be a suitable set up to study the interaction between NPR1 and NHP. 


\section{7) Results II - ROXY19 is involved in the repression of ORA59}

\section{1) The induction of ROXY19 expression during local and systemic immunity is partially SA-dependent}

Previous work from our lab has shown that ROXY19 expression is induced upon SA treatment and that ROXY19 interacts with class II TGA transcription factors TGA2, TGA5 and TGA6 (Ndamukong et al., 2007). Moreover, in the transcriptome analysis of SAR leaves performed by Bernsdorff et al., ROXY19 was amongst the genes that were strictly regulated by SA. We speculated that ROXY19 might be involved SAR-related defense responses (Bernsdorff et al., 2016).

First, we addressed the induction of ROXY19 after Psm infection in Col-0 and sid2 mutant plants following local Psm infection and Psm-infected SAR leaves. The response to local Psm infection was assayed by infiltrating the leaves with Psm at an OD of 0.005 and collecting the samples after a 24-hour time point (Figure 21). Infiltration with $10 \mathrm{mM} \mathrm{MgCl}_{2}$ (mock) was performed for control treatments. Infection with Psm resulted in an induction of ROXY19, which was partially dependent on salicylic acid, as revealed by the sid2 mutant, which showed partial impairment in the induction of ROXY19. The residual induction observed is probably due to the JA mimic coronatine that is produced by Psm. 

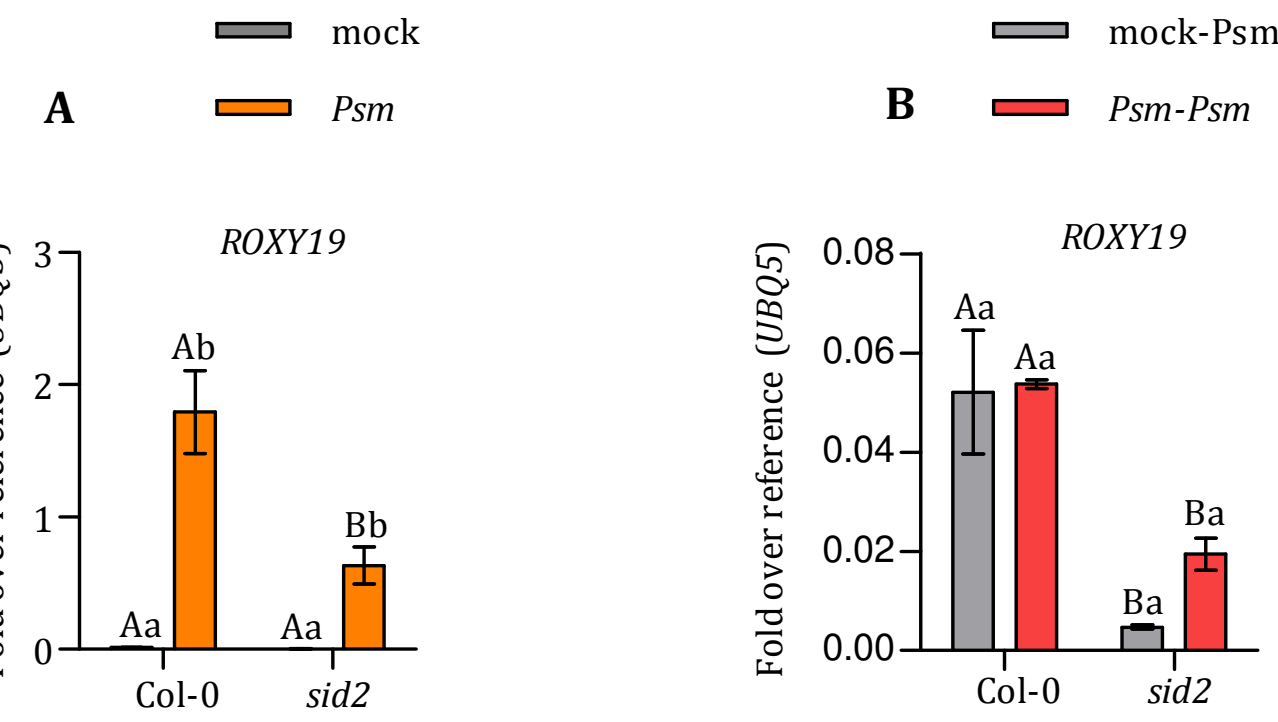

Figure 21: q-RT PCR analysis of ROXY19 in Col-0 and sid2 in (A) locally Psminfected leaves and (B) Psm-infected SAR leaves. For local infections, three leaves of 4.5-week-old plants were infiltrated with $10 \mathrm{mM} \mathrm{MgCl}_{2}$ (mock) or Psm (O.D. 0.005 ) and collected after 24 hours. For SAR experiments, the lower leaves of 4.5-weekold plants were infiltrated with $\mathrm{MgCl}_{2}$ (mock) or Psm (O.D. 0.005). Two days later, the systemic leaves were infiltrated with Psm (O.D. 0.005), and the systemic leaves were collected after 8 hours. Transcript levels were normalized to the transcript levels of $U B Q 5$. Error bars represent the average \pm SEM of five plants of each genotype. Lowercase letters indicate significant differences $(P<0.05)$ between treatments performed with the same genotype; uppercase letters indicate significant differences $(P<0.05)$ between genotypes subjected to the same treatment. Statistical analysis was done using two-way ANOVA followed by Bonferroni's post-test.

For the analysis of ROXY19 induction during SAR, the local leaves were infiltrated with $\mathrm{MgCl}_{2}$ or Psm (O.D. 0.005), two days after which the secondary leaves were infiltrated with Psm (O.D. 0.005). The secondary leaves were collected after 8 hours, leading to two different infections, hereafter referred to as mock-Psm and Psm-Psm. The levels of ROXY19 transcript in the mock-Psm treated sid2 samples were lower than the levels observed in the Col-0 plants (Figure 21); the same trend was observed in Psm-Psm treated plants. The lower relative transcript levels observed as compared to after local infection, are most likely due to the earlier time point analysed (8 hours versus 24 hours). 
7.2) ROXY19 is dispensable for the plant immune response against Psm

In the previous section of this thesis, we have shown that FMO1 is hyper-induced in the sid2 mutant and not induced in the tga 2 tga 5 tga6 mutant after Psm-Psm treatment (Figure $7 \mathrm{~A}$ ). Given that ROXY19 is less expressed in the sid2 mutant and that it can act as a repressor of class II TGAs, we questioned if the hyperinduction of FMO1 in the sid2 mutant might be due to lower ROXY19 levels

To address this question, we used the roxy18 roxy19 double mutant, which was generated in the lab using the CRISPR-Cas genome editing strategy. ROXY18 is the closest homologue of ROXY19 and shows a similar expression pattern in public data basis, the only difference being that its expression is rather repressed than induced by JA. However, FMO1 was induced to similar levels in Col-0 and the roxy18 roxy19 mutant. This suggests that ROXY18 and ROXY19 do not repress FMO1 transcription. Moreover, on checking the bacterial growth we observed a slightly higher susceptibility in the mock-Psm-treated roxy18 roxy19 mutants. Upon Psm pre-treatment, a clear SAR effect was seen in Col-0 and the roxy18 roxy19 mutant. It is concluded that although ROXY19 is induced in the course of Psm infections, it is not crucial for the regulation of immune responses against Psm. 


\section{mock-Psm}

A

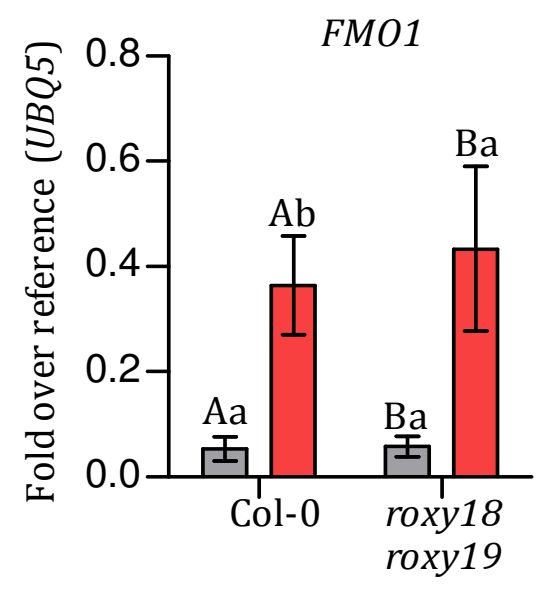

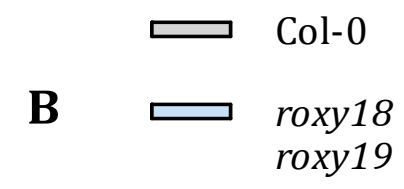

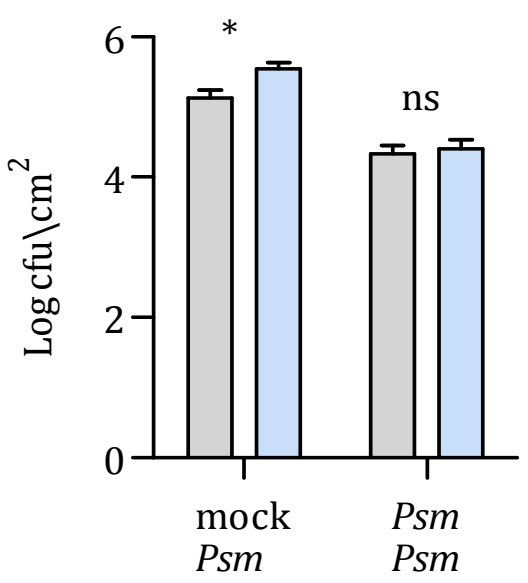

Figure 22: ROXY18 and ROXY19 is not involved in immune responses that are effective against Psm. (A) q-RT PCR analysis of FMO1 in Col-0 and roxy18 roxy19 in the systemic leaves during SAR. The lower leaves of 4.5-week-old plants were infiltrated with $\mathrm{MgCl}_{2}$ (mock) or Psm (O.D. 0.005). Two days later, the systemic leaves were infiltrated with Psm (O.D. 0.005), and the systemic leaves were collected after 8 hours. Transcript levels were normalized to the transcript levels of UBQ5. Error bars represent the average \pm SEM of five plants of each genotype. Lowercase letters indicate significant differences $(P<0.05)$ between treatments performed with the same genotype; uppercase letters indicate significant differences $(P<0.05)$ between genotypes subjected to the same treatment. Statistical analysis was done using two-way ANOVA followed by Bonferroni's post-test. (B) Bacterial growth in the systemic leaves of Col-0 and roxy18 roxy19 plants. The lower leaves of 4.5-week-old plants were infiltrated with $\mathrm{MgCl}_{2}$ (mock) or Psm (O.D. 0.005). Two days later, the systemic leaves were infiltrated with Psm (O.D. 0.001), and the systemic leaves were collected after 3 days post infection. Error bars represent mean values \pm SEM of colony forming units (cfu) per square centimeter from ten plants of each genotype. Each biological replicate consists of three leaf discs harvested from different leaves of one plant. Asterisks indicates statistically significant difference between genotypes $(P<0.05$; two-wayANOVA followed by Bonferroni's post-test). 


\section{3) ROXY19 is a negative regulator of ORA59 expression}

Previously, Zander et al. showed that the ectopic expression of ROXY19 leads to the repression of JA/ET-induced expression levels of the ORA59 gene, which encodes for a transcription factor essential for the majority of JA/ET-induced defense responses (Zander et al., 2012). Consistently, transcript levels of one of the target genes of ORA59, PDF1.2 are severely reduced. This correlates with a higher susceptibility of these plants, which might be due to the repression of the JA/ET pathway. To date, loss of function evidence that corroborates the role of ROXY19 in repressing ORA59 is not available.

As Psm infection leads to activation of the SA and JA signaling cascade (Zheng et al., 2012), it might provide a suitable system to study the role of ROXY19 in the SA-mediated antagonism of JA/coronatine-induced ORA59. To check the efficiency of the Psm-Arabidopsis pathosystem for studying the SA-JA cross-talk, we first analyzed the induction of ORA59 in sid2 mutants after Psm infection (Figure $23 \mathrm{~A}$ ).

Local Psm infection did not result in the induction of ORA59 in the Col-0 or sid2 plants, but the transcript levels were significantly higher (seven-fold) in the sid2 mutant. This indicates that already mock-induced SA levels lead to the repression of ORA59 transcription. However, under these conditions ROXY19 is not induced suggesting a ROXY-independent repression by SA in mock-treated plants. This was confirmed by the unaltered ORA59 transcript levels in the roxy18 roxy19 double mutant.

Upon Psm infection, a six-fold induction of ORA59 was observed in roxy18 roxy19 mutant, while the induction was absent in Col-0. The similarity of the ORA59 transcript patterns in Psm treated sid2 and roxy18 roxy19 mutants suggest that they act in one pathway with SA leading to elevated ROXY18/ROXY19 transcript levels and thus to repression of ORA59. 
A

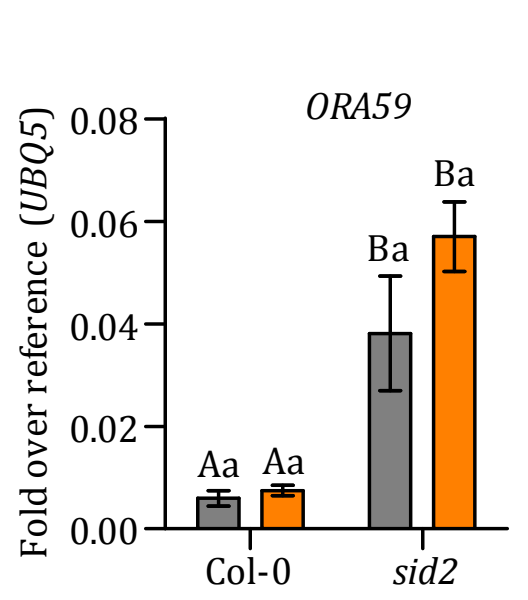

C
B

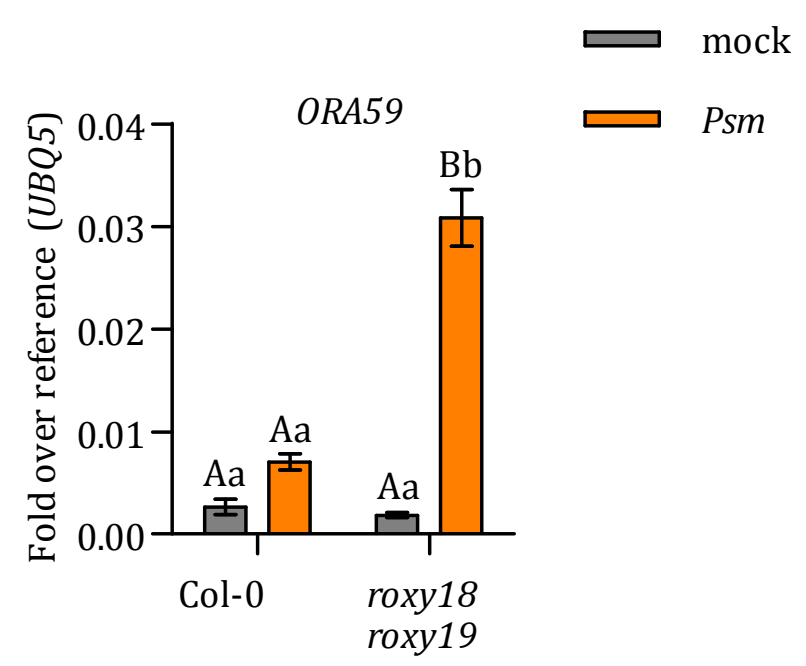

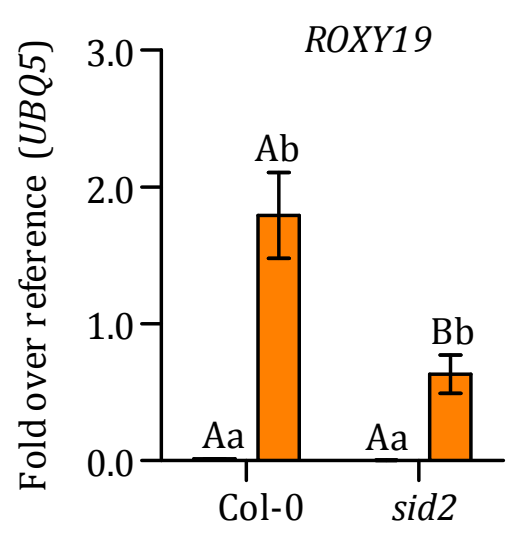

Figure 23: ROXY19 likely contributes to the supression of ORA59 trancription by SA q-RT PCR analysis of ORA59 transcript levels in Psm-infected Col0 and sid2 (A) and roxy18 roxy19 (B) and ROXY19 transcript levels in Psm-infected Col-0 and sid2 plants (C). Three leaves of 4.5-week-old plants were infiltrated with $\mathrm{MgCl}_{2}$ (mock) or Psm (O.D. 0.005) and collected after 24 hours. Transcript levels were normalized to the transcript levels of $U B Q 5$. Error bars represent the average \pm SEM of five plants of each genotype. Lowercase letters indicate significant differences $(P<0.05)$ between treatments performed with the same genotype; uppercase letters indicate significant differences $(P<0.05)$ between genotypes subjected to the same treatment. Statistical analysis was done using two-way ANOVA followed by Bonferroni's post-test.

Based on the above data, we concluded that ROXY18 and ROXY19 are involved in the SA- mediated repression of ORA59 during Psm infections. However, given that ORA59 levels were higher in the mock-treated sid2 plants as compared to Col-0 
but not in the roxy18 roxy19 plants, it suggests that SA exerts repressive effects in the mock-treated plants in a ROXY18/19- independent manner.

\section{4) The single cysteine (C186) in TGA2 is not required for the repression of ORA59}

In addition to evidence based on transgenic plants ectopically expressing ROXY19, our lab has shown in transient protoplast assays that ROXY18 and ROXY19 are capable of repressing ORA59 promoter activity. Moreover, the repressive effect of ROXY19 was not observed in the tga2 tga5 tga6 mutant protoplasts, suggesting that these TGA transcription factors are required for the repressive function of ROXY19 (Zander et al., 2012).

Similar to the roxy18 roxy19 and the sid2 mutants, the tga 2 tga 5 tga 6 mutant showed elevated ORA59 transcript levels in Psm-infected leaves (Figure18 A). This might suggest that ROXY18/19 and class-II TGAs form a repressive complex at the ORA59 promoter, as soon as ROXYs are induced by elevated SA levels. This repressive complex did not depend on the single cysteine at amino acid position 186 which is conserved in all three class II TGAs, as revealed by the ability of mutated TGA2 ${ }^{\mathrm{C} 186 \mathrm{~S}}$ to complement the phenotype.

However, ROXY19 transcript levels were lower in the tga 2 tga5 tga6 mutant as compared to Col- 0 and in the complementation line expressing TGA2 ${ }^{\mathrm{C} 186 \mathrm{~S}}$ (Figure 18 D). This, on the one hand, indicates that the cysteine is of functional importance when it comes to the activation capacity of TGA2 at the ROXY19 promoter. Assuming that the residual amounts of ROXY19 that are still expressed in the these lines contribute to the repression of ORA59, this result re-enforces the notion that the higher ORA59 transcript levels in the tga2 tga5 tga 6 mutant are due to impaired recruitment of ROXY18/19 to the ORA59 promoter. 
A

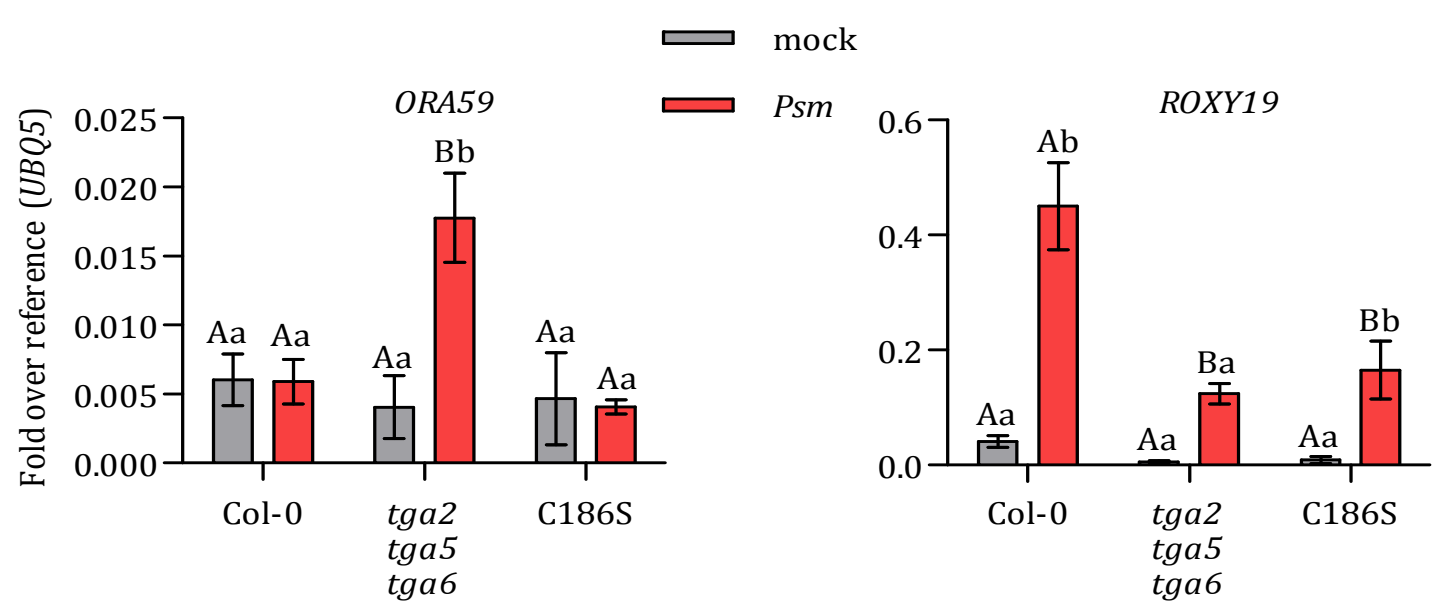

Figure 24: The conserved cysteine in TGA2 is dispensable for the repression of ORA59. q-RT PCR analysis of ORA59 (A) and ROXY19 (B) transcript levels in Col-0, tga256 and tga256 35S:TGA2 ${ }^{\text {C186S }}$ following local Psm infection. Three leaves of 4.5-weekold plants were infiltrated with $10 \mathrm{mM} \mathrm{MgCl}_{2}$ (mock) or Psm (O.D. 0.005) and collected after 10 hours. Transcript levels were normalized to the transcript levels of $U B Q 5$. Error bars represent the average \pm SEM of five plants of each genotype. Lowercase letters indicate significant differences $(P<0.05)$ between treatments performed with the same genotype; uppercase letters indicate significant differences $(P<0.05)$ between genotypes subjected to the same treatment. Statistical analysis was done using two-way ANOVA followed by Bonferroni's post-test.

Taken together, our preliminary results suggest that the conserved cysteine in TGA2 may play a role in the induction of ROXY19, but that it is dispensable for the repressive effect of ROXY19 on the ORA59 promoter. Though the exact mechanism of repression exerted by ROXY19 is still unknown, it is evident that class-II TGA transcription factors are essential for this repression. 


\section{5) SA-mediated repression of PDF1.2 during SAR does not depend} on ROXY18/19

Next, we analysed the induction of ORA59 in sid2 and roxy18 roxy19 after triggering SAR. The transcript levels of ORA59 were similar in the mock-Psm treated Col-0 and sid2 plants. Following Psm-Psm infection, transcript levels increased by a factor of two in the sid2 mutant, while Col-0 plants did not display an induction. These experiments support the notion that SA suppresses the JA/ET pathway. We next checked the transcript levels of PDF1.2, which is a target gene of ORA59. The level of PDF1.2 was constitutively high in the sid2 mutant as compared to Col-0 following mock-Psm and Psm-Psm treatment.

Having established that the absence of SA leads to higher induction of ORA59 in Psm-infected SAR leaves, we next analyzed if ROXY18 and ROXY19 are involved in the repression of ORA59 mediated by SA.

The transcript level of ORA59 was similar between Col-0 and roxy18 roxy 19 plants in mock-Psm-treated plants, which is similar to the situation when comparing Col-0 and sid2. In this experiment, the Psm-Psm treatment resulted in siginificantly higher ORA59 transcript levels already in Col-0 when comparing mock-Psm with Psm-Psm-treated plants. This increase was still higher in the roxy18 roxy19 mutant.

The transcript levels of PDF1.2 were fourfold higher in the mock-Psm-treated roxy18 roxy19 mutant as compared to Col-0. This was unexpected in view of our findings that ORA59 levels were not elevated. Following Psm-Psm treatment, transcript levels of PDF1.2 was repressed and were similar between Col-0 and roxy18 roxy19 plants, albeit ORA59 transcript levels were elevated.

Based on the above data, we concluded that ROXY18/19 are involved in the SAmediated repression of ORA59 during Psm-Psm infections. However, PDF1.2 seems to be regulated by ROXY18/19 during mock-Psm treatments, while SA 
exerts additional ROXY18/19-independent repressive effect on PDF1.2 in PsmPsm-infected plants.
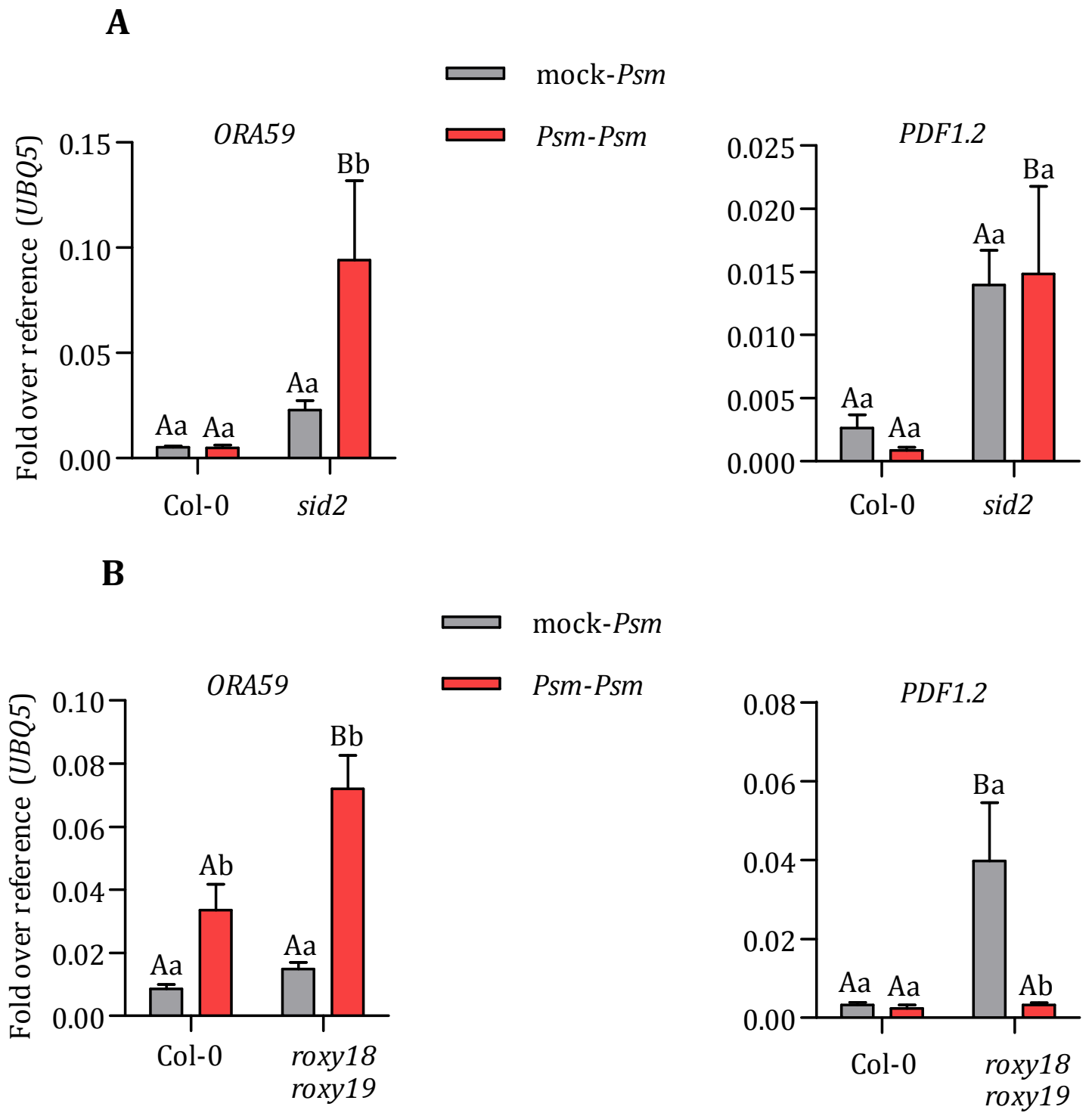

Figure 25: ROXY19 is not required for the suppression of PDF1.2 in Psminfected SAR leaves. q-RT PCR analysis of ORA59 and PDF1.2 transcript levels in Psminfected Col-0 and sid2 (A) and roxy18 roxy19 (B) plants. The lower leaves of 4.5-weekold plants were infiltrated with $\mathrm{MgCl}_{2}$ (mock) or Psm (O.D. 0.005). Two days later, the systemic leaves were infiltrated with Psm (O.D. 0.005), and the systemic leaves were collected after 8 hours. Transcript levels were normalized to the transcript levels of $U B Q 5$. Error bars represent the average \pm SEM of five plants of each genotype. Lowercase letters indicate significant differences $(P<0.05)$ between treatments performed with the same genotype; uppercase letters indicate significant differences $(P<0.05)$ between genotypes subjected to the same treatment. Statistical analysis was done using two-way ANOVA followed by Bonferroni's post-test. 


\section{6) ROXY19 is not involved in the repression of xenobiotic stress} response genes

Transcriptome analysis carried out using 35S:ROXY19 plants showed that genes related to the detoxification pathway are repressed in the transgenic lines. Over $40 \%$ of the repressed genes contain a TGACG binding site. Moreover, the 35S:ROXY19 and tga $\operatorname{tga} 5 \operatorname{tga} 6$ plants was found to be sensitive to xenobiotic stress induced by the electrophilic halogenated phenol 2,3,5-Triiodobenzoic acid (TIBA)(Huang et al., 2016). In our experimental set up, we did not see a hyperinduction of xenobiotic-stress related genes in the roxy18 roxy19 mutant either after mock treatment or Psm infections (Figure 26).

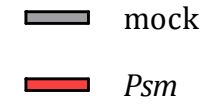

A

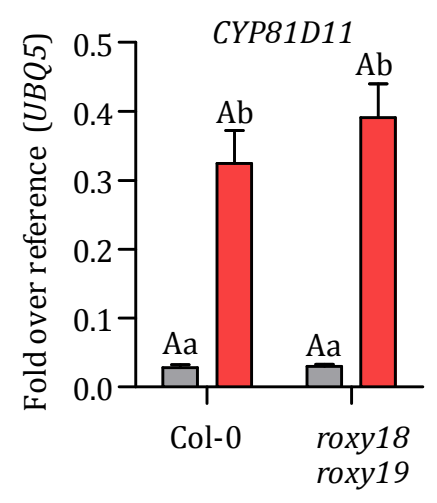

B

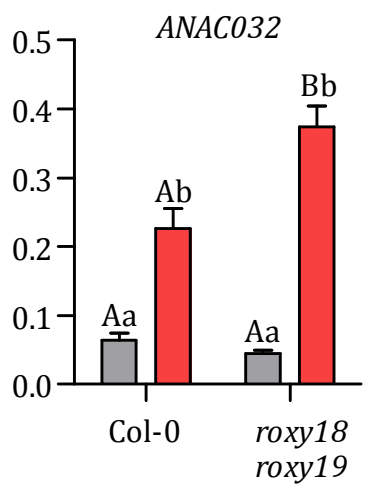

C

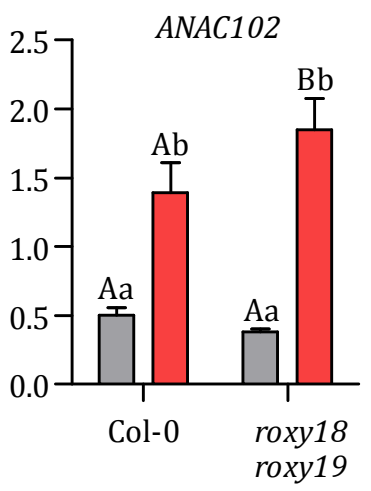

Figure 26: q-RT PCR analysis of CYP81D11 (A), ANACO32 (B) and ANACO12 (C) in Col-0 and roxy18 roxy19 plants. Three leaves of 4.5-week-old plants were infiltrated with $10 \mathrm{mM} \mathrm{MgCl}_{2}$ (mock) or Psm (O.D. 0.005) and collected after 24 hours. Transcript levels were normalized to the transcript levels of $U B Q 5$. Error bars represent the average \pm SEM of five plants of each genotype. Lowercase letters indicate significant differences $(\mathrm{P}<0.05)$ between treatments performed with the same genotype; uppercase letters indicate significant differences $(P<0.05)$ between genotypes subjected to the same treatment. Statistical analysis was done using two-way ANOVA followed by Bonferroni's post-test. 


\section{7) roxy18 roxy19 mutants are more resistant to B. cinerea infections}

Previous results from our lab have shown that plants ectopically expressing ROXY19 are more susceptible to the necrotrophic pathogen Botrytis cinerea. Moreover, the induction of ORA59, the gene product of which is a key regulator of the JA/ET signaling pathway, and its target gene PDF1.2 was reduced in the 35S:ROXY19 transgenic lines (Ndamukong et al., 2007; Zander et al., 2012). This led to the speculation that repression of the JA/ET pathway by ROXY19 leads to the higher susceptibility.

La Camera et al. had previously reported that the roxy18 T-DNA insertion mutant is more resistant to $B$. cinerea. However, this resistance was not related to elevated levels of PDF1.2 as a marker gene of the JA/ET defense pathway (La Camera et al., 2011). Here, we reproduced resistance phenotype of the roxy18 mutant with a CRISPR-Cas-derived plant line. Likewise, the roxy19 mutant was more resistant and the double mutant was even more resistant (Figure27). Similar to ourprevious experiments using Psm infection, the induction of ORA59 and PDF1.2 was higher in the roxy 18 roxy19 mutant after 48 hours of spraying the plants with $B$. cinerea. It seems likely that higher expression of the JA/ETinduced genes contribute to the elevated resistance. 
A

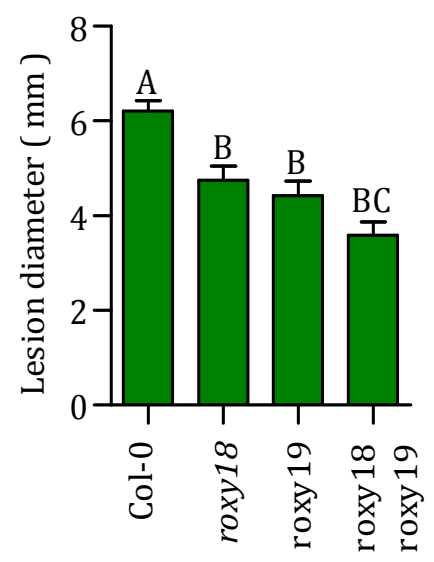

B

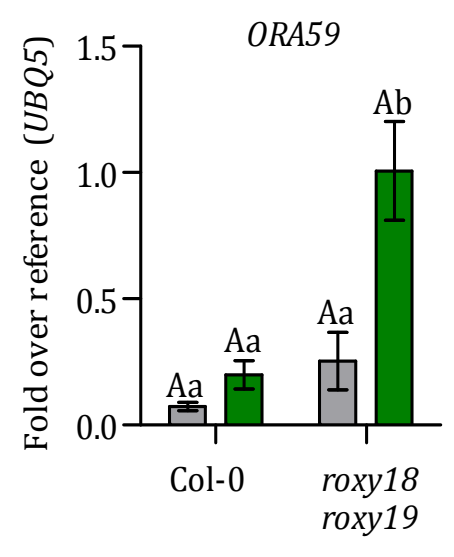

C

B.cinerea

Figure 27: Symptom development and gene expression in wild-type and roxy18 roxy19 mutant plants after $B$. cinerea infections. (A) Lesion sizes on wild-type and roxy18 roxy 19 mutant plants after 3 days of infection with B. cinerea. Sixweek-old plants were drop-inoculated with a $B$. cinerea spore solution $\left(5 \times 10^{4}\right.$ spores $\mathrm{ml}^{-1}$ ) or with quarter-strength potato dextrose broth. The diameters of at least 20 lesions per genotype were measured. q-RT PCR analysis of ORA59 (B) and PDF1.2 (C) in wildtype and roxy18 roxy19 mutant plants after 4 days of spray inoculation with B. cinerea. 4.5-week-old plants were sprayed with a B. cinerea spore solution $\left(2 \times 10^{5}\right.$ spores $\left.\mathrm{ml}^{-1}\right)$ or with quarter-strength potato dextrose broth (mock). Transcript levels were normalized to the transcript levels of UBQ5. Error bars represent the average \pm SEM of five plants of each genotype. Lowercase letters indicate significant differences $(P<0.05)$ between treatments performed with the same genotype; uppercase letters indicate significant differences $(P<0.05)$ between genotypes subjected to the same treatment. Statistical analysis was done using two-way ANOVA followed by Bonferroni's post-test 


\section{8) Discussion I - SA-independent processes in SAR}

Systemic acquired resistance (SAR) is an immune response program that isestablished in uninfected leaves after primary infection of a local leaf with biotrophic or hemibiotrophic pathogens (Conrath, 2006; Durrant and Dong, 2004). SAR leaves are more resistant to a wide range of pathogens. For decades, it was established that the phyotohormone salicylic acid (SA) was sufficient and necessary for SAR (Malamy et al., 1990; Molders et al., 1996). This thesis was based on published results showing that a mild SAR was still observed in plants lacking SA but not in plants lacking SA and the recently discovered regulatory metabolite NHP (Bernsdorff et al., 2016; Hartmann et al., 2018). The aim of the thesis was to start with the elucidation of SA-independent regulatory mechanisms during SAR. With FMO1, an important NHP biosynthesis gene, serving as a read-out for SA-independent induction of gene expression, the following main results were obtained: (1) FMO1 expression does not require SA, when plant are pre-treated with Psm, while it requires SA when plants are pretreated with $\mathrm{MgCl}_{2}$. (2) FMO1 expression requires the SA receptor NPR1 and the NPR1-interacting TGA transcription factors even in the absence of SA. (3) Induction of FMO1 by SA requires considerably less time than induction of FMO1 by NHP and induction of FMO1 by NHP is accelerated in SAR leaves. (4) Induction of FMO1 by NHP requires EDS1. (5) Recombinant NPR1 binds SA as shown by isothermal titration calorimetry.

\section{1) A mobile signal confers SA-independence on FMO1 expression in SAR leaves}

When comparing FMO1 expression in mock-Psm and Psm-Psm treated plants in Col-0 and sid2 plants (Figure 7), we observed that Psm-induced FMO1 transcript levels depended on SA in mock-pretreated plants but not in Psm-pretreated plants. This observation is different from previously published data (Bernsdorff 
et al., 2016), which document that Col-0 and sid2 are similar with respect to FMO1 expression in mock-Psm plants, while the twofold higher expression in sid2 compared to Col-0 in Psm-Psm-treated plants was the same as in our experiments. The reason for the discrepancy in the mock-Psm-treated samples is not clear, since a similar experimental setup was used except for the fact that Bernsdorff et al.,2016- collected their samples at 10 hours after the secondary infection, while we harvested at 8 hours. Since regulatory networks within the plant immune system are highly interconnected with other external factors, differences in e.g. light quality and quantity, the growth substrate or even the handling of plants by the person performing the experiment might lead to different reactions of the plant. Nevertheless, in our hands, the effect was highly reproducible and also observed in an independent $\mathrm{PhD}$ thesis (Budimir, 2019). It is concluded, that - in our hands - a mobile signal is generated in sid2 plants locally infected with Psm, which alters the signaling network in the systemic leaf in a way that FMO1 can now be induced after Psm infection in a manner that does not require SA (Figure 28 A/B). Whether this rewiring of the signaling cascade occurs at the level of NPR1, which is required for the SA-independent signaling cascade (see below), remains to be elucidated. 
A

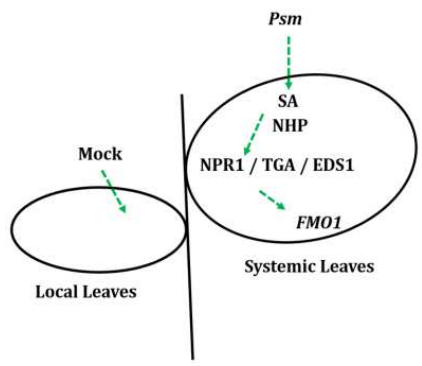

B

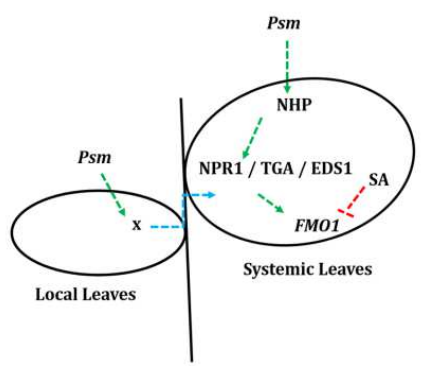

C

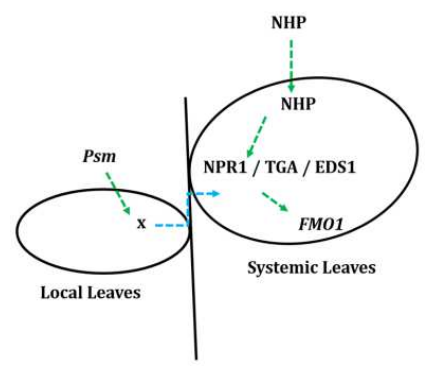

Figure 28: Graphical abstract - An unknown signal alters the signaling network leading to FMO1 induction in systemic leaves. Scenario A: Primary leaves infiltrated with $\mathrm{MgCl}_{2}$ and secondary leaves infiltrated with Psm. In this situation, the induction of FMO1 by Psm is dependent on NHP and the SA signaling cascade consisting of NPR1 and TGA transcription factors. Moreover, EDS1 is required for the induction of FMO1. Scenario B: Primary leaves infiltrated with Psm and secondary leaves infiltrated with Psm. The primary infiltration with Psm results in the generation of an unknown signal, which rewires the signaling cascade leading to FMO1 induction. The induction of FMO1 is now SA-independent but remains to be dependent on NHP, NPR1, TGA, and EDS1. Additionally, SA exerts an antagonistic effect on the induction of FMO1. Scenario C: Primary leaves infiltrated with Psm and secondary leaves infiltrated with NHP. The unknown signal generated upon primary Psm infection accelerates the NHPmediated induction of FMO1. The induction of FMO1 after NHP treatment depends on NPR1, TGA transcription factors and EDS1

\section{2) SA-independent FMO1 expression requires the SA receptor NPR1 and the NPR1-interacting TGA transcription factors}

Utilizing the sid2 npr1, sid2 tga 2 tga 5 tga 6 and sid 2 tga 1 tga 4 mutants, we provide unequivocal evidence for the requirement of NPR1 and interacting TGA factors for the SA-independent induction of FMO1 (Figure 10) (Figure $28 \mathrm{~B}$ ). This is noteworthy since it is well established that the NPR1/TGA regulatory module is operating in the SA signaling pathway (Johnson et al., 2003; Zhang et al., 2003). Since FMO1 expression is severely reduced in the sid2 ald1 mutant, it can be speculated that a pipecolic acid derivative, most likely NHP, is the regulatory metabolite that initiates an NPR1-dependent signaling cascade leading to FMO1 expression. Very recently, a similar observation has been made using the autoimmune mutant camta123, which lacks three redundant transcription factors 
that negatively regulate SA and NHP biosynthesis genes (Kim et al., 2019; Sun et al., 2019). FMO1 expression is constitutively induced in the camta123 mutant. Induction persists in the camta123 sid2 mutant but is severely reduced in the camta123 sid2 npr1 and the camta123 sid2 ald1 mutants. Moreover, it was reported that the accumulation of NPR1 protein was higher in the camta123 and camta123 sid2 mutants than in Col-0; this increase in accumulation was no longer present in camta123 sid2 ald1 and camta123 sid2 fmo1, suggesting that the NPR1 protein can accumulate in a system where SA is absent as long as the NHP biosynthesis pathway is active (Kim et al., 2019). In our experimental system, NPR1 protein levels in sid2 were more elevated after Psm-Psm treatment (which confers SA-independent FMO1 expression) than after mock-Psm treatment (which allows only SA-dependent FMO1 expression) (Figure 8). Moreover, NHP treatment led to increased NPR1 protein levels (Figure 16). Thus, NHP or possibly its mobile derivative NHP-glucose travelling from the Psm-infected local sid2 leaves might lead to increased NPR1 levels facilitating SA-independent induction upon Psm infections. Alternatively, increased NHP levels in the locally infected leaves (and the camta123 mutant) might initiate the synthesis of a yet unknown (mobile signal) which affects NPR1 protein levels. Based on the observation that NPR1 is not detected in the nuclear-enriched samples of the camta123 mutant, it was speculated that NPR1 might have a cytosolic role leading to the induction of NHP-dependent genes (Kim et al., 2019). However, based on our experiments, it is evident that TGA1/TGA4 and TGA2/TGA5/TGA6 are required for the SAindependent induction of FMO1 (Figure 10), suggesting that NPR1 acts as their co-activator in the nucleus. Moreover, using pharmacological treatments, we show that the sid2 tga1 tga4 mutant is impaired in the induction of genes following NHP treatment (Figure 14). If NPR1 has a cytosolic role as speculated, the contribution of TGA transcription factors would have to be explained in an NPR1-independent mechanism. We could rather imagine that FMO1 expression is only induced in a subset of cells of the camta123 mutant and that nuclear accumulation of NPR1 in these cells might not been seen in nuclear fractions prepared from the whole leaf. Expression of a GFP-tagged NPR1 derivative in the camta123 npr1 sid2 background might help to solve this question. Accordingly, 
introduction of such a construct into the sid2 npr1 mutant and subsequent SAR experiments might elucidate whether NPR1-GFP is enriched in the nucleus in uninfected and Psm-infected SAR leaves.

\section{3) Is NPR1 a receptor for NHP?}

So far, we have established that NHP and NPR1 are required for the induction of FMO1 in the absence of SA (Figure 10). The next obvious question is the mechanistic aspect of the interaction between NPR1 and NHP. As mentioned in the introduction, Wu et al. showed NPR1 binding to SA and proposed that $\mathrm{Cu}^{2+}$ coordinated by Cys521 and Cys529 facilitated binding of SA (Wu et al., 2012). More recently, Ding et al. provided genetic and biochemical evidence towards the requirement of a conserved amino acid residue Arg432 in NPR1, which, when mutated to glutamine (R432Q), disrupts the ability of the protein to bind SA, but does not hinder its interaction with transcription factor TGA2 (Ding et al., 2018). In this thesis, we provide evidence that the conserved arginine residue is also required for the regulation of FMO1 during SAR (Figure 9). This conclusion is based on the observation that the complementation line that expresses NPR1 $1^{\mathrm{R} 432 \mathrm{Q}}$ is in the $n p r 1$ background was still impaired with respect to FMO1 expression in Psm-Psm-treated plants. Although it would be advantageous to perform the complementation in the $n p r 1$ sid2 background, we can comment with a high degree of confidence that the arginine residue which is required for SA binding plays a critical role in the induction of FMO1. Based on the structural similarity between SA and NHP (Figure 3), this might indicate that NHP directly binds to NPR1. In order to challenge this hypothesis, we have established the ITC method to monitor NPR1-ligand interactions, but NHP binding experiments still have to be done.

The occurrence of a single receptor binding multiple ligands has not been reported in plants before. Studies on human immune signaling have shown that the Toll-like receptor TLR4 in macrophages acts as a receptor for bacterial 
lipopolysaccharides (LPS) as well as Tenascin-C, which is an extracellular protein present in the host. Binding of either ligand to TLR4 leads to a ligand-specific response, binding of LPS leads to the activation of the immune response, whereas Tenascin-C binding to TLR4 leads to the initiation of the repair process (Piccinini et al., 2016).

A similar scenario can be envisioned in terms of the receptor NPR1 and SA and NHP. SA binding to NPR1 results in the induction of its target genes such as PR1, which, based on our pharmacological experiments, cannot be induced by NHP treatment to the same extent in the absence of SA, suggesting that PR1 induction is an SA-specific response that requires NPR1 (Figure 12). On the other hand, NHP binding to NPR1 results in the induction of its target genes such as FMO1 and $A L D 1$. However, there seems to be an overlap here as $S A$ can also induce FMO1 and $A L D 1$ in an NPR1-dependent manner.

Accumulation of SA causes a redox shift in the cells, which leads to the conversion of NPR1 from the oligomeric state to the monomeric state facilitated by the reduction of intermolecular disulfide bonds by the thioredoxin TRXh5 (Mou et al., 2003; Tada et al., 2008a). The monomeric form of NPR1 translocates into the nucleus leading to the induction of SAR-related genes. Previously, Mou et al. reported that mutation of the cysteines C82 and C216 to alanine resulted in the detection of constitutive NPR1 monomers. It was further shown that in npr1 plants expressing NPR1 ${ }^{\mathrm{C} 82 \mathrm{~A}}$ or NPR1 ${ }^{\mathrm{C} 216 \mathrm{~A}}, P R 1$ is constitutively induced, thereby suggesting that the monomerization of NPR1 is sufficient for the induction of PR1. However, the authors also report that treating the mutants with SA resulted in an increase in the transcript levels of $P R 1$ and that the mutants were not as resistant to Psm as compared to SA-treated wild type plants (Mou et al., 2003). Taken together, their results suggest that the monomerization of NPR1 is a prerequisite for induction of gene expression and that SA is required for the realization of full expression levels.

At least two reports are suggesting that the localization of NPR1 to the nucleus can take place without the induction of SA production. Firstly, Lai et al. reported 
that initiation of the Unfolded Protein Response (UPR) results in a shift in the redox state of the cell, which leads to the nuclear localization of NPR1(Lai et al., 2018). Although the experiments have not been performed in the sid2 background, they show that an increase in SA accumulation is not observed following UPR. The second line of evidence is from the publication by Xiong et al., who showed that mutation in a plastidic 6-phospogluconolactonase results in a decrease in the redox potential of the cell. The pgl6 mutant shows constitutive expression of PR1 and enhanced defense response. On checking nuclear accumulation of NPR1 in the pgl6 mutant background, a higher accumulation was observed even in naïve plants. Interestingly an increase in free SA production was not found in this mutant, but levels of the SA-glucoside (SAG) were elevated (Xiong et al., 2009). It has to be mentioned that when pgl6 mutants were crossed with sid2, the constitutive PR1 induction and enhanced resistance phenotype was lost, which corroborates with the findings from Mou et al. - that nuclear localization of NPR1 is sufficient for PR1 induction, but SA or SAG further boost the induction of gene expression. As mentioned above, the generation of GFPtagged NPR1 lines in sid2 npr1 mutants would provide a suitable system to address if NPR1 can accumulate in the nucleus in the absence of SA.

In our pharmacological experiments, we have observed that the NHP treatment of sid2 plants results in the induction of TRXh5 (Supplement Figure 2). Given that TRXh5 catalyzes the conversion of NPR1 oligomers into monomers (Tada et al., 2008a), and that it can be induced in the absence of SA by NHP, it can be envisioned that following NHP accumulation the NPR1 protein is monomerized and translocated into the nucleus in an SA-independent manner.

The interaction between NHP and NPR1 is likely not the same as that reported for SA and NPR1, the reason for this assumption being that SA treatment leads to early induction of FMO1 in an NPR1-dependent manner, followed by a decrease in expression (Figure 13), while NHP treatment leads to induction only after 48 hours, concomitant with increased ICS1 expression. Conspicuously, the kinetics can be accelerated by infecting plants with Psm and treating the systemic leaves after 48 hours with NHP (Figure 19), (Figure 28 C). We have postulated above 
that local infection with Psm leads to NHP synthesis, which leads to the synthesis of a mobile signal that primes the NPR1-dependent signaling cascade to respond to NHP, which in turn is generated upon secondary infection with NHP. This process, which takes about 48 hours might also be initiated by NHP treatment: Application of NHP would lead to the generation of the signal which primes the NPR1-dependent signaling cascade to respond to the applied NHP. Metabolite analysis of petiole exudates capable of facilitating accelerated NHP responses might be a way to identify this unknown intermediate leading to efficient NHPinduced NPR1-dependent gene expression. However, even knowledge of the metabolite would leave open the question how priming is realized at the molecular level. Recently, Formaldehyde-Assisted Isolation of Regulatory Elements (FAIRE) (Baum et al., 2019) experiments have shown that the chromatin of the FMO1 promoter is more open in SAR leaves and that chromatin opening requires NPR1. If this mechanism would account for the acceleration of the NHP-responsiveness of FMO1 expression in SAR leaves, one would have to speculate that the unknown intermediate opens the chromatin in an NPR1dependent manner, so that the promoter can react rapidly to NHP through an NPR1-dependent or NPR1-independent manner. If NHP is infiltrated into naïve plants, the process of generation of a NHP-dependent metabolite and the subsequent opening of the chromatin would explain why induction takes so long. At the same time, one has to postulate that all these processes can also be triggered directly by SA, which induces FMO1 with a fast kinetics in naïve plants.

\section{4) EDS1 is required for activation of target genes by NHP}

This thesis has provided evidence that EDS1 is important for NHP-mediated induction of FMO1 (Figure 18). The EDS1 protein and its interaction partner PAD4 are essential for the actuation of basal and $\mathrm{R}$ gene-mediated resistance against biotrophic and hemibiotrophic pathogens and for the establishment of SAR (Wiermer et al., 2005; Wittek et al., 2014). A series of petiole exudate transfer experiments showed that EDS1 is required for the generation as well as the 
perception of the mobile signal during SAR (Breitenbach et al. 2014). When petiole exudates collected from Pst RPM1 infiltrated Col-0 leaves were infiltrated into a different set of Col-0 plants, induction of the PR1 transcription was observed. However, when the exudates from Col-0 plants were infiltrated into the eds1 mutant the induction was not attained. Similarly, when exudates from infected eds1 mutants were infiltrated into wt plants, $P R 1$ induction was absent. It would be very interesting to repeat these experiments and to monitor priming of NHP-induced FMO1 expression.

At this stage, we do not have further evidence to strengthen the notion of an EDS1 regulatory node in the signaling cascade mediated by NHP; however, this does not stop us from speculating. We envision the possible scenarios by which EDS1 can contribute to the signaling cascade downstream of NHP:

1) The EP domain of EDS1 consists exclusively of $\alpha$-helical sheets; the arrangement of this order is known to provide extended surface area and potential flexibility for interaction with a wide range of partners (Groves and Barford, 1999). This makes EDS1 a putative candidate that could bind NHP and start the synthesis of the mobile signal that primes NPR1 to respond to NHP. Alternatively, the cavity that is formed by the EDS1PAD4 heterodimer might accommodate NHP (Bhandari et al., 2019).

2) EDS1 is possibly required for priming NPR1 so that it can respond to NHP. In the absence of EDS1, NPR1 might not efficiently bind NHP, and the signaling might be disrupted. However, it is worth mentioning that SA treatment led to the induction of FMO1 and PR1 in the eds1 mutant, while npr1 was devoid of this induction. If EDS1 would be essential for maintaining the function of NPR1, it should be deficient in the SAmediated signaling cascade as well. Given that this is not the case, it would mean that EDS1 might have a role in regulating NPR1 specifically for NHP to function, while signaling downstream of SA does not require regulation of NPR1 by EDS1. 
8.5) ITC might be used to characterize the interaction between NPR1 and SA

Based on the preliminary experiments shown in Figure 20 B, ITC might be a way to characterize SA binding to NPR1 in more detail and to challenge the hypothesis whether NPR1 can directly bind to NHP. ITC as a not yet utilized method to study SA binding to NPR1 might be useful to add another independent piece of information to the controversy that exists in the literature concerning the question whether and how NPR1 binds SA. In 2012, Fu et al., reported that NPR3 and NPR4 but not NPR1 bind SA(Fu et al., 2012). The NPR proteins were expressed as fusion proteins with glutathione $S$-transferae in $E$. coli and purified using Glutathione Magnetic Beads. The protein-bound beads were incubated in with [3H]-SA, and radioactivity bound to the beads was measured. Having NPR3 and NPR4 as positive controls, the statement that NPR1 does not or only weakly bind SA seemed plausible. In the same year, using equilibrium dialysis, Wu et al described that NPR1 binding to SA could not be detected by methods using NPR1 bound to a solid phase, but that equilibrium dialysis showed SA binding with one molecule SA bound to one molecule NPR1 with a Kd of $137 \mathrm{nM}(\mathrm{Wu}$ et al., 2012). In 2018, using size exclusion chromatography to separate free SA from the NPR1SA complex, Ding et al. reported SA binding to NPR1 (Ding et al., 2018). However, in this assay, only $0.02 \%$ of NPR1 was able to bind SA. Ding et al. questioned the findings of Wu et al. on the importance of two cysteines for SA binding, which are not conserved in NPR sequences from other species. Using NPR1 purified from insect cells and using ITC as a binding assay, we are now able to clarify the question of whether $\mathrm{Cys}^{521}$ or $\mathrm{Cys}^{529}$ are important for SA binding. Moreover, we can challenge the hypothesis whether NPR1 binds NHP. 


\section{9) Discussion II - ROXY18 and ROXY19 are involved in SA-mediated repression of ORA59}

The glutaredoxin ROXY19 was isolated in yeast two hybrid screens designed to find interaction partners of TGA2 (Ndamukong et al., 2007). Expression of ROXY19 and its closest homologue ROXY18 is induced upon SA treatment, and the ectopic expression of ROXY19 results in the repression of JA/ET-induced expression of ORA59 and PDF1.2, implying that ROXY19 is involved in the antagonistic effect of SA on the JA/ET pathway (Ndamukong et al., 2007; Zander et al., 2012). So far, most of the evidence that is built around the role of ROXY18 and ROXY19 function comes from transient protoplast assays and transgenic plant lines ectopically expressing ROXY19. In this thesis, we provide loss of function evidence that supports the previously assumed roles of ROXY18 and ROXY19 as negative regulators of ORA59 expression.

\section{1) ROXY18 and ROXY19 are not involved in the regulation of FMO1}

Consistent with published data, we have shown that Psm-Psm-treated sid2 mutants show higher induction of FMO1 as compared to Col-0 (Figure 7). This shows that SA exerts a negative effect on the FMO1 promoter during the establishment of SAR. The reasons as to why ROXY18/ROXY19 were likely candidates for the SA-mediated repression were as follows:

1) ROXY18 and ROXY19 are induced upon SA treatment.

2) Over expression of ROXY19 led to the repression of TGA-regulated genes.

3) Transient expression analyses in protoplasts have shown that ROXY19 represses promoters regulated by TGA factors and FMO1 is regulated by TGA factors (Figure 10).

However, upon performing SAR experiments with the roxy18 roxy19 mutant, we did not observe a difference in expression between Col-0 and the roxy18 roxy19 
mutant. The bacterial growth assay suggests that roxy18 roxy19 supports slightly higher bacterial levels in the mock-Psm treatment. This effect was too minute for us to focus on (Figure 22).

\section{2) ROXY19 and ROXY18 are involved in repression of ORA59 promoter activity}

Due to the small size of the ROXY genes, T-DNA insertion mutants are rare and the expansion of the gene family make functional redundancy likely. Up to now, the following loss-of-function phenotypes were available: control of floral organogenesis (ROXY1 (Li et al., 2009)), microspore formation (ROXY1 and ROXY2 (Murmu et al., 2010)), systemic regulation of nitrate uptake (ROXY6 and ROXY9 (Ohkubo et al., 2017)), root architecture in response to nitrate (ROXY1115 (Walters and Escobar, 2016)), defense against the biotrophic pathogen Botrytis cinerea (ROXY18 (La Camera et al., 2011)), and tolerance against photooxidative stress (ROXY18 (Laporte et al., 2011)). However, it has to be emphasized that - except for the floral phenotypes of the roxy1 and roxy1 roxy2 mutants - phenotypes seem to be rather subtle. Here, we added another phenotype, which is repression of the JA/ET pathway through repression of ORA59 (ROXY18/ROXY19).

Thanks to the CRISPR-Cas genome editing technology, a roxy18 roxy19 double mutant became available in our lab. In this mutant, we were able to detect significantly elevated ORA59 transcript levels under the following treatments: infiltration with Psm (Figure 23) infiltration of SAR leaves with Psm (Psm-Psm) (Figure 25) and upon spray inoculation with $B$. cinerea (Figure 27). It can be thus concluded that ROXY18 and ROXY19 have a negative effect on ORA59 promoter activity.

However, further experiments are needed to show whether ROXY18/ROXY19 are indeed involved in the SA/JA-ET antagonism. Support for this hypothesis comes 
from Psm-treated plants: ORA59 transcript levels are higher in sid2 (7-fold) and the roxy18 roxy19 double mutant (6-fold) as compared to Col-0 and this correlates with reduced ROXY19 transcript levels in sid2. Since in contrast to ROXY19 transcript levels, ROXY18 transcript levels are not induced by Psmderived coronatine, it can be expected that they are more strongly reduced in sid2 than ROXY19 transcript levels. Pharmacological treatments of plants with JA/ET and SA might yield a clearer picture. In this experimental set-up, SA lowers JA/ETinduced ORA59 transcript levels. However, previous studies in the lab using a roxy18 roxy19 roxy20 triple mutant that had been obtained by crossing single mutants from different ecotypes, had not supported the notion that these ROXYs are mediates of the SA-JA/ET antagonism. It might well be that ROXYindependent mechanisms are responsible for the cross-talk seen in pharmacological experiments, while ROXYs are important for the cross-talk upon Psm infections. Reproduction of the preliminary results presented in this thesis by independent experiments of Psm-infected sid2, roxy18, roxy19, and roxy18 roxy19 mutants might yield a more robust data set on the role of these ROXYs in the regulation of ORA59 expression.

Similar to the sid2 and the roxy18 roxy19 mutant, the tga 2 tga 5 tga 6 mutant showed increased ORA59 expression after infection of local leaves with Psm (24 hours) (Figure 24). This phenotype might either be explained by the absence of TGA factors as sites of ROXY recruitment to the ORA59 promoter or by the lower expression of ROXYs. Indeed, at least ROXY19 expression is as reduced in tga2 tga 5 tga 6 as in sid2. Unexpectedly, expression of TGA2 ${ }^{\mathrm{C} 186 \mathrm{~S}}$ in the tga2 tga 5 tga 6 mutant rescued repression of ORA59, but not induction of ROXY19. Since we envision that the exchange of a cysteine to a serine mimics the permanently reduced form of TGA2, this result suggests that the reduced form of TGA2 can repress the ORA59 promoter even in the absence of ROXYs, while it is unable to activate the ROXY19 promoter. In other words, TGA2 in its oxidized (glutathionylated?) version activates the ROXY promoters, leading to ROXY expression which in turn leads to the reduction of C186 of TGA2 at the ORA59 promoter finally mediating repression. However, this regulation does not affect 
other TGA2-regulated promoters like e.g. FMO1 or CYP81D11. Therefore, biochemical analysis of the redox state of TGA2 is hampered by the fact that changes might only happen at the ORA59 promoter. Moreover, it has to be mentioned that the experiments are preliminary and need to be reproduced including the 35S:TGA2 line as a control for the 35S:TGA2 ${ }^{286 S}$ line. Ideally, the mutations should be introduced into genomic TGA2 constructs.

Another aspect that is worth discussing is the expression pattern of PDF1.2 in mock-Psm- and Psm-Psm-treated plants (Figure 25). Under both conditions, PDF1.2 expression was higher in the sid2 plants, corroborating the published negative effect of SA on PDF1.2 expression. Interestingly, enhanced levels of PDF1.2 were only observed in the mock-Psm-treated roxy18 roxy19 double mutant but not in the Psm-Psm-treated double mutant. The mechanisms underlying this ROXY18/ROXY19-independent repression mechanism that occurs although ORA59 transcript levels are hyper-activated after Psm-Psm treatment, remains to be elucidated. A previous publication suggested SAmediated degradation of the ORA59 protein (Van der Does et al., 2013).

Finally, enhanced ORA59 expression was observed upon infestation of roxy18 roxy19 plants with the necrotrophic fungus Botrytis cinerea (Figure 27), which induces both SA- and JA/ET-dependent defense responses at the early stages of infection. Whether the observed increased resistance of the roxy18 roxy19 mutant is indeed due to the hyperactivation of the JA/ET pathway can be analyzed after generation of the ora59 roxy18 roxy19 triple mutant. Since ROXYs are potential regulators of the redox homeostasis they might alter the levels of reactive oxygen species, which in turn would trigger JA/ET-independent effects leading to susceptibility.

The mechanism of action of ROXYs has remained enigmatic. On the one hand, at least 15 of the 21 ROXYs, including the highly expressed ROXY4 and ROXY10, can repress the ORA59 promoter in transiently transformed protoplasts (Zander et al., 2012). To explain the fact that loss of ROXY18 and ROXY19 leads to a phenotype concerning ORA59 expression it has to be postulated that they are 
Discussion

expressed in different cell types. Another question is why the CC motif in the active center is conserved in all 21 ROXYs. 


\section{0) Summary I - SA-independent processes in systemic acquired resistance (SAR)}

SAR is an immune response that is established in the systemic leaves after infection of local leaves with biotrophic or hemibiotrophic pathogens. According to the current model, the amino acid-derived metabolite $\mathrm{N}$-hydroxypipecolic acid (NHP) travels from the infected to the systemic leaf, where it induces the biosynthesis of salicylic acid (SA), resulting in a robust SAR response. Previous studies have shown that a subset of SAR related genes are induced even in the absence of SA biosynthesis, while gene induction is completely hampered in the absence of NHP biosynthesis. The purpose of this study was to decipher the SAindependent and NHP-dependent signalling cascade that activates gene expression during SAR. Using pharmacological treatment with NHP, we show that SAR-related genes such as FMO1, which encodes an enzyme involved in NHP biosynthesis, is induced even in the salicylic acid induction deficient 2 (sid2) mutant, which is devoid of pathogen-induced SA. The NHP-mediated induction of FMO1 required the SA receptor NON EXPRESSOR OF PATHOGENESIS-RELATED GENES1 (NPR1), and its interacting TGACG SEQUENCE-SPECIFIC BINDING PROTEIN (TGA) transcription factors. NHP treatment resulted in the accumulation of NPR1 protein in wild-type plants and to a lesser extent in the sid2 mutant. The structural similarity between SA and NHP prompted us to test if NHP binds to NPR1. Using isothermal titration calorimetry we show that while SA bound to NPR1 with a Kd value of $585 \pm 368 \mathrm{nM}$, no binding to NHP was detected. Moreover, we show that the nucleocytoplasmic protein ENHANCED DISEASE SUSCEPTIBILITY 1 (EDS1) was required for the NHP-induced expression of SAR-related genes. In this study, we show that NHP uses regulatory components of the SA signalling pathway to induce SAR genes, but the mechanism of its perception has remains an open question 


\section{1) Summary II - The glutaredoxin ROXY19 is a negative regulator of the JA/ET-dependent defense pathway}

Glutaredoxins (GRXs) are ubiquitous small proteins that function as thiolreductases or as scaffold proteins coordinating Fe-S clusters. Previous work from our lab has shown that the CC-type glutaredoxins ROXY18 and ROXY19 are inducible by salicylic acid (SA), interact with TGA2 transcription factor and that the overexpression of ROXY19 results in the repression of the jasmonic acid/ethylene (JA/ET)-induced expression of ORA59 and PDF1.2. This suggests that ROXY18 and ROXY19 are involved in the SA-mediated repression of the JA/ET pathway. The aim of the current work was to address the role of ROXY18/19 in the SA signalling cascade by utilizing the loss of function mutants generated by CRISPR-Cas-mediated gene editing. Gene expression analysis after Psm infection assays supported the notion that ROXY18 and 19 are involved in the SA-mediated repression of ORA59. Firstly, we observed that similar to the sid 2 mutant, the roxy18 roxy19 mutant showed high induction of ORA59 after Psm treatment. Prior data suggested that ROXY19 is recruited to the promoter site by TGA2 transcription factor, where it exerts its repressive effect. Supporting this model, our data show that the tga2 tga5 tga6 triple mutant shows hyperinduction of ORA59 after Psm infection, similar to the roxy mutants. The requirement of TGA2 for SA-mediated induction of ROXY19 however makes it difficult to pin down if the hyperinduction of ORA59 in tga2tga5tga6 is due to an impairment in the recruitment of ROXY19 to ORA59 promoter, or due to the reduced induction of ROXY19 in the tga mutant. Next, we observed that the downstream target of ORA59 - PDF1.2 is hyperinduced in mock-Psm and PsmPsm treated sid2 mutant, confirming the involvement of SA-mediated pathway in repressing PDF1.2. The induction of PDF1.2 was high in mock-Psm treated roxy mutants, but unlike the sid2 mutant, the Psm-Psm treated roxy mutants showed a 
repression of PDF1.2, implying that a ROXY-independent and SA-dependent pathway is involved in the repression of PDF1.2. In this work, we show loss of function evidence supporting the role of ROXY19 in SA-mediated repression of ORA59; further experiments using this system will provide more insights into the mechanistic aspects of ROXY19 mediated control of gene expression. 


\section{1) References}

Aarts, N., M. Metz, E. Holub, B.J. Staskawicz, M.J. Daniels, and J.E. Parker: Different requirements for EDS1 and NDR1 by disease resistance genes define at least two R gene-mediated signaling pathways in Arabidopsis. Proc Natl Acad Sci U S A 95, 10306-10311 (1998).

Abramovitch, R.B., Y.J. Kim, S. Chen, M.B. Dickman, and G.B. Martin: Pseudomonas type III effector AvrPtoB induces plant disease susceptibility by inhibition of host programmed cell death. EMBO J 22, 60-69 (2003).

Bandyopadhyay, S., F. Gama, M.M. Molina-Navarro, J.M. Gualberto, R. Claxton, S.G. Naik, B.H. Huynh, E. Herrero, J.P. Jacquot, M.K. Johnson, and N. Rouhier: Chloroplast monothiol glutaredoxins as scaffold proteins for the assembly and delivery of [2Fe-2S] clusters. Embo J 27, 1122-1133 (2008).

Bartsch, M., E. Gobbato, P. Bednarek, S. Debey, J.L. Schultze, J. Bautor, and J.E. Parker: Salicylic acid-independent ENHANCED DISEASE SUSCEPTIBILITY1 signaling in Arabidopsis immunity and cell death is regulated by the monooxygenase FMO1 and the Nudix hydrolase NUDT7. Plant Cell 18, 1038-1051 (2006).

Baum, S., E.M. Reimer-Michalski, A. Bolger, A.J. Mantai, V. Benes, B. Usadel, and U. Conrath: Isolation of Open Chromatin Identifies Regulators of Systemic Acquired Resistance. Plant Physiol 181, 817-833 (2019).

Bender, K.W., X. Wang, G.B. Cheng, H.S. Kim, R.E. Zielinski, and S.C. Huber: Glutaredoxin AtGRXC2 catalyses inhibitory glutathionylation of Arabidopsis BRI1-associated receptor-like kinase 1 (BAK1) in vitro. Biochem J 467, 399-413 (2015).

Bernsdorff, F., A.C. Doring, K. Gruner, S. Schuck, A. Brautigam, and J. Zeier: Pipecolic Acid Orchestrates Plant Systemic Acquired Resistance and Defense Priming via Salicylic Acid-Dependent and -Independent Pathways. Plant Cell 28, 102-129 (2016).

Bhandari, D.D., D. Lapin, B. Kracher, P. von Born, J. Bautor, K. Niefind, and J.E. Parker: An EDS1 heterodimer signalling surface enforces timely reprogramming of immunity genes in Arabidopsis. Nat Commun 10, 772 (2019).

Boller, T., and G. Felix: A renaissance of elicitors: perception of microbe-associated molecular patterns and danger signals by pattern-recognition receptors. Annu Rev Plant Biol 60, 379-406 (2009).

Bonardi, V., and J.L. Dangl: How complex are intracellular immune receptor signaling complexes? Front Plant Sci 3, 237 (2012).

Boudsocq, M., and J. Sheen: CDPKs in immune and stress signaling. Trends Plant Sci 18, 30-40 (2013).

Breitenbach, H.H., M. Wenig, F. Wittek, L. Jorda, A.M. Maldonado-Alconada, H. Sarioglu, T. Colby, C. Knappe, M. Bichlmeier, E. Pabst, D. Mackey, J.E. Parker, and A.C. Vlot: Contrasting Roles of the Apoplastic Aspartyl Protease APOPLASTIC, ENHANCED 
DISEASE SUSCEPTIBILITY1-DEPENDENT1 and LEGUME LECTIN-LIKE PROTEIN1 in Arabidopsis Systemic Acquired Resistance. Plant Physiol 165, 791-809 (2014).

Buttner, D.: Behind the lines-actions of bacterial type III effector proteins in plant cells. FEMS Microbiol Rev 40, 894-937 (2016).

Cao, H., S.A. Bowling, A.S. Gordon, and X. Dong: Characterization of an Arabidopsis Mutant That Is Nonresponsive to Inducers of Systemic Acquired Resistance. Plant Cell 6, 1583-1592 (1994).

Chaturvedi, R., B. Venables, R.A. Petros, V. Nalam, M. Li, X. Wang, L.J. Takemoto, and J. Shah: An abietane diterpenoid is a potent activator of systemic acquired resistance. Plant J 71, 161-172 (2012).

Chen, Y.C., E.C. Holmes, J. Rajniak, J.G. Kim, S. Tang, C.R. Fischer, M.B. Mudgett, and E.S. Sattely: N-hydroxy-pipecolic acid is a mobile metabolite that induces systemic disease resistance in Arabidopsis. Proc Natl Acad Sci U S A 115, E4920-E4929 (2018).

Chinchilla, D., Z. Bauer, M. Regenass, T. Boller, and G. Felix: The Arabidopsis receptor kinase FLS2 binds flg22 and determines the specificity of flagellin perception. Plant Cell 18, 465-476 (2006).

Chinchilla, D., C. Zipfel, S. Robatzek, B. Kemmerling, T. Nurnberger, J.D. Jones, G. Felix, and T. Boller: A flagellin-induced complex of the receptor FLS2 and BAK1 initiates plant defence. Nature 448, 497-500 (2007).

Collmer, A., J.L. Badel, A.O. Charkowski, W.L. Deng, D.E. Fouts, A.R. Ramos, A.H. Rehm, D.M. Anderson, O. Schneewind, K. van Dijk, and J.R. Alfano: Pseudomonas syringae Hrp type III secretion system and effector proteins. Proc Natl Acad Sci U S A 97, 8770-8777 (2000).

Conrath, U.: Systemic acquired resistance. Plant Signal Behav 1, 179-184 (2006).

Coppinger, P., P.P. Repetti, B. Day, D. Dahlbeck, A. Mehlert, and B.J. Staskawicz: Overexpression of the plasma membrane-localized NDR1 protein results in enhanced bacterial disease resistance in Arabidopsis thaliana. Plant J 40, 225237 (2004).

Corbett, E.F., K.M. Michalak, K. Oikawa, S. Johnson, I.D. Campbell, P. Eggleton, C. Kay, and M. Michalak: The conformation of calreticulin is influenced by the endoplasmic reticulum luminal environment. J Biol Chem 275, 27177-27185 (2000).

Couto, D., and C. Zipfel: Regulation of pattern recognition receptor signalling in plants. Nat Rev Immunol 16, 537-552 (2016).

Couturier, J., J.P. Jacquot, and N. Rouhier: Evolution and diversity of glutaredoxins in photosynthetic organisms. Cell Mol Life Sci 66, 2539-2557 (2009). 
Couturier, J., J.P. Jacquot, and N. Rouhier: Toward a refined classification of class I dithiol glutaredoxins from poplar: biochemical basis for the definition of two subclasses. Front Plant Sci 4, 518 (2013).

Delaney, T.P., L. Friedrich, and J.A. Ryals: Arabidopsis signal transduction mutant defective in chemically and biologically induced disease resistance. Proc Natl Acad Sci U S A 92, 6602-6606 (1995).

Dempsey, D.A., and D.F. Klessig: Salicylic acid, active oxygen species and systemic acquired resistance in plants. Trends Cell Biol 4, 334-338 (1994).

Deponte, M.: Glutathione catalysis and the reaction mechanisms of glutathionedependent enzymes. Biochim Biophys Acta 1830, 3217-3266 (2013).

Despres, C., C. Chubak, A. Rochon, R. Clark, T. Bethune, D. Desveaux, and P.R. Fobert: The Arabidopsis NPR1 disease resistance protein is a novel cofactor that confers redox regulation of DNA binding activity to the basic domain/leucine zipper transcription factor TGA1. Plant Cell 15, 2181-2191 (2003).

Ding, P., D. Rekhter, Y. Ding, K. Feussner, L. Busta, S. Haroth, S. Xu, X. Li, R. Jetter, I. Feussner, and Y. Zhang: Characterization of a Pipecolic Acid Biosynthesis Pathway Required for Systemic Acquired Resistance. Plant Cell 28, 2603-2615 (2016).

Ding, Y., T. Sun, K. Ao, Y. Peng, Y. Zhang, X. Li, and Y. Zhang: Opposite Roles of Salicylic Acid Receptors NPR1 and NPR3/NPR4 in Transcriptional Regulation of Plant Immunity. Cell 173, 1454-1467 e1415 (2018).

Dou, D., and J.M. Zhou: Phytopathogen effectors subverting host immunity: different foes, similar battleground. Cell Host Microbe 12, 484-495 (2012).

Durner, J., and D.F. Klessig: Salicylic acid is a modulator of tobacco and mammalian catalases. J Biol Chem 271, 28492-28501 (1996).

Durrant, W.E., and X. Dong: Systemic acquired resistance. Annu Rev Phytopathol 42, 185-209 (2004).

Falk, A., B.J. Feys, L.N. Frost, J.D. Jones, M.J. Daniels, and J.E. Parker: EDS1, an essential component of R gene-mediated disease resistance in Arabidopsis has homology to eukaryotic lipases. Proc Natl Acad Sci U S A 96, 3292-3297 (1999).

Felix, G., J.D. Duran, S. Volko, and T. Boller: Plants have a sensitive perception system for the most conserved domain of bacterial flagellin. Plant J 18, 265-276 (1999).

Feys, B.J., M. Wiermer, R.A. Bhat, L.J. Moisan, N. Medina-Escobar, C. Neu, A. Cabral, and J.E. Parker: Arabidopsis SENESCENCE-ASSOCIATED GENE101 stabilizes and signals within an ENHANCED DISEASE SUSCEPTIBILITY1 complex in plant innate immunity. Plant Cell 17, 2601-2613 (2005).

Fu, Z.Q., S. Yan, A. Saleh, W. Wang, J. Ruble, N. Oka, R. Mohan, S.H. Spoel, Y. Tada, N. Zheng, and X. Dong: NPR3 and NPR4 are receptors for the immune signal salicylic acid in plants. Nature 486, 228-232 (2012). 
Gaffney, T., L. Friedrich, B. Vernooij, D. Negrotto, G. Nye, S. Uknes, E. Ward, H. Kessmann, and J. Ryals: Requirement of salicylic Acid for the induction of systemic acquired resistance. Science 261, 754-756 (1993).

Gao, X., X. Chen, W. Lin, S. Chen, D. Lu, Y. Niu, L. Li, C. Cheng, M. McCormack, J. Sheen, L. Shan, and P. He: Bifurcation of Arabidopsis NLR immune signaling via $\mathrm{Ca}(2)(+)-$ dependent protein kinases. PLoS Pathog 9, e1003127 (2013).

Garcion, C., A. Lohmann, E. Lamodiere, J. Catinot, A. Buchala, P. Doermann, and J.P. Metraux: Characterization and biological function of the ISOCHORISMATE SYNTHASE2 gene of Arabidopsis. Plant Physiol 147, 1279-1287 (2008).

Groves, M.R., and D. Barford: Topological characteristics of helical repeat proteins. Curr Opin Struct Biol 9, 383-389 (1999).

Guo, B., H. Wang, B. Yang, W. Jiang, M. Jing, H. Li, Y. Xia, Y. Xu, Q. Hu, F. Wang, F. Yu, Y. Wang, W. Ye, S. Dong, W. Xing, and Y. Wang: Phytophthora sojae Effector PsAvh240 Inhibits Host Aspartic Protease Secretion to Promote Infection. Mol Plant 12, 552-564 (2019).

Hamiaux, C., R.S. Drummond, B.J. Janssen, S.E. Ledger, J.M. Cooney, R.D. Newcomb, and K.C. Snowden: DAD2 is an alpha/beta hydrolase likely to be involved in the perception of the plant branching hormone, strigolactone. Curr Biol 22, 20322036 (2012).

Han, S.W., and B.K. Hwang: Molecular functions of Xanthomonas type III effector AvrBsT and its plant interactors in cell death and defense signaling. Planta 245, 237-253 (2017).

Hartmann, M., D. Kim, F. Bernsdorff, Z. Ajami-Rashidi, N. Scholten, S. Schreiber, T. Zeier, S. Schuck, V. Reichel-Deland, and J. Zeier: Biochemical Principles and Functional Aspects of Pipecolic Acid Biosynthesis in Plant Immunity. Plant Physiol 174, 124-153 (2017).

Hartmann, M., T. Zeier, F. Bernsdorff, V. Reichel-Deland, D. Kim, M. Hohmann, N. Scholten, S. Schuck, A. Brautigam, T. Holzel, C. Ganter, and J. Zeier: Flavin Monooxygenase-Generated N-Hydroxypipecolic Acid Is a Critical Element of Plant Systemic Immunity. Cell 173, 456-469 e416 (2018).

Heath, M.C.: Nonhost resistance and nonspecific plant defenses. Curr Opin Plant Biol 3, 315-319 (2000).

Huang, L.J., N. Li, C. Thurow, M. Wirtz, R. Hell, and C. Gatz: Ectopically expressed glutaredoxin ROXY19 negatively regulates the detoxification pathway in Arabidopsis thaliana. BMC Plant Biol 16, 200 (2016).

Huang, W., Y. Wang, X. Li, and Y. Zhang: Biosynthesis and Regulation of Salicylic Acid and N-Hydroxypipecolic Acid in Plant Immunity. Mol Plant (2019).

Johnson, C., E. Boden, and J. Arias: Salicylic acid and NPR1 induce the recruitment of trans-activating TGA factors to a defense gene promoter in Arabidopsis. Plant Cell 15, 1846-1858 (2003). 
Jones, J.D., and J.L. Dangl: The plant immune system. Nature 444, 323-329 (2006).

Jung, H.W., T.J. Tschaplinski, L. Wang, J. Glazebrook, and J.T. Greenberg: Priming in systemic plant immunity. Science 324, 89-91 (2009).

Kim, C.Y., and S. Zhang: Activation of a mitogen-activated protein kinase cascade induces WRKY family of transcription factors and defense genes in tobacco. Plant J 38, 142-151 (2004).

Kim, M.C., W.S. Chung, D.J. Yun, and M.J. Cho: Calcium and calmodulin-mediated regulation of gene expression in plants. Mol Plant 2, 13-21 (2009).

Kim, Y., S.J. Gilmour, L. Chao, S. Park, and M.F. Thomashow: Arabidopsis CAMTA Transcription Factors Regulate Pipecolic Acid Biosynthesis and Priming of Immunity Genes. Mol Plant (2019).

Kim, Y., S. Park, S.J. Gilmour, and M.F. Thomashow: Roles of CAMTA transcription factors and salicylic acid in configuring the low-temperature transcriptome and freezing tolerance of Arabidopsis. Plant J 75, 364-376 (2013).

Kinkema, M., W. Fan, and X. Dong: Nuclear localization of NPR1 is required for activation of PR gene expression. Plant Cell 12, 2339-2350 (2000).

Knuesting, J., C. Riondet, C. Maria, I. Kruse, N. Becuwe, N. Konig, C. Berndt, S. Tourrette, J. Guilleminot-Montoya, E. Herrero, F. Gaymard, J. Balk, G. Belli, R. Scheibe, J.P. Reichheld, N. Rouhier, and P. Rey: Arabidopsis glutaredoxin S17 and its partner, the nuclear factor Y subunit C11/negative cofactor 2alpha, contribute to maintenance of the shoot apical meristem under long-day photoperiod. Plant Physiol 167, 1643-1658 (2015).

Kost, T.A., J.P. Condreay, and D.L. Jarvis: Baculovirus as versatile vectors for protein expression in insect and mammalian cells. Nat Biotechnol 23, 567-575 (2005).

Kuai, X., B.J. MacLeod, and C. Despres: Integrating data on the Arabidopsis NPR1/NPR3/NPR4 salicylic acid receptors; a differentiating argument. Front Plant Sci 6, 235 (2015).

La Camera, S., F. L'Haridon, J. Astier, M. Zander, E. Abou-Mansour, G. Page, C. Thurow, D. Wendehenne, C. Gatz, J.P. Metraux, and O. Lamotte: The glutaredoxin ATGRXS13 is required to facilitate Botrytis cinerea infection of Arabidopsis thaliana plants. Plant J 68, 507-519 (2011).

Lai, Y.S., L. Renna, J. Yarema, C. Ruberti, S.Y. He, and F. Brandizzi: Salicylic acidindependent role of NPR1 is required for protection from proteotoxic stress in the plant endoplasmic reticulum. Proc Natl Acad Sci U S A 115, E5203-E5212 (2018).

Laporte, D., E. Olate, P. Salinas, M. Salazar, X. Jordana, and L. Holuigue: Glutaredoxin GRXS13 plays a key role in protection against photooxidative stress in Arabidopsis. J Exp Bot 63, 503-515 (2011). 
Lebel, E., P. Heifetz, L. Thorne, S. Uknes, J. Ryals, and E. Ward: Functional analysis of regulatory sequences controlling $P R-1$ gene expression in Arabidopsis. Plant J 16, 223-233 (1998).

Li, S., A. Lauri, M. Ziemann, A. Busch, M. Bhave, and S. Zachgo: Nuclear activity of ROXY1, a glutaredoxin interacting with TGA factors, is required for petal development in Arabidopsis thaliana. Plant Cell 21, 429-441 (2009).

Lillig, C.H., C. Berndt, and A. Holmgren: Glutaredoxin systems. Biochim Biophys Acta 1780, 1304-1317 (2008).

Lindermayr, C., S. Sell, B. Muller, D. Leister, and J. Durner: Redox regulation of the NPR1-TGA1 system of Arabidopsis thaliana by nitric oxide. Plant Cell 22, 28942907 (2010).

Liu, P.P., C.C. von Dahl, and D.F. Klessig: The extent to which methyl salicylate is required for signaling systemic acquired resistance is dependent on exposure to light after infection. Plant Physiol 157, 2216-2226 (2011).

Lowy, P.H.: The conversion of lysine to pipecolic acid by Phaseolus vulgaris. Arch Biochem Biophys 47, 228-229 (1953).

Lu, D., S. Wu, X. Gao, Y. Zhang, L. Shan, and P. He: A receptor-like cytoplasmic kinase, BIK1, associates with a flagellin receptor complex to initiate plant innate immunity. Proc Natl Acad Sci U S A 107, 496-501 (2010).

Maekawa, T., T.A. Kufer, and P. Schulze-Lefert: NLR functions in plant and animal immune systems: so far and yet so close. Nat Immunol 12, 817-826 (2011).

Malamy, J., J.P. Carr, D.F. Klessig, and I. Raskin: Salicylic Acid: a likely endogenous signal in the resistance response of tobacco to viral infection. Science 250, 1002-1004 (1990).

Manohar, M., M. Tian, M. Moreau, S.W. Park, H.W. Choi, Z. Fei, G. Friso, M. Asif, P. Manosalva, C.C. von Dahl, K. Shi, S. Ma, S.P. Dinesh-Kumar, I. O'Doherty, F.C. Schroeder, K.J. van Wijk, and D.F. Klessig: Identification of multiple salicylic acid-binding proteins using two high throughput screens. Front Plant Sci 5, 777 (2014).

Mao, G., X. Meng, Y. Liu, Z. Zheng, Z. Chen, and S. Zhang: Phosphorylation of a WRKY transcription factor by two pathogen-responsive MAPKs drives phytoalexin biosynthesis in Arabidopsis. Plant Cell 23, 1639-1653 (2011).

Martin, J.L.: Thioredoxin--a fold for all reasons. Structure 3, 245-250 (1995).

Mine, A., C. Seyfferth, B. Kracher, M.L. Berens, D. Becker, and K. Tsuda: The Defense Phytohormone Signaling Network Enables Rapid, High-Amplitude Transcriptional Reprogramming during Effector-Triggered Immunity. Plant Cell 30, 1199-1219 (2018). 
Mishina, T.E., and J. Zeier: Pathogen-associated molecular pattern recognition rather than development of tissue necrosis contributes to bacterial induction of systemic acquired resistance in Arabidopsis. Plant J 50, 500-513 (2007).

Molders, W., A. Buchala, and J.P. Metraux: Transport of Salicylic Acid in Tobacco Necrosis Virus-Infected Cucumber Plants. Plant Physiol 112, 787-792 (1996).

Moseler, A., I. Aller, S. Wagner, T. Nietzel, J. Przybyla-Toscano, U. Muhlenhoff, R. Lill, C. Berndt, N. Rouhier, M. Schwarzlander, and A.J. Meyer: The mitochondrial monothiol glutaredoxin S15 is essential for iron-sulfur protein maturation in Arabidopsis thaliana. Proc Natl Acad Sci U S A 112, 13735-13740 (2015).

Mou, Z., W. Fan, and X. Dong: Inducers of plant systemic acquired resistance regulate NPR1 function through redox changes. Cell 113, 935-944 (2003).

Murmu, J., M.J. Bush, C. DeLong, S. Li, M. Xu, M. Khan, C. Malcolmson, P.R. Fobert, S. Zachgo, and S.R. Hepworth: Arabidopsis basic leucine-zipper transcription factors TGA9 and TGA10 interact with floral glutaredoxins ROXY1 and ROXY2 and are redundantly required for anther development. Plant Physiol 154, 14921504 (2010).

Navarova, H., F. Bernsdorff, A.C. Doring, and J. Zeier: Pipecolic acid, an endogenous mediator of defense amplification and priming, is a critical regulator of inducible plant immunity. Plant Cell 24, 5123-5141 (2012).

Nawrath, C., S. Heck, N. Parinthawong, and J.P. Metraux: EDS5, an essential component of salicylic acid-dependent signaling for disease resistance in Arabidopsis, is a member of the MATE transporter family. Plant Cell 14, 275-286 (2002).

Ndamukong, I., A.A. Abdallat, C. Thurow, B. Fode, M. Zander, R. Weigel, and C. Gatz: SAinducible Arabidopsis glutaredoxin interacts with TGA factors and suppresses JA-responsive PDF1.2 transcription. Plant J 50, 128-139 (2007).

Ohkubo, Y., M. Tanaka, R. Tabata, M. Ogawa-Ohnishi, and Y. Matsubayashi: Shoot-toroot mobile polypeptides involved in systemic regulation of nitrogen acquisition. Nature plants 3, 17029 (2017).

Piccinini, A.M., L. Zuliani-Alvarez, J.M. Lim, and K.S. Midwood: Distinct microenvironmental cues stimulate divergent TLR4-mediated signaling pathways in macrophages. Sci Signal 9, ra86 (2016).

Poovaiah, B.W., L. Du, H. Wang, and T. Yang: Recent advances in calcium/calmodulinmediated signaling with an emphasis on plant-microbe interactions. Plant Physiol 163, 531-542 (2013).

Pre, M., M. Atallah, A. Champion, M. De Vos, C.M. Pieterse, and J. Memelink: The AP2/ERF domain transcription factor ORA59 integrates jasmonic acid and ethylene signals in plant defense. Plant Physiol 147, 1347-1357 (2008).

Qi, Y., and N.V. Grishin: Structural classification of thioredoxin-like fold proteins. Proteins 58, 376-388 (2005). 
Rekhter, D., D. Ludke, Y. Ding, K. Feussner, K. Zienkiewicz, V. Lipka, M. Wiermer, Y. Zhang, and I. Feussner: Isochorismate-derived biosynthesis of the plant stress hormone salicylic acid. Science 365, 498-502 (2019).

Ribnicky, D.M., V.V. Shulaev, and I.I. Raskin: Intermediates of salicylic acid biosynthesis in tobacco. Plant Physiol 118, 565-572 (1998).

Richmond, T.A., and A.B. Bleecker: A defect in beta-oxidation causes abnormal inflorescence development in Arabidopsis. Plant Cell 11, 1911-1924 (1999).

Rietz, S., A. Stamm, S. Malonek, S. Wagner, D. Becker, N. Medina-Escobar, A.C. Vlot, B.J. Feys, K. Niefind, and J.E. Parker: Different roles of Enhanced Disease Susceptibility1 (EDS1) bound to and dissociated from Phytoalexin Deficient4 (PAD4) in Arabidopsis immunity. New Phytol 191, 107-119 (2011).

Riondet, C., J.P. Desouris, J.G. Montoya, Y. Chartier, Y. Meyer, and J.P. Reichheld: A dicotyledon-specific glutaredoxin GRXC1 family with dimer-dependent redox regulation is functionally redundant with GRXC2. Plant Cell Environ 35, 360373 (2012).

Ross, A.F: Systemic acquired resistance induced by localized virus infections in plants. Virology 14, 340-358 (1961)

Rothstein, M., and L.L. Miller: The conversion of lysine to pipecolic acid in the rat. J Biol Chem 211, 851-858 (1954).

Rouhier, N., C.S. Koh, E. Gelhaye, C. Corbier, F. Favier, C. Didierjean, and J.P. Jacquot: Redox based anti-oxidant systems in plants: biochemical and structural analyses. Biochim Biophys Acta 1780, 1249-1260 (2008a).

Rouhier, N., S.D. Lemaire, and J.P. Jacquot: The role of glutathione in photosynthetic organisms: emerging functions for glutaredoxins and glutathionylation. Annu Rev Plant Biol 59, 143-166 (2008b).

Rouhier, N., H. Unno, S. Bandyopadhyay, L. Masip, S.K. Kim, M. Hirasawa, J.M. Gualberto, V. Lattard, M. Kusunoki, D.B. Knaff, G. Georgiou, T. Hase, M.K. Johnson, and J.P. Jacquot: Functional, structural, and spectroscopic characterization of a glutathione-ligated [2Fe-2S] cluster in poplar glutaredoxin C1. Proc Natl Acad Sci U S A 104, 7379-7384 (2007).

Shearer, H.L., Y.T. Cheng, L. Wang, J. Liu, P. Boyle, C. Despres, Y. Zhang, X. Li, and P.R. Fobert: Arabidopsis clade I TGA transcription factors regulate plant defenses in an NPR1-independent fashion. Mol Plant Microbe Interact 25, 1459-1468 (2012).

Stroher, E., J. Grassl, C. Carrie, R. Fenske, J. Whelan, and A.H. Millar: Glutaredoxin S15 Is Involved in Fe-S Cluster Transfer in Mitochondria Influencing Lipoic AcidDependent Enzymes, Plant Growth, and Arsenic Tolerance in Arabidopsis. Plant Physiol 170, 1284-1299 (2016).

Sun, T., L. Busta, Q. Zhang, P. Ding, R. Jetter, and Y. Zhang: TGACG-BINDING FACTOR 1 (TGA1) and TGA4 regulate salicylic acid and pipecolic acid biosynthesis by 
modulating the expression of SYSTEMIC ACQUIRED RESISTANCE DEFICIENT 1 (SARD1) and CALMODULIN-BINDING PROTEIN 60g (CBP60g). New Phytol 217, 344-354 (2018).

Sun, T., J. Huang, Y. Xu, V. Verma, B. Jing, Y. Sun, A. Ruiz Orduna, H. Tian, X. Huang, S. Xia, L. Schafer, R. Jetter, Y. Zhang, and X. Li: Redundant CAMTA Transcription Factors Negatively Regulate the Biosynthesis of Salicylic Acid and NHydroxypipecolic Acid by Modulating the Expression of SARD1 and CBP60g. Mol Plant (2019).

Sun, T., Y. Zhang, Y. Li, Q. Zhang, Y. Ding, and Y. Zhang: ChIP-seq reveals broad roles of SARD1 and CBP60g in regulating plant immunity. Nat Commun 6, 10159 (2015).

Tada, Y., S.H. Spoel, K. Pajerowska-Mukhtar, Z. Mou, J. Song, C. Wang, J. Zuo, and X. Dong: Plant immunity requires conformational changes [corrected] of NPR1 via Snitrosylation and thioredoxins. Science 321, 952-956 (2008a).

Tada, Y., S.H. Spoel, K. Pajerowska-Mukhtar, Z. Mou, J. Song, C. Wang, J. Zuo, and X. Dong: Plant immunity requires conformational charges of NPR1 via S-nitrosylation and thioredoxins. Science 321, 952-956 (2008b).

Thordal-Christensen, H.: Fresh insights into processes of nonhost resistance. Curr Opin Plant Biol 6, 351-357 (2003).

Torrens-Spence, M.P., A. Bobokalonova, V. Carballo, C.M. Glinkerman, T. Pluskal, A. Shen, and J.K. Weng: PBS3 and EPS1 Complete Salicylic Acid Biosynthesis from Isochorismate in Arabidopsis. Mol Plant 12, 1577-1586 (2019).

Uhrig, J.F., L.J. Huang, S. Barghahn, M. Willmer, C. Thurow, and C. Gatz: CC-type glutaredoxins recruit the transcriptional co-repressor TOPLESS to TGAdependent target promoters in Arabidopsis thaliana. Biochim Biophys Acta Gene Regul Mech 1860, 218-226 (2017).

Uknes, S., B. Mauch-Mani, M. Moyer, S. Potter, S. Williams, S. Dincher, D. Chandler, A. Slusarenko, E. Ward, and J. Ryals: Acquired resistance in Arabidopsis. Plant Cell 4, 645-656 (1992).

Van de Weyer, A.L., F. Monteiro, O.J. Furzer, M.T. Nishimura, V. Cevik, K. Witek, J.D.G. Jones, J.L. Dangl, D. Weigel, and F. Bemm: A Species-Wide Inventory of NLR Genes and Alleles in Arabidopsis thaliana. Cell 178, 1260-1272 e1214 (2019).

Van der Does, D., A. Leon-Reyes, A. Koornneef, M.C. Van Verk, N. Rodenburg, L. Pauwels, A. Goossens, A.P. Korbes, J. Memelink, T. Ritsema, S.C. Van Wees, and C.M. Pieterse: Salicylic acid suppresses jasmonic acid signaling downstream of SCFCOI1-JAZ by targeting GCC promoter motifs via transcription factor ORA59. Plant Cell 25, 744-761 (2013).

van Verk, M.C., J.F. Bol, and H.J. Linthorst: WRKY transcription factors involved in activation of SA biosynthesis genes. BMC Plant Biol 11, 89 (2011). 
Vernooij, B., L. Friedrich, A. Morse, R. Reist, R. Kolditz-Jawhar, E. Ward, S. Uknes, H. Kessmann, and J. Ryals: Salicylic Acid Is Not the Translocated Signal Responsible for Inducing Systemic Acquired Resistance but Is Required in Signal Transduction. Plant Cell 6, 959-965 (1994a).

Vernooij, B., S. Uknes, E. Ward, and J. Ryals: Salicylic acid as a signal molecule in plantpathogen interactions. Curr Opin Cell Biol 6, 275-279 (1994b).

Vlot, A.C., D.A. Dempsey, and D.F. Klessig: Salicylic Acid, a multifaceted hormone to combat disease. Annu Rev Phytopathol 47, 177-206 (2009).

Wagner, S., J. Stuttmann, S. Rietz, R. Guerois, E. Brunstein, J. Bautor, K. Niefind, and J.E. Parker: Structural basis for signaling by exclusive EDS1 heteromeric complexes with SAG101 or PAD4 in plant innate immunity. Cell Host Microbe 14, 619-630 (2013).

Walters, L.A., and M.A. Escobar: The AtGRXS3/4/5/7/8 glutaredoxin gene cluster on Arabidopsis thaliana chromosome 4 is coordinately regulated by nitrate and appears to control primary root growth. Plant signaling \& behavior 11, e1171450 (2016).

Wang, L., K. Tsuda, M. Sato, J.D. Cohen, F. Katagiri, and J. Glazebrook: Arabidopsis CaM binding protein CBP60g contributes to MAMP-induced SA accumulation and is involved in disease resistance against Pseudomonas syringae. PLoS Pathog $\mathbf{5}$, e1000301 (2009).

Wang, X., J. Gao, Z. Zhu, X. Dong, X. Wang, G. Ren, X. Zhou, and B. Kuai: TCP transcription factors are critical for the coordinated regulation of isochorismate synthase 1 expression in Arabidopsis thaliana. Plant J 82, 151-162 (2015).

Wang, Y., and Y. Wang: Trick or Treat: Microbial Pathogens Evolved Apoplastic Effectors Modulating Plant Susceptibility to Infection. Mol Plant Microbe Interact 31, 6-12 (2018).

Wiermer, M., B.J. Feys, and J.E. Parker: Plant immunity: the EDS1 regulatory node. Curr Opin Plant Biol 8, 383-389 (2005).

Wildermuth, M.C., J. Dewdney, G. Wu, and F.M. Ausubel: Isochorismate synthase is required to synthesize salicylic acid for plant defence. Nature 414, 562-565 (2001).

Wittek, F., T. Hoffmann, B. Kanawati, M. Bichlmeier, C. Knappe, M. Wenig, P. SchmittKopplin, J.E. Parker, W. Schwab, and A.C. Vlot: Arabidopsis ENHANCED DISEASE SUSCEPTIBILITY1 promotes systemic acquired resistance via azelaic acid and its precursor 9-oxo nonanoic acid. J Exp Bot 65, 5919-5931 (2014).

Wu, Y., D. Zhang, J.Y. Chu, P. Boyle, Y. Wang, I.D. Brindle, V. De Luca, and C. Despres: The Arabidopsis NPR1 protein is a receptor for the plant defense hormone salicylic acid. Cell Rep 1, 639-647 (2012).

Xing, S., A. Lauri, and S. Zachgo: Redox regulation and flower development: a novel function for glutaredoxins. Plant Biol (Stuttg) 8, 547-555 (2006). 
Xing, S., M.G. Rosso, and S. Zachgo: ROXY1, a member of the plant glutaredoxin family, is required for petal development in Arabidopsis thaliana. Development 132, 1555-1565 (2005).

Xing, S., and S. Zachgo: ROXY1 and ROXY2, two Arabidopsis glutaredoxin genes, are required for anther development. Plant J 53, 790-801 (2008).

Xiong, Y., C. DeFraia, D. Williams, X. Zhang, and Z. Mou: Characterization of Arabidopsis 6-phosphogluconolactonase T-DNA insertion mutants reveals an essential role for the oxidative section of the plastidic pentose phosphate pathway in plant growth and development. Plant Cell Physiol 50, 1277-1291 (2009).

Zander, M., S. Chen, J. Imkampe, C. Thurow, and C. Gatz: Repression of the Arabidopsis thaliana jasmonic acid/ethylene-induced defense pathway by TGA-interacting glutaredoxins depends on their C-terminal ALWL motif. Mol Plant 5, 831-840 (2012).

Zander, M., C. Thurow, and C. Gatz: TGA Transcription Factors Activate the Salicylic Acid-Suppressible Branch of the Ethylene-Induced Defense Program by Regulating ORA59 Expression. Plant Physiol 165, 1671-1683 (2014).

Zhang, L., L. Du, C. Shen, Y. Yang, and B.W. Poovaiah: Regulation of plant immunity through ubiquitin-mediated modulation of $\mathrm{Ca}(2+)$-calmodulin-AtSR1/CAMTA3 signaling. Plant J 78, 269-281 (2014).

Zhang, Y., W. Fan, M. Kinkema, X. Li, and X. Dong: Interaction of NPR1 with basic leucine zipper protein transcription factors that bind sequences required for salicylic acid induction of the PR-1 gene. Proc Natl Acad Sci U S A 96, 6523-6528 (1999).

Zhang, Y., M.J. Tessaro, M. Lassner, and X. Li: Knockout analysis of Arabidopsis transcription factors TGA2, TGA5, and TGA6 reveals their redundant and essential roles in systemic acquired resistance. Plant Cell 15, 2647-2653 (2003).

Zhang, Y., L. Zhao, J. Zhao, Y. Li, J. Wang, R. Guo, S. Gan, C.J. Liu, and K. Zhang: S5H/DMR6 Encodes a Salicylic Acid 5-Hydroxylase That Fine-Tunes Salicylic Acid Homeostasis. Plant Physiol 175, 1082-1093 (2017).

Zheng, X.Y., N.W. Spivey, W. Zeng, P.P. Liu, Z.Q. Fu, D.F. Klessig, S.Y. He, and X. Dong: Coronatine promotes Pseudomonas syringae virulence in plants by activating a signaling cascade that inhibits salicylic acid accumulation. Cell Host Microbe 11, 587-596 (2012).

Zhou, J.M., Y. Trifa, H. Silva, D. Pontier, E. Lam, J. Shah, and D.F. Klessig: NPR1 differentially interacts with members of the TGA/OBF family of transcription factors that bind an element of the PR-1 gene required for induction by salicylic acid. Mol Plant Microbe Interact 13, 191-202 (2000).

Ziemann, M., M. Bhave, and S. Zachgo: Origin and diversification of land plant CC-type glutaredoxins. Genome Biol Evol 1, 265-277 (2009). 


\section{2) Abbreviations}

\begin{tabular}{|c|c|}
\hline Abbreviation & Extended spelling \\
\hline A. thaliana & Arabidopsis thaliana \\
\hline AcMNPV & $\begin{array}{l}\text { Autographa californica multinucleocapsid } \\
\text { nucleopolyhedrovirus }\end{array}$ \\
\hline AIM1 & ABNORMAL INFLORESCENCE MERISTEM 1 \\
\hline ALD1 & AGD2-LIKE DEFENSE RESPONSE PROTEIN 1 \\
\hline $\mathrm{Amp}^{\mathrm{R}}$ & Ampicillin resistance \\
\hline ANOVA & Analysis of Variance \\
\hline APS & Ammonium peroxodisulfate \\
\hline ATP & Adenosinetriphosphate \\
\hline BAK1 & $\begin{array}{l}\text { BRASSINOSTEROID INSENSITIVE 1-ASSOCIATED } \\
\text { RECEPTOR KINASE } 1\end{array}$ \\
\hline BGL2 & $\beta$-1,3-GLUCANASE \\
\hline BIK1 & BOTRYTIS-INDUCED KINASE 1 \\
\hline C, Cys & Cysteine \\
\hline CBP60G & CALMODULIN BINDING PROTEIN 60G \\
\hline cDNA & complementary DNA \\
\hline dATP & deoxy Adenosine Triphosphate \\
\hline dCTP & deoxy Cytidine Triphosphate \\
\hline dGTP & deoxy Guanosine Triphosphate \\
\hline DNA & Deoxyribonucleic Acid \\
\hline
\end{tabular}




\begin{tabular}{|c|c|}
\hline DNA & Deoxyribonucleic acid \\
\hline dNTPs & Deoxyribonucteotide Triphosphate \\
\hline DTT & Dithiothreitol \\
\hline dTTP & deoxy Tymidine Triphosphate \\
\hline dYT & Yeast extract and Tryptone media \\
\hline E.coli & Escherichia coli \\
\hline EDS1 & ENHANCED SYSCEPTIBILITY 1 \\
\hline EDS5 & ENHANCED DISEASE SUSCEPTIBILITY 5 \\
\hline EDTA & Ethylene Diamine Tetra-acetic Acid \\
\hline EGF & Epidermal Growth Factor \\
\hline EPS1 & ENHANCED PSEUDOMONAS SUSCEPTIBILITY 1 \\
\hline Escherichia coli & E. coli \\
\hline ET & ethylene \\
\hline ETS & Effector Triggered Susceptibility \\
\hline FLS2 & FLAGELLIN SENSING 2 \\
\hline FM01 & FLAVIN MONOOXYGENASE 1 \\
\hline GRXs & glutaredoxins \\
\hline HR & Hypersensitive Response \\
\hline IC & Isochorismate \\
\hline ICS1 & ISOCHORISMATE SYNTHASE 1 \\
\hline INA & 2,6-dichloroisonicotinic acid \\
\hline ITC & Isothermal titration calorimetry \\
\hline$J A$ & Jasmonic acid \\
\hline $\operatorname{Kan}^{\mathrm{R}}$ & Kanamycin resistance \\
\hline
\end{tabular}

\section{Abbreviation $\quad$ Extended spelling}




\begin{tabular}{|c|c|}
\hline LRR & Leucine-rich repeat \\
\hline LysM & Lysine motifs \\
\hline MAPK & Mitogen Associated Protein Kinase \\
\hline NADPH & $\begin{array}{l}\text { Nicotinamide adenine dinucleotide phosphate, reduced } \\
\text { form }\end{array}$ \\
\hline NDR1 & NON-RACE-SPECIFIC-DISEASE RESISTANCE 1 \\
\hline NHP & N-Hydroxypipecolic acid \\
\hline NPR1 & NON EXPRESSOR OF PATHOGENESIS-RELATED GENES 1 \\
\hline O.D. & Optical density \\
\hline ORA59 & $\begin{array}{l}\text { OCTADECANOID-RESPONSIVE ARABIDOPSIS AP2/ERF } \\
\text { DOMAIN PROTEIN } 59\end{array}$ \\
\hline$p$ & $p$-value \\
\hline PAD4 & PHYTOALEXIN DEFICIENT 4 \\
\hline PAL & PHENYLALANINE AMMONIA-LYASE \\
\hline PAMPs/MAMPs & Pathogen/Microbe Associated Molecular Patterns \\
\hline PBS3 & AVRPPHB SUSCEPTIBLE 3 \\
\hline PCR & Polymerase Chain Reaction \\
\hline PDF1.2 & PLANT DEFENSIN 1.2 \\
\hline PR1 & PATHOGENESIS-RELATED GENE 1 \\
\hline
\end{tabular}

\section{Abbreviation} Extended spelling

PRRs

pattern recognition receptors 


$\begin{array}{ll}\text { Psm } & \text { Pseudomonas syringae pv. maculicola } \\ \text { PTI } & \text { PAMP-Triggered Immunity } \\ \text { qRT-PCR } & \text { Quantitative Real-Time PCR } \\ \text { R-gene } & \text { RESISTANCE gene } \\ \text { RLKs } & \text { Receptor-Like Kinases } \\ \text { ROS } & \text { Reactive Oxygen Species } \\ \text { SA } & \text { Salicylic acid } \\ \text { SAG101 } & \text { SENESCENCE ASSOCIATED GENE 101 } \\ \text { SAR } & \text { Systemic acquired resistance } \\ \text { SARD1 } & \text { SAR DEFICIENT 1 } \\ \text { SARD4 } & \text { SAR DEFICIENT 4 } \\ \text { SDS-PAGE } & \text { SDS-Polyacrylamide Gel Electrophoresis } \\ \text { SnRK2.8 } & \text { SNF-RELATED PROTEIN KINASE 2.8 } \\ \text { TCEP } & \text { Tris(2-carboxyethyl)phosphine } \\ \text { T-DNA } & \text { Transfer DNA } \\ \text { TEMED } & \text { Tetramethylethylenediamine } \\ \text { TPL } & \text { TGAC SEQUENCE-SPECIFIC BINDING PROTEIN } \\ & \end{array}$

\begin{tabular}{ll}
\hline Abbreviation & Extended spelling \\
\hline TRX & THIOREDOXIN \\
UBQ & UBIQUITIN \\
\hline
\end{tabular}




$\begin{array}{ll}\text { YEB } & \text { Yeast Extract Broth media } \\ \alpha & \text { Alpha, antibody } \\ \beta & \text { Beta }\end{array}$

\begin{tabular}{ll}
\hline Unit sign & Extended spelling \\
$\%$ & per cent \\
${ }^{\circ} \mathrm{C}$ & degree Celsius \\
$\mathrm{L}, \mathrm{l}$ & Litre \\
$\mathrm{M}$ & Molar (mol/l) \\
$\mathrm{m}$ & Metre \\
$\mathrm{min}$ & Minutes \\
$\mathrm{mol}$ & Mol \\
$\mathrm{rpm}$ & rotations per minute \\
$\mathrm{S}, \mathrm{sec}$ & Seconds \\
$\mathrm{kDa}$ & Kilodalton \\
$\mathrm{Kd}$ & Dissociation constant \\
$\mu$ & micro (10-6)
\end{tabular}




\section{3) Supplementary figures}

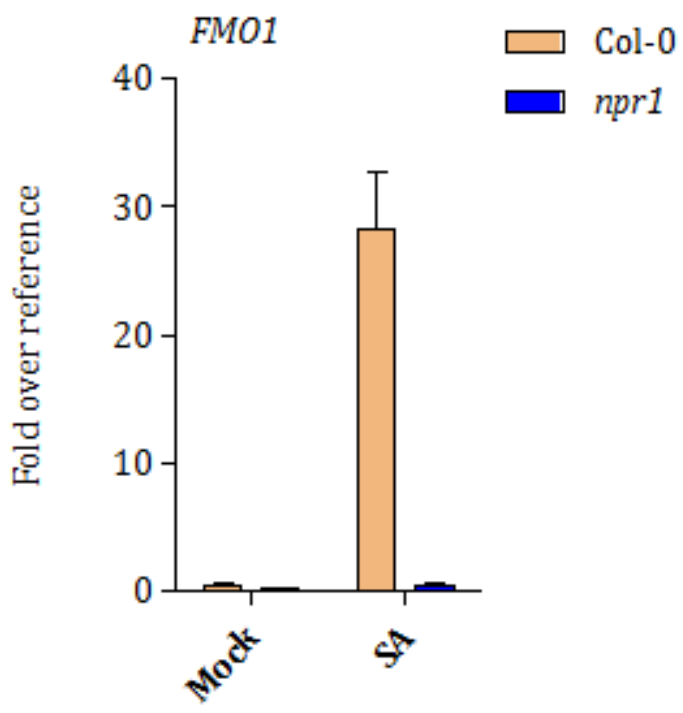

Figure 1: q-RT PCR analysis of FMO1 in Col-0 and npr1 plants following mock and SA treatment. Four and a half week old plants were sprayed with water or $1 \mathrm{mM}$ SA. The leaves were collected after 8 hours. Transcript levels were normalized to the transcript levels of $U B Q 5$. Error bars represent the average \pm SEM of five plants of each genotype 


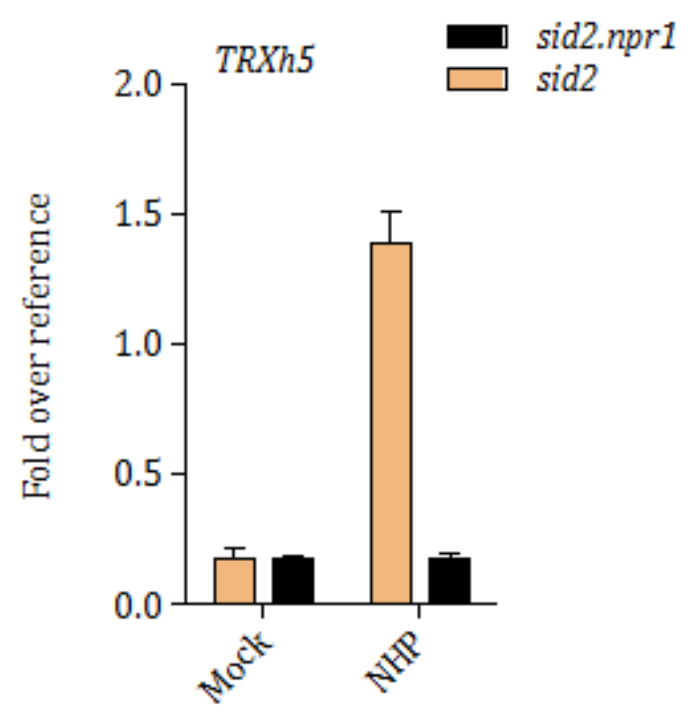

Figure 2: q-RT PCR analysis of TRXh5 in sid2, and sid2 npr1 plants following mock and NHP treatment. Three leaves of four and a half week old plants were infiltrated with $\mathrm{MgCl}_{2}$ or $1 \mathrm{mM} \mathrm{NHP.} \mathrm{The} \mathrm{leaves} \mathrm{were} \mathrm{collected} \mathrm{after} 48$ hours. Transcript levels were normalized to the transcript levels of UBQ5. Error bars represent the average \pm SEM of five plants of each genotype. 


\section{4) Acknowledgements}

They say it takes an entire village to raise a child. Similarly, it takes an entire department to raise a PhD student. I would like to thank everyone who has contributed to my "scientific" development.

Firstly, I would like to thank the boss (Prof. Gatz), for guiding me throughout without losing her cool, for the discussions and for targeting all the resources into the development of my thesis. Had I accepted some of her suggestions at an earlier stage of my PhD thesis, I would have most likely achieved a great deal more.

I would like to thank Prof. Feussner and Dr.Zhang for their guidance and for being the voices of reassurance during the most critical of times. I would like to thank Corinna, whose work ethics is incomparable and worthy of being followed, I would also like to thank her for her contributions in the design of my experiments. Lab meetings were often confusing and frustrating; I would like to thank Joachim for being kind enough to attend each one of my meetings, contributing in the discussions and being a calm presence. Most importantly, I would like to thank him for cloning the NPR1 constructs. I would like to thank Irene, for the discussions regarding my ROXY project and for letting me borrow her reagents. Ronny, for constantly being around and trying to lift my mood with his hilarious (not) jokes every time he sensed that my morale was down, which unfortunately was a very frequent event. I would like to thank Anna for her contribution in the generation of the roxy mutants, and for being around to clear my doubts. Ever so often, the RNA extraction work, which I found weirdly meditative, got annoyingly frustrating; I would like to thank Kathi for taking over during such situations. I would like to thank Guido for patiently answering the most trivial questions and for being around to prevent all the technical mishaps that I could have caused.

I would like to thank Jelena, Louisa, Daniel and their significant others Josh, Ali and Christina respectively, for being supportive, for calling me out on my nonsensical ideas and most importantly - for being an amazing group of peopleyou guys are the best!. I would like to thank Pascal and Isha for their contribution to my thesis. Pascal, for his work in the insect cell expression system and for being around to answer any of my protein-purification related doubts. Isha, for taking the time to run my protein samples. I would like to thank Lisa and Anja for being around and livening up the lab. 
Most importantly, I would like to thank my family - Acha, Amma and Ammu - for all their sacrifices and for believing in me. 